\title{
Multiomics Analyses Reveal Dynamic Bioenergetic Pathways and Functional Remodeling of the Heart During Intermittent Fasting
}

Thiruma V. Arumugam ${ }^{1,2,3, \#, * \text {, Asfa Alli-Shaik }}{ }^{4, \#}$, Elisa A. Liehn ${ }^{5,6,7}$, Sharmelee Selvaraji ${ }^{1,8}$, Luting Poh ${ }^{1}$, Vismitha Rajeev ${ }^{1}$, Joonki Kim ${ }^{1,9}$, Hannah L. F. Swa ${ }^{4}$, David Tan Zhi Hao ${ }^{1}$, Chutima Ratttanasopa ${ }^{10,11}$, David Yang-Wei Fann ${ }^{1}$, David Castano Mayan ${ }^{10}$, Gavin YongQuan $\mathrm{Ng}^{1}$, Sang-Ha Baik ${ }^{1}$, Karthik Mallilankaraman ${ }^{1}$, Mathias Gelderblom ${ }^{12}$, Grant R. Drummond $^{3}$, Christopher G. Sobey ${ }^{3}$, Brian K. Kennedy ${ }^{1,13,14}$, Dong-Gyu Jo ${ }^{2}$, Roshni R. Singaraja $^{10,15}$, Mark P. Mattson ${ }^{16}$, Jayantha Gunaratne $e^{4,17, *}$

${ }^{1}$ Department of Physiology, Yong Loo Lin School Medicine, National University of Singapore, Singapore

${ }^{2}$ School of Pharmacy, Sungkyunkwan University, Suwon, Republic of Korea

${ }^{3}$ Department of Physiology, Anatomy \& Microbiology, School of Life Sciences, La Trobe University, Bundoora, Victoria, Australia

${ }^{4}$ Translational Biomedical Proteomics Laboratory, Institute of Molecular and Cell Biology, Agency for Science, Technology and Research, Singapore

${ }^{5}$ National Heart Research Institute, National Heart Centre Singapore

${ }^{6}$ Institute of Molecular Cardiovascular Research, RWTH, Aachen, Germany

${ }^{7}$ Human Genetic Laboratory, University of Medicine, Craiova, Romania

${ }^{8}$ NUS Graduate School for Integrative Sciences and Engineering, National University of Singapore, Singapore

${ }^{9}$ Natural Products Research Center, Korea Institute of Science and Technology, Gangneung, Gangwon-do, Republic of Korea

${ }^{10}$ Translational Laboratories in Genetic Medicine, Agency for Science, Technology and Research, Singapore

${ }^{11}$ Cardiovascular and Metabolic Disorders Program, Duke-National University of Singapore

${ }^{12}$ Department of Neurology, University Medical Center Hamburg-Eppendorf, Germany

${ }^{13}$ Department of Biochemistry, Yong Loo Lin School Medicine, National University of Singapore, Singapore

${ }^{14}$ Buck Institute for Research on Aging, Novato, USA

${ }^{15}$ Department of Medicine, Yong Loo Lin School of Medicine, National University of Singapore, Singapore

${ }^{16}$ Department of Neuroscience, Johns Hopkins University School of Medicine, Baltimore, MD, USA

${ }^{17}$ Department of Anatomy, Yong Loo Lin School of Medicine, National University of Singapore, Singapore

Correspondence: Jayantha Gunaratne, Institute of Molecular and Cell Biology, Agency for Science, Technology and Research (A*STAR), Singapore, Singapore. E-mail: jayanthag@imcb.a-star.edu.sg or Thiruma V. Arumugam, Department of Physiology, Anatomy \& Microbiology, School of Life Sciences, La Trobe University, Bundoora, Victoria, Australia. E-mail: g.arumugam@latrobe.edu.au

\#These authors contributed equally to this work 


\section{Key words}

Intermittent fasting, heart, mouse, RNA sequencing, proteomics, phosphoproteomics, metabolism, echocardiogram

\section{SUMMARY}

Intermittent fasting (IF) reduces cardiovascular risk factors in animals and humans, and can protect the heart against ischemic injury in models of myocardial infarction, but the underlying molecular mechanisms are unknown. To delineate molecular and cellular adaptations of the heart to IF, we carried out comprehensive analyses of molecular pathways and biological processes using proteomic and phosphoproteomic data followed by functional analysis of hearts from mice maintained for 6 months on either daily 12- or 16-hour fasting, every-other-day fasting or ad libitum control feeding regimens. IF regimens significantly affected pathways that regulate cyclic GMP signaling, lipid and amino acid metabolism, cell adhesion, cell death and inflammation. Comparison of differentially expressed proteome and transcriptome upon IF showed higher correlation of pathway alternation in short IF regimen, but inverse correlation of metabolic processes such as fatty acid oxidation and immune processes in longer IF regimens. Echocardiographic analyses demonstrated that all three IF regimens enhance stress-induced cardiac performance. In addition to providing a valuable resource, our systemic analyses reveal molecular framework for understanding how IF impacts the function of the heart and its vulnerability to injury and disease.

\section{INTRODUCTION}

In the developed world, the average calorie intake has steadily risen as have associated agerelated diseases (Lahey and Khan, 2018). Intermittent fasting (IF) eating patterns include frequent periods of 12 to 24 hours with little or no energy intake sufficient to trigger a metabolic switch from utilization of liver-derived glucose to fatty acids and ketones. Such IF eating patterns increase health span and lifespan, and delay or prevent age-associated diseases in animals (de Cabo and Mattson, 2019; Mattson et al., 2017). Rodents maintained on IF exhibit reduced resting heart rate and blood pressure, increased heart rate variability and improved cardiovascular adaptations to stress (Mager et al., 2006; Wan et al., 2003a; Wan et al., 2003b; Wan et al., 2014). Age-related increases in oxidative stress, inflammation and fibrosis in the heart are prevented by every-other-day (EOD) fasting (Castello et al., 2011). IF 
also improves health indicators and cardiovascular risk factors in human subjects (Harvie et al., 2011; Harvie et al., 2013; Stein et al., 2012; Weiss and Fontana, 2011). In a recent randomized controlled trial, alternate day fasting improved cardiovascular markers, reduced fat mass, fat-to-lean ratio, and increased levels of the ketone $\beta$-hydroxybutyrate (BHB) (Stekovic et al., 2019). IF protects the brain and heart against ischemic injury and enhances endurance in animal models (Arumugam et al., 2010; Mattson and Arumugam 2018; Kim et al., 2018; Marosi et al., 2018; Wan et al., 2010). Animals maintained on EOD fasting prior to experimental myocardial infarction (MI) have a reduced myocardial infarct size compared to the ad libitum (AL) fed controls (Ahmet et al., 2005; Ahmet et al., 2011; Godar et al., 2015). IF also improves survival and recovery of heart function when initiated two weeks after MI (Katare et al., 2009).

Recent studies of the brain and skeletal muscle suggest that IF engages signaling pathways that bolster cellular stress resistance, mitochondrial biogenesis and autophagy (Marosi et al., 2018; Mattson and Arumugam 2018). The ketone BHB may mediate some cellular adaptations to fasting including inducing the expression of the inhibitory neurotransmitter $\gamma$ aminobutyric acid, trophic factors and the cytoprotective mitochondrial deacetylase sirtuin 3 (Cheng et al., 2016; Marosi et al., 2016; Newman and Verdin, 2017; Liu et al., 2019; Yudkoff et al., 2007). It is not known whether similar molecular and cellular mechanisms underlie the beneficial effects of IF on the cardiovascular system.

In order to understand molecular and cellular remodeling of the heart during IF, we employed advanced mass spectrometry for system-wide profiling of the proteome and phosphoproteome of heart tissues obtained from mice with daily fasting for 12 (IF12), 16 hours (IF16) or EOD as well as AL control feeding. We also performed transcriptome analyses using RNA sequencing to evaluate whether the observed molecular responses to IF occur at the transcriptional or post-transcriptional levels. Our results indicated that IF profoundly modifies pathways involved in metabolism, cell signaling and epigenetic reprograming in heart. Of note, we observed that protein network remodeling during IF occurred in an IF duration timedependent manner with IF16 and EOD having the greatest effects. In addition, functional studies indicated that IF improves cardiac function compared to AL feeding. Our findings provide novel insight into the genetic and proteomic changes by which IF improves cardiac health and provide a resource for investigators in the fields of metabolism and cardiovascular disease. 


\section{RESULTS}

Male C57BL/6N mice were fed a normal chow diet comprised on a caloric basis of 58\%, 24\% and $18 \%$ carbohydrate, protein and fat, respectively. Mice were assigned randomly to either AL, daily IF12, IF16 or EOD schedules beginning at 3 months of age. The study design, including the timing of experimental interventions, and blood and tissue collections, is summarized in Figure 1. To determine the extent to which IF affects energy metabolism, we measured body weight, and blood glucose and ketone levels of all mice during the six-month dietary intervention period. Mice in the AL group exhibited a trend towards increased blood glucose levels at 3 and 6-months. In contrast, mice in the EOD group exhibited a large and highly significant decrease in blood glucose levels throughout the duration of the study (Fig. 1). Mice in the IF12 and IF16 groups exhibited glucose levels intermediate to the AL and EOD groups (Fig. 1). Ketone levels also displayed variability across different fasting regimens. On fasting days mice in the EOD group exhibited blood ketone levels that were 3fold greater than mice in the AL group. Ketone levels in mice in the IF16 and IF12 groups tended to be higher than those in the AL group, but were substantially lower than the EOD group. Notably, all IF and EOD animals exhibited significantly lower body weight compared to AL mice. However, we did not observe any difference in overall body weight between the IF and EOD groups despite the differences in the fasting regimen pattern (Supplementary Fig. 1).

\section{IF modifies the heart proteome}

To investigate the impact of IF on the heart, we carried out comprehensive proteome profiling of at least four biological replicates from each dietary group using tandem mass tag (TMT)based high-resolution proteomics mass spectrometry. We identified a total of 6,972 proteins, of which 524 proteins were differentially expressed (ANOVA, FDR < 0.1) among the heart tissues from different groups (Tables S1 and S2). Of all proteins that displayed groupspecific protein abundance changes in comparison with AL (post hoc Tukey test, p value < 0.05), only 94 consistently displayed altered protein levels with IF across all fasting regimens (Supplementary Fig. 2). Among these, the majority were reduced in abundance and primarily included proteins involved in glycogen catabolism, fatty acid oxidation and glycolysis, suggesting that IF metabolically reprograms the heart. The EOD group exhibited the greatest number of protein abundance changes with $34 \%$ modulations exclusively occurring within this group, including apolipoproteins that are important determinants of a 
healthy heart (Liehn et al., 2018). Of note, more than one-third of the differentially expressed proteins in IF groups compared to AL were enzymes with catalytic activity, including those that regulate energy metabolism (Supplementary Fig. 3). Most of the enzymes involved in glycolysis including the rate-limiting enzyme phosphofructokinase (PFKFKB1/2) displayed decreased expression with IF except for hexokinase 1 (HK1), indicative of suppression of glycolysis (Table S2). Conversely, expression of phosphoenolpyruvate carboxykinase (PCK2), which is involved in gluconeogenesis, was enhanced by IF. We also observed distinct expression modulation of several kinases and phosphatases with IF (Supplementary Fig. 3). Critical determinants of insulin response and signaling including AKT1 and IGF1R showed a specific increase in expression in the EOD group (Table S2).

Unsupervised hierarchical clustering of the altered heart proteome revealed that IF12, IF16 and EOD mice had different overall protein expression patterns and co-expressed protein clusters compared to mice in the AL group (Fig. 2a). Unlike clusters in IF16 and IF12 groups, which exhibited modest differences compared to the AL group, a distinct class of proteins showed increased abundance only in the EOD group. Enrichment of specific biological processes and pathways (FDR < 0.05) showed that many of the altered clusters underlie key functional signatures. For example, the cluster with exclusive upregulation of proteins in the EOD group showed enrichment for gene expression and RNA-related processes such as RNA processing and splicing (Fig. 2b), highlighting that molecular remodeling in heart cells accompanies a longer period of fasting (EOD) compared to shorter daily fasts. Several transporters, receptors or ribosyltransferases (annotated as 'integral to membrane') showed concomitant upregulation in all IF groups compared to the AL group (Fig. 2b). Conversely, we observed that protein clusters associated with catabolic processes are reduced in all IF groups compared to AL. Expression of proteins involved in cholesterol and triglyceride metabolism/transport and many mitochondrial enzymes also exhibited a decline with IF.

To understand the molecular events accompanying each IF regimen, we performed 1-D annotation enrichment (Cox and Mann, 2012) that identifies processes enriched at higher and lower protein abundances (Fig. 2c and Table S3). We observed upregulation of focal adhesion, cell adhesion and extracellular matrix (ECM) receptor interaction proteins consistently with IF across all fasting regimens, suggesting that IF structurally remodels the heart. In addition, proteins regulating lipid kinase activity were observed in higher abundance in the hearts of mice from all three IF regimens compared to AL. We also observed certain 
signaling pathways to be selectively enriched in one IF regimen compared to the other IF regimens. For example, the IF12 group showed enrichment of proteins associated with cyclase activity while the IF16 group displayed elevation of ERK signaling proteins. Signaling pathways involving the second messenger cAMP, however, were activated only with longer periods of fasting. This supports the notion that different IF regimens differentially modulate protein expression changes in the heart and impact signaling rewiring (Fig. 2c and Table S3). Apart from components of lipid metabolism and transport that were reduced across all IF groups, our data also showed that branched-chain amino acid (BCAA) metabolism, which are known to regulate several anabolic and catabolic cellular cascades (Gannon et al., 2018), was significantly lower in all IF groups compared to the AL group (Fig. 2c).

\section{Post-transcriptional buffering of IF-responsive genes}

The major scope of this study was to understand IF-modulated molecular mechanisms through expression patterns of proteins since they are the ultimate functional effectors that are both reflective of, and more directly correlated with phenotypes. It has been well documented that post-transcriptional regulation can result in discordant levels of mRNA and protein levels primarily via translational/post-translational control or protein degradation (Jagannathan et al., 2019; Liu et al., 2016). Hence, we next compared the proteome and transcriptome to assess the level of correlation across the different groups and to unravel any deviations in a particular dietary regimen or even for specific sets of proteins within a regimen. For this, we performed RNA-seq based transcriptome profiling for heart tissues obtained from mice in different IF regimens (Supplementary Fig. 4 and Table S4). The transcriptome landscape revealed distinct patterns of gene expression across the different IF regimens with differentially modulated genes involved in various metabolic processes. For approximately 93\% of the proteins that were quantified across the different regimens, we obtained corresponding transcript readouts using RNA sequencing. Overall abundance correlation between the transcriptome and proteome revealed only a modest correlation, as expected (Pearson correlation coefficient 0.47) (Fig. 3a). However, comparisons of the IF-induced alterations at the proteome and transcriptome level showed rather poor correlation (Supplementary Fig. 5).

To further investigate this, we performed 2-D enrichment analyses to reveal correlated and non-correlated functional processes at the proteome and transcriptome levels (Table S5). This 
revealed that structural processes such as ECM-receptor interaction, and metabolic pathways involving BCAA degradation showed consistent changes in proteome and transcriptome with IF (Fig. 3b). Nevertheless, with increased fasting hours, mainly in the EOD group, we observed a trend shift in the direction of proteome and transcriptome abundances (Fig. 3b). Metabolic processes associated with fatty acid oxidation and monocarboxylic acid catabolic processes, and immune processes related to autoimmunity and chemokine signaling showed most obvious shifts. On investigating the fatty acid oxidation cluster, we noticed that many of the key enzymes involved in the beta-oxidation of unsaturated (ECI1/2) and long-chain (or very long-chain) fatty acids (HADHA/B and ACADVL) showed differential transcript and protein abundances (Fig. 3c) suggesting plausible buffering of protein levels beyond transcriptional regulation with IF.

With several of the IF-responsive proteins being enzymes (Supplementary Fig. 3), we next assessed if the protein abundance changes were transcriptionally driven by comparing their corresponding transcript abundances. We found a correlated increase in abundances of protein as well as mRNA of hydroxymethylglutaryl-CoA synthase (HMGCS2), the enzyme catalyzing the first step of ketogenesis, and pyruvate dehydrogenase isozyme (PDK4) that plays key regulatory roles in glucose and fatty acid metabolism, with the EOD regimen in comparison to the AL group (Fig. 3d, Tables S2 and S4). Phospholipid phosphatase 1 (PLPP1) that is involved in synthesis of glycerolipids and lipid uptake, however, showed a dramatic decrease in protein abundance particularly in the EOD group despite a modest increase in the corresponding transcript abundance. A group of enzymes modulating gene expression including $\mathrm{N}$-acetyltransferase NAT10 involved in histone acetylation and chromatin organization, ribonuclease RNASE4 participating in mRNA cleavage, and spliceosome-associated protein CWC27 involved in mRNA splicing showed exclusive increase only in the protein level with no changes in the mRNA level with IF. Changes in these proteins were more obvious in the EOD group and this supports extensive gene regulatory rewiring with extended fasting duration. Such discordant expression patterns at the mRNA and protein level could arise from post-transcriptional buffering that could potentially impact enzymes and kinases abundance and functions via post-translational modifications affecting protein stability or allosteric regulations that can alter activity.

Since changes in metabolism are closely linked with and regulated by circadian clocks, we also assessed if time-restricted feeding regimens of IF12 and IF16 mice during the day (light 
phase) displayed any altered circadian patterns compared to AL or EOD mice. For this, we analyzed the expression changes of core clock components at both the transcript and the protein levels (Supplementary Fig. 6). This analysis revealed that most of the clock gene transcripts displayed differential expression profile in comparison with the AL control group irrespective of the fasting regimen pattern (Table S4). Notably, both the IF12 and IF16 groups were similar to the EOD fasting group despite the time-restricted feeding during daytime. Except for the genes CRY1 and NR1D1 that showed differential expression between IF12 and EOD groups, all other clock genes showed consistent patterns of expression across the three intermittent fasting groups. In the corresponding proteome data, we only reliably quantified two clock components, CLOCK and CRY1, neither which showed significant differential protein expression among the fasting regimens.

\section{IF-induced functional transition network of heart proteome}

The distinct and overlapping proteomic profiles for the three IF regimens suggested a progressive molecular and cellular remodeling of the heart in response to increasing fasting durations. To address this possibility, we constructed functional transition networks to specifically compare proteome transitions from AL to IF12 to IF16 to EOD fasting (Fig. 4). Compared to AL, IF12 enhanced the processes that maintain the structural integrity of the heart such as adhesion, extracellular structure and cytoskeletal organization (Fig. 4a). We also observed increased expression of proteins involved in the immune and stress response, and endocytosis and exocytosis events in IF12. Along with major changes to circulatory process which displayed increased protein abundances, protein networks associated with heart rate regulation were also altered by IF12. As expected, proteins involved in metabolic processes such as sterol metabolism and transport, glucose utilization and protein synthesis were downregulated (Fig. 4a).

In contrast to the comparison between IF12 and AL, the functional transition from IF12 to IF16 involved fewer functional networks being upregulated with protein trafficking primarily affected (Fig. 4b). Multiple immune functional networks (NF- $\kappa B$ and Wnt signaling, innate immune response and $\mathrm{T}$ cell receptor signaling) and amino acid biosynthetic processes were downregulated in the IF12 to IF16 transition. Prolonged activation of NF- $\kappa$ B in immune cells occurs in several major cardiovascular diseases including cardiac hypertrophy and heart failure, and aberrant Wnt signaling contributes to cardiac hypertrophy (Foulquier et al., 2018; Gordon et al., 2011). Transition from IF12 to IF16 was also accompanied by changes in ion 
homeostasis and cardiac morphogenesis networks indicative of enhanced cardiac remodeling with increased fasting duration.

Transition from IF16 to EOD introduced a wide spectrum of changes in the heart proteome. EOD leads to epigenetic and genetic changes in addition to metabolic changes similar to those occurring in hearts of IF12 and IF16 mice (Fig. 4c). In particular, proteins associated with chromatin remodeling and organization, and those involved in histone and other protein modifications were increased in abundance. In parallel, proteins that regulate transcription initiation and termination, transcript splicing, and nuclear import were also upregulated in the hearts of EOD mice. The precise nature of the latter proteomic responses to EOD fasting, and their impact on heart physiology and resiliency are not known. In addition, we observed an increase in the mitotic and nuclear division regulatory networks. This was in contrast to reductions in cell cycle regulating pathways in the hearts of mice in the IF12 and IF16 groups. DNA repair and organelle assembly protein networks were also upregulated by EOD implying extensive cardiac tissue remodeling with EOD fasting. In addition to suppressed immune response and ion and sterol transport, the hearts of mice on EOD exhibited reduced abundance of components of protein networks involved in mitochondrial electron transport chain (ETC), pyruvate metabolism and ATP generation in comparison to IF16 mice (Fig. 4c). An additional protein network downregulated by EOD fasting is one that regulates blood coagulation, which might be expected to reduce the risk of pathological clot formation.

\section{Time-resolved protein network remodeling during IF}

To further elucidate how functional protein networks are remodeled in response to IF, we curated protein-protein interactions (PPI) of all IF-responsive proteins and explored their functional crosstalk (Fig. 5, Supplementary Fig. 7a-c and Table S6). Since many of the altered proteins were enzymes, in addition to physical interactions, we also considered functional interactions such as those that regulate gene expression, mediate enzymatic cascades or regulate protein phosphorylation. To this end, we used the Pathway Commons database, a compendium of pathway-centric biologically relevant interactions (Cerami et al., 2011). The interaction network included $\sim 81 \%$ (426) of the IF-responsive proteins with 2094 interactions connecting them. In order to decipher fasting time-dependent network remodeling, we mapped proteins that were differentially modulated across each fasting regimen in comparison to the AL group onto the network (Fig. 5a). We specifically marked those proteins that were altered with IF12 and those that only appeared with IF16 or EOD 
fasting. From the assembled networks, it was evident that network alterations were amplified in the IF16 and EOD groups compared to the IF12 group. Of all the changes that appeared with IF12 regimen, abundance changes in 78 proteins were conserved among all three fasting regimens (Table S6). Changes in the abundances of an additional 58 proteins appeared exclusively in the IF16 group, and abundance changes of another 141 proteins were evident in hearts of mice in both the IF16 and EOD IF groups. The EOD group exhibited the most extensive network remodeling with abundance changes of 142 proteins appearing exclusively with EOD (Table S6).

We next performed a cluster assessment using the ClusterONE graph-clustering algorithm (Nepusz et al., 2012) to identify densely connected network regions that may be functionally relevant. Our analyses highlighted several tightly connected clusters that included proteins responsive to different IF regimens and showed functional preference for specific metabolic processes (Fig. 5b and c, Supplementary Fig. 8, and Table S6). The cluster that constituted proteins associated with fatty acid beta-oxidation and BCAA catabolic processes was the most highly enriched cluster ( $\mathrm{p}$ value 5.98E-7) exemplifying the impact of IF on lipid oxidation. From the cluster, it was evident that many of the protein responses occurred with IF16 compared to IF12 and were maintained in EOD. Only four proteins (ACOT1, ACOT2, EHHADH and ALDOB) showed abundance changes exclusively with EOD in this cluster (Fig. 5b). Cardioprotective roles have been attributed to the fatty acyl-CoA thioesterase ACOT1 via its activation of PPAR $\alpha / \mathrm{PGC} 1 \alpha$ signaling (Yang et al., 2012), and our data pointed to increased expression of ACOT1 with extended fasting. Another lipid metabolismassociated cluster impacted by IF12, IF16, and more so by EOD, included proteins involved in cholesterol efflux, triglyceride homeostasis and chylomicron clearance ( $\mathrm{p}$ value $3.88 \mathrm{E}-5$ ) (Fig. 5c). The most prominent effects of IF on lipid metabolism were on the apolipoproteins APOA1/4, APOE and APOC1/2/3, all of which declined in the EOD group (Fig. 5c). There was also an increase in the level of NPC2, an intracellular transporter mediating export of cholesterol from lysosomes (Pfeffer, 2019), in the EOD group. On the other hand, protein level of PLTP, the increased activity of which is known to be associated with atherosclerosis, systolic dysfunction, obesity and diabetes (Chowaniec and Skoczyńska, 2018), was observed to be specifically suppressed in all three IF groups.

Apart from these lipid-linked clusters, we also observed other clusters differentially modulated in an IF group-dependent manner. For example, the RNA processing and splicing 
cluster ( $\mathrm{p}$ value 1.16E-5) included proteins that were modulated mostly with prolonged fasting (Supplementary Fig. 8 and Table S6). The cluster with proteins involved in ubiquitination and immune-related signaling exhibited changes in hearts from IF16 but not IF12 mice (p value 1.187E-5). Upregulated by IF16 within this cluster was LNPEP, a zincdependent aminopeptidase that shares interactions with various proteasomal components. This enzyme cleaves several peptide hormones and also catalyzes the conversion of angiotensinogen to cardioprotective angiotensin IV (AT4) (Stragier et al., 2008; Yang et al., 2011). From the network, we infer that other metabolic processes such as carbohydrate metabolism, glycolytic processes and TCA cycle show gradual modulations in protein abundance with increasing fasting duration (IF12 < IF16 < EOD) (Supplementary Fig. 8). We also identified PI3K signaling as one of the prominent clusters in the IF-responsive network (p value < 0.1) (Supplementary Fig. 8). Kinases including phosphatidylinositol kinase PIP4K2C, AKT1, PRKCD (PKC-delta) and EGFR were represented within this cluster. IF induced an increase in the abundance of AKT1, a kinase that is crucial for heart function and development in addition to insulin signaling (Hers et al., 2011).

Further interrogation of the protein interaction networks revealed IF-responsive hubs, which due to their centrality in the network may effectively relay signal during IF-induced network remodeling. In hearts from mice in the IF12 group, enzymes involved in glycogen catabolism (AGL, PGM1 and PYGM), glycolysis (HK1 and HK2), and alpha-linolenic metabolism (ACSL1, HSD17B4 and SCP2) were inferred as key proteins along with AKT1 and the receptor kinase EGFR. With the IF16 regimen, in addition to those involved in glycogen metabolism, proteins involved in heart contraction (CDC42 and DNM1L) were hub proteins. The GTPase CDC42, regarded as an antihypertrophic molecular switch that functions in protective signaling to reduce heart failure, was observed with enhanced expression. Hearts of mice in both IF16 and EOD groups exhibited enzymes involved in glucagon signaling, including LDHA, PYGM, PYGB, PKM and AKT1, as protein network hubs. In addition, the IF network for the EOD group highlighted paxillin as one of the hub proteins. Paxillin plays an essential role in regulating cardiac contractility and its redistribution has been reported in various cardiomyopathies (Hirth et al., 2016).

\section{Modified protein co-regulations in heart during IF}

Dynamic interactions among proteins coordinate cellular homeostasis and adaptive responses to environmental challenges. To decipher effects of IF on such protein associations, we 
investigated the protein co-regulation network of heart proteome for IF regimen-specific perturbations using the 'interactome mapping by high-throughput quantitative proteome analysis' (IMAHP) method (Lapek et al., 2017; Kosok et al., 2020) (Fig. 6). Accordingly, we first constructed a high-confidence correlation network by obtaining protein correlations between all pairs of quantified proteins and filtered for significant associations (adjusted $\mathrm{p}$ value $<0.01$ ) corresponding to a minimum Spearman's rank correlation coefficient of $\sim 0.82$ (Table S7a-b). The correlation network comprised a total of 44,966 associating protein pairs of which a majority (34,629 pairs) involved positive co-regulations between the associated proteins (Fig. 6a). Next, to identify protein associations perturbed in an IF group-dependent manner, we carried out outlier analysis to specifically derive associations that displayed significant deviation from the overall correlation across all co-regulated proteins. This analysis revealed higher deviations by $\sim 40 \%$ across all IF groups compared to the AL group, suggestive of dynamic modulation of protein associations with IF. Moreover, with median deviations of 584 and 241 for positive and negative co-regulating pairs, respectively, the EOD group exhibited the highest number of perturbed associations among all IF groups. Based on the perturbed associations for each IF group, we also identified consensus proteins that were consistently altered in protein associations across the different IF regimens using enrichment analysis (p value < 0.05) (Fig. 6a and Table S7c). Accordingly, we identified the greatest number of affected proteins in the IF16 and EOD groups, with the EOD group showing exclusive changes in 118 proteins and the IF16 group with 69 altered proteins. The IF12 group, on the other hand, displayed only a small number of perturbed associations similar to the AL group. Also, we noticed that many protein associations affected by IF16 were similarly affected by EOD fasting.

We next determined whether proteins affected by IF exhibit functional preferences (Fig. 6a). Proteins showing perturbations exclusively in the AL group compared to all of the IF group perturbation were associated with metabolic processes including carbohydrate and glycogen metabolism, oxidation-reduction and protein transport. These included phosphofructokinase (PFKL), lactate dehydrogenase A (LDHA), phosphoglycerate dehydrogenase (PHGDH), and kinases AKT2 and DTYMK. Unlike AKT1, which was found to be upregulated by IF, AKT2 did not vary in abundance with any IF regimen. A majority of the proteins displaying association perturbations in hearts of IF12 were localized to the mitochondria and are involved in glutathione metabolism and oxidation-reduction, suggesting that oxidative stress response mechanisms are impacted by IF12. Among these, we observed association 
modulation of glutathione S-transferase kappa 1 (GSTK1), which apart from regulating oxidative stress is also involved in multimerization of adiponectin, thus regulating its concentration and thereby indirectly modulating insulin sensitivity (Fig. 6a and Table S7c).

The IF16 regimen affected proteins in a wide variety of cellular processes including platelet activation, migration, translational control, fatty acid oxidation and Rap1 signaling (Fig. 6a and Table 7c). Several association modulations involved integrins, myosin and actin, suggestive of possible structural remodeling of the heart. Indeed, changes in some of these, including ITGB3 and ITGA2B have been linked to cardiac pathologies (Pillois and Nurden, 2016). Extensive modulation of heart protein association network was observed with EOD. While perturbations accompanying extended EOD fasting maintained some of the functional changes evident in IF16 hearts such as NADH oxidation, electron transport chain and protein translation, association alterations also occurred in processes involved in lipid and triglyceride metabolism. Importantly, we observed modulation of the PPAR signaling pathway by EOD fasting with key components PDPK1, APOA1, FABP3, PCK2 and SCP2 affected. Several of these proteins function as key regulators of glucose and lipid utilization, and play important physiological roles in the heart. This finding is interesting as PPAR modulators are currently being evaluated as therapeutic players for several cardiac diseases (Khuchua et al., 2018).

A consistent pattern across the inferred association perturbations was that most of these involved proteins in the metabolic pathways and were also localized to the mitochondria. To assess the functional implications of mitochondrial proteome responses to IF, we constructed a mitochondrial association network to identify the modulated associations that occurred in an IF regimen-specific manner. From the network it was evident that many of the perturbed proteins co-associate, particularly in the EOD group, and share associations relevant to an intricate orchestration of mitochondrial functional protein associations in response to IF (Fig. 6b and Table S7d). Proteins showing association perturbations with the mitochondrial proteins impacted by IF regulate BCAA catabolism, macroautophagy, oxidative phosphorylation, lipid oxidation, as well as AMPK and PPAR signaling. Strikingly, some of the proteins have also been implicated in cardiac muscle contraction and hypertrophic cardiomyopathy. For example, we found troponin T (TNNT2) constituting the troponin protein complex in the heart muscles that coordinates contraction to be modulated with prolonged EOD fasting. Two proteins of the sarcoglycan protein complex, SGCB and SGCD, which function to protect muscle fibers from injury, were also modulated. Among the 
mitochondrial proteins affected by IF, some including UQCRC1, METAP1D, SCP2, COA7, AFG3L2, FAM162A and COA4 were involved in associations spanning multiple functional processes suggesting coordinating rewiring of various pathways by mitochondrial functions. For instance, UQCRC1 shares IF responsivity with proteins involved in both fatty acid oxidation and carbohydrate metabolism. Overall, our data suggests the involvement of mitochondrial proteins in adaptive responses of the heart to IF.

\section{IF impacts phosphosignaling pathways in a cardioprotective manner}

To gain deeper insight into IF-induced signaling alterations in myocardial cells, we investigated changes in the phosphorylation landscape that accompanies IF (Figs. 7 and 8, Supplementary Fig. 9, and Table S8a). We enriched for phosphopeptides from heart tissue homogenates, and then profiled them using label-free phosphoproteome analysis. Among the different groups, we identified 2,931 proteins that were phosphorylated on 12,540 distinct sites. Of these, $\sim 80 \%$ were phosphorylated on serine, and the remaining $\sim 19 \%$ were phosphorylated on threonine, with only $1 \%$ being tyrosine phosphorylated. Peptides with single phosphorylation sites represented $81 \%$ of the phosphopeptides analyzed, and the remaining phosphopeptides displayed multiple phosphorylations. There was high reproducibility of phosphopeptide quantifications among the biological replicates with an average intensity correlation of 0.86 . Only $66 \%$ of the phosphosites corresponded with those curated in PhosphoSitePlus database (Hornbeck et al., 2012), indicating that our profiling revealed novel phosphorylation sites on many proteins some of which might possibly influence cardiac physiology.

To elucidate IF-induced changes in protein phosphorylation, we applied stringent filtering and focused only on 6,956 unique phosphorylation sites that were quantified in samples from at least two mice per diet group. Approximately $84 \%$ of these belonged to class I phosphorylation sites with a localization probability $\geq 0.75$, and overall displayed an average localization probability of 0.90 indicative of high phosphosite confidence. On assessing those sites that were significantly altered ( $\mathrm{p}$ value $<0.05)$ with $\mathrm{IF}$, we observed that phosphorylation changes varied with increasing duration of the fasting period with EOD exhibiting the greatest number of phosphosite abundance changes, similar to what was observed in the proteome (Fig. 7a and Table S8b). However, in contrast to IF-induced proteome remodeling wherein a majority of the regulated proteins displayed decreased abundance with IF, phosphoproteome profiling revealed increased phosphosite abundance on 
a majority of the differentially modulated phosphoproteins, and several of these were independent of proteome changes. This is intriguing as it points to differential exploitation of translational and post-translational mechanisms to remodel cardiac cells in response to IF. Site-specific phosphorylation changes with IF occurred on several classes of regulatory proteins including kinases, phosphatases and enzymes in metabolic pathways. For instance, we found phosphorylation changes on several MAPK associated kinases including MAPK1/3 and MAPK12, PKC family kinases including PKC-alpha, -beta and -theta, kinases that coordinate muscle contraction including MYLK, MYLK3 and PAK2, and sphingolipid signaling kinase SPHK2 (Table S8b). Importantly, enzymes involved in lipid metabolism such as ACOT1 and ACSL1, glycolytic enzyme triose phosphate isomerase (TPI1) and fumarate hydratase $(\mathrm{FH})$ in the TCA cycle, phosphorylase kinase (PHKB) involved in glycogen breakdown, all showed IF-responsive changes in phosphosite abundance emphasizing phosphorylation-dependent remodeling of energy homeostasis pathways with IF.

To dissect the functional implications of the modulated phosphoproteins in each IF regimen, we assessed ontology-based enrichment of processes and pathways (Fig. 7b and Supplementary Fig. 9a). Proteins associated with cardiac muscle contraction, actomyosin structure organization, myofibril assembly and heart developmental processes displayed altered phosphorylation, mostly showing increased phosphosite abundance with IF. In addition to structural remodeling, proteins regulating the force of muscle contraction and heart rate also displayed phosphorylation changes, emphasizing remodeling of signaling networks that underlie crucial heart functional adaptations. Phosphorylation of proteins that regulate cellular energy metabolism were affected by one or more IF regimens including proteins in the insulin signaling pathway and lipid metabolism. Compared to AL, IF16 induced the phosphorylation of proteins associated with AMPK signaling, a pathway that mediates adaptive responses of the heart to endurance training (Yavari et al., 2017) (Fig. 7b). Changes in the phosphorylation of proteins involved in FoxO signaling, which play regulatory roles in gluconeogenesis and insulin signaling, were evident only in the EOD group. Among the phosphosites most prominently increased in response to all three IF regimens were those in substrates for cyclic GMP-dependent protein kinase (PKG) (Fig. 7b). This is an interesting observation as cGMP-PKG signaling is known to mediate vasorelaxation and activation of this pathway is regarded as cardioprotective (Kukreja et al., 2012). Therefore, we further verified our proteomics observations by performing immunoblot analyses for key proteins involved in the cGMP-PKG pathway. We observed that the PKG protein level was increased 
along with a concomitant decrease in PDE5A protein level confirming the activation of cGMP-PKG pathway with IF (Fig. 7c).

While phosphorylation signatures are reflective of kinase and phosphatase activities, we noticed that only $2.8 \%$ of the phosphosites we quantified had experimentally validated kinases. To better understand the impact of IF on myocardial cell protein phosphorylation, we defined kinase-substrate relationships through motif mapping for each IF regimen. Accordingly, we focused on all the modulated phosphosites (ANOVA p value < 0.05), performed hierarchical clustering and identified motifs specifically enriched ( $\mathrm{p}$ value $<0.05$ ) within distinct phosphosite expression clusters. The clustering revealed discrete patterns of phosphorylation changes that occur in response to each IF regimen (Fig. 8a). The results suggest that each IF regimen impacts multiple kinases, including AMPK, AKT, PKC, PKA, and $\beta$-adrenergic receptor kinase. AMPK, a master regulator of cell metabolism that serves as an energy switch responsive to ATP depletion, emerged as one of the significantly enriched kinases in both the IF16 and EOD mouse hearts. In addition to AMPK, our phosphoproteome analyses identified activation of CAMK1/4 and CSNK1 kinases in response to IF16. In contrast, IF12 hearts only showed selective enrichment of the PLK1 PBD binding substrate motif. This is in agreement with our proteome data, which revealed more pronounced modulation of cellular signaling with IF16 and EOD compared to IF12.

Further analyses of the site-specific changes in protein phosphorylation that occurred in response to IF revealed several signaling cascades and metabolic processes. Our data revealed changes in phosphorylation of proteins in the insulin-signaling pathway including glucose transporter GLUT4 (encoded by SLC2A4) and adapter proteins insulin receptor substrates IRS1 and IRS2 that suggest increased insulin sensitivity in IF hearts (Fig. 8b). Notably IF resulted in reduced phosphorylation at serine 138 of IRS1, which would be expected to enhance insulin signaling. We also observed elevated phosphorylation changes at PGC1 $\alpha$ (encoded by PPARGC1A) at serine 615 and also predicted activation of AMPK with IF. Other IF-responsive protein phosphorylation sites included those on PRKAG2 (protein kinase AMP-activated non-catalytic subunit gamma 2), MAPK1 and MAPK3, SLC2A4 (GLUT4) and SORBS1, all of which are involved in the regulation of cellular energy metabolism. While proteome changes for PPAR signaling were greatest in hearts of EOD mice, alterations in phosphosite modulation were already prominent in IF16 hearts, suggesting that global changes in second messenger-mediated signaling pathways precede 
transcriptional adaptions to IF (Fig. 8c). In concordance with alterations in protein abundance, proteins involved in cholesterol metabolism and lipid transport exhibited changes in phosphorylation in response to one or more IF regimens (Fig. 8d and Supplementary Fig. 9b). Multiple sites on ACADL and ACADVL encoding long-chain and very long-chain specific acyl-CoA dehydrogenase were found to be modulated by IF. In addition to phosphorylation changes on APOE, that displayed decreased protein abundance, we observed changes on FABP3 that peaked in IF12 and IF16. Multiple phosphorylation alterations were also observed on oxysterol-binding protein lipid transporters (OSBP, OSBPL11 and OSBPL8) that bind cholesterol and regulate its efflux (Supplementary Fig. 9b). In addition, there was a striking increase in phosphorylation of epitopes on numerous proteins known to regulate heart development and cardiomyocyte contraction (Fig. 8e). These proteins included the cell adhesion molecules NCAM1 and plakophilin 4, the extracellular matrix molecule fibulin-2, and the dystrophin-interacting protein $\beta 1$-syntrophin (Ackermann et al., 2017; Grossmann et al., 2004; Kim et al., 2019; Zhang et al., 1995). Another phosphoprotein in this category is titin, a protein that is a crucial determinant of myocardial passive stiffness and whose malfunction is associated with various myopathies (Fig. 8e) (LeWinter and Granzier, 2014; Makarenko et al., 2004). In summary, our phosphoproteome analyses revealed that IF impacts many different signaling pathways known to influence the structural and functional plasticity of the heart.

\section{IF improves cardiac performance after stress}

With the enhanced cardiac resilience inferred from the omics data, we next investigated the effects of IF on heart function and dynamics in the IF mice compared to the AL mice. For this, we performed echocardiographic measurements in mice that had been maintained on $\mathrm{AL}$ or IF16 diets for 6 months. While no differences in heart function were observed between IF and AL groups, AL mice start to show signs of age deterioration, such as prolonged $\mathrm{E} / \mathrm{A}$ and shorten deceleration time (de Lucia et al, 2019) (Supplementary Fig. 10, Supplementary videos 1-4). To evaluate cardiac functional reserve, dobutamine stress tests were performed after basal parameter acquisition. As expected, administration of dobutamine resulted in significant inotropic, lusitropic, and chronotropic cardiac responses (Tyrankiewicz et al., 2013) in both groups. However, the increase in heart rate, as well as ejection fraction, fractional shortening and left ventricular dimensions after stress were significantly higher in IF16 mice compared with AL control mice (Table S9, Fig. 9a, Supplementary videos 1-4), suggesting that IF preserves myocardial functional reserve. To confirm these findings, strain 
analyses were performed. Longitudinal and radial strain significantly improved after dobutamine administration in IF16 mice but not in AL mice, suggesting that IF preserved systolic contractile reserve, which deteriorated with age in the AL mice (Table S9, Figure 9b).

\section{DISCUSSION}

Increasing evidence from animal and human studies suggests that IF reduces the risk for cardiovascular disease and bolsters myocardial function and resilience (Mattson and Wan, 2005; Mattson et al., 2017; Sutton et al., 2018). However, except for enhancement of parasympathetic tone (Griffioen et al., 2013; Mager et al., 2006) and upregulation of protein chaperones (Ma et al., 2019), the underlying mechanisms are unknown. Collectively, our multi-omics datasets provide a window into the molecular, cellular, structural and functional remodeling of the heart in mice adapted to IF eating patterns. To address the question of how the duration of the fasting period in specific IF regimens affects the molecular remodeling of the heart we compared molecular effects IF12, IF16 and EOD fasting. All three IF regimens affected pathways that regulate cellular carbohydrate, lipid and protein metabolism, cell-cell interactions and regulation of myocardial cell contractility. We generated a quantitative map of protein and phosphorylation changes that occur during different IF regimens by comprehensive curation of metabolic and pathway information to unveil time-resolved IFresponsive signaling topology (Fig. 10). A prominent cardioprotective pathway that was positively impacted by all three IF regimens was the cGMP-PKG pathway. This is a novel finding that has important implications for the application of IF regimens to patients with or at risk for cardiovascular disease. Our data also revealed differences amongst the IF regimens in other signaling pathways such as ERK, cAMP-mediated and PPAR, and it will be of interest to determine both the causes and consequences of these differences. Our study did not address the question of the time course of molecular remodeling of the heart after the onset of IF. Previous findings suggested that beneficial effects of IF on organ function and stress resistance require 2-4 weeks to be fully engaged (Bruce-Keller et al., 1999; Johnson et al., 2007; Liu et al., 2019; Mager et al., 2006). If and to what extent the molecular remodeling of the heart in response to IF reverts to the pre-IF state upon return to ad libitum feeding remains to be determined. 
There are many similarities in the effects of aerobic exercise and IF on the cardiovascular system that beg the question of the extent of overlap in the underlying molecular mechanisms. For example, both IF and regular exercise training increase parasympathetic tone and heart rate variability, and both can protect the myocardium against ischemic injury (Rengo et al., 2013; Voulgari et al., 2013). Moreover, both exercise and IF stimulate mitochondrial biogenesis in skeletal muscle (Drake et al., 2016; Marosi et al., 2018). Exercise training also stimulates mitochondrial biogenesis in myocardial cells (Bernardo et al., 2018). Studies of the effects of IF on the brain and skeletal muscle suggest that IF activates several major pathways that are also engaged by ischemic and metabolic preconditioning including upregulation of protein chaperones, anti-apoptotic proteins and autophagy, and suppression of the mTOR pathway and protein synthesis (Marosi et al., 2018; Mattson and Arumugam, 2018). Previous research has led to the development of several treatments that mimic one or more effects of exercise, caloric restriction and IF. Examples include agents that induce mild transient metabolic stress such as 2-deoxyglucose and mitochondrial uncoupling agents (Liu et al., 2015; Liu et al., 2019; Wan et al., 2003; Wan et al., 2004; Yu and Mattson, 1999), ketone esters (Cox et al., 2016; Kashiwaya et al., 2013) and mTOR inhibitors such as rapamycin (Sciarretta et al., 2014).

Our analysis of functional interactions and IF-induced alterations provides a global view of molecular and metabolic rewiring influencing various aspects of cardiac performance and bioenergetics. We observed IF-induced changes in expression of several enzymes functioning in key energy pathways, as well as kinases involved in cellular signaling. While IF triggered downregulation of several lipid metabolism enzymes, thioesterases that regulate fatty acid flux such as ACOT1, ACOT2, and ACOT7 were upregulated, especially in the EOD group. ACOT1, for instance, functions as a molecular rheostat to impede excessive fatty acid oxidation during fasting and dampens associated inflammation and oxidative stress by inducing protective PPAR $\alpha$ signaling (Franklin et al., 2017). Such an induction of PPAR $\alpha$ activity with IF is indeed supported by increased protein abundance of several of its transcriptional targets including HMGCS2, PDK4, ACOT2, EHHADH, and ALDH9A1. It is noteworthy that signaling alterations driving such PPAR-associated modulations were also evident from IF-induced phosphorylation changes including activation of PKA, one of the key modulators of PPAR transcriptional activity especially in the context of lipid oxidation during fasting and exercise (Lazennec et al., 2000). In addition to modulating fatty acid flux, PKA also triggers glycogen breakdown by activating phosphorylase kinase PHKB which in turn 
triggers glycogen phosphorylase to release glucose 1 phosphate from glycogen (Brushia and Walsh, 1999). Our data point to an increase in phosphorylation site abundance on PHKB with IF suggesting increased turnover of glycogen with IF. The PKA effect of IF was accompanied by changes in associated cAMP signaling which also showed enhanced activation with prolonged durations of fasting and a consistent increase in phosphorylation of adenylyl cyclase enzyme ADCY9 catalyzing the formation of cAMP across the IF regimens. The cAMP/PKA axis plays crucial modulatory roles in various aspects of cardiac physiology including cardiomyocyte contractility via excitation-contraction coupling (ECC), control of heart rate and downstream relay of $\beta$-adrenergic receptor signaling. The cAMP/PKA pathway is also known to be perturbed in instances of heart failure (Bockus and Humphries, 2015; Callaghan, 2016; Lissandron and Zaccolo, 2006). Taken together, the concerted protein changes in abundance and phosphorylation are all indicative of IF-induced rewiring of the PKA signaling axis.

AMPK emerged as another prominent signaling cascade that was activated with longer hours of fasting (IF16 and beyond). We observed that the substrates of AMPK were associated with multiple aspects of energy usage that are known to enhance the metabolic flexibility of the heart. Our phosphoproteome data revealed an AMPK-induced increase in phosphorylation of desnutrin/ATGL (encoded by PNPLA2) with prolonged fasting (Ahmadian et al., 2011). Phosphorylation at the specific site is associated with elevated triacylglycerol hydrolase activity resulting in enhanced lipolysis that can mobilize free fatty acids for increased energy demands during fasting (Ahmadian et al., 2011). TBC1D1, another substrate that is important in AMPK-induced GLUT4 translocation, also showed a consistent increase in functional phosphorylation with increasing fasting duration (Jessen et al., 2011). Disruption of GLUT4 trafficking is associated with impaired glucose uptake and ensuing insulin resistance (Mueckler, 2001), and the phosphorylation change on TBC1D1 and GLUT4 along with the predicted increase in AKT activity suggest that IF improves insulin sensitivity in the heart by modulating glucose uptake and resetting aberrations in insulin signaling via increasing phosphorylation of such proteins. The effects of insulin signaling are primarily mediated by AKT. We identified AKT1 to be differentially expressed based on protein abundance, AKT2 to have specific alterations in IF triggered association modulations, and also predicted AKT to be induced based on substrate signatures from our phosphoproteome data. Collectively, our data suggest that AKT may be an important mediator of heart cell responses to IF. In addition to its overall metabolic effect, AMPK was also inferred to activate other kinases such as 
casein kinase epsilon (encoded by CSNK1E) that constitutes a major component of mammalian oscillators regulating circadian rhythms (Lee and Kim, 2013).

Beyond effects on pathways involved in energy metabolism, our proteome data also suggested suppression of several pathways implicated in cardiac hypertrophy and dysfunction. For example, the Wnt signaling cascade was downregulated with increased fasting duration (Bastakoty et al., 2016; Zhao et al., 2018). In addition to protein abundance changes, our phosphoproteomic data identified targets of TNIK, an activator of Wnt signaling with altered sites with different fasting regimens. One of these sites, serine 707, which responds to activation of the angiotensin receptor, a key component of the renin-angiotensin system, was indeed downregulated with the IF16 regimen (Xiao et al., 2010). The downstream effects of angiotensin receptor signaling impact cardiovascular processes and are implicated in cardiac remodeling and dysfunction partially mediated by its induction of reactive oxygen species and oxidative stress (Ferrario, 2016; Zablocki and Sadoshima, 2013). Thus, a site-specific reduction in TNIK with IF is reflective of reduced angiotensin receptormediated signaling which is likely to be beneficial for cardiac output and function. Calineurin-NFAT is another signaling cascade that orchestrates hypertrophic and other pathological responses in the heart and we noted specific activation of its negative regulator DYRK1A with IF (Kuhn et al., 2009; Molkentin, 2004). DYRK1A is reported to attenuate pro-hypertrophic transcriptional programming favored by the NFAT family of transcription factors, thus contributing to cardioprotective mechanisms. With such modulations, we posit that IF activates various protective pathways by fine-tuning protein abundance and phosphorylation levels to alleviate processes that negatively impact cardiac physiology. In all, these highlight varying degrees of kinase and metabolic rewiring in the heart induced by different durations of IF with most protective effects accompanying prolonged fasting. Such protein modulations will likely improve metabolic flexibility of the heart to allow it to shift between energy sources to meet its energy demand and maintain resilience.

Although our data provide a comprehensive molecular snapshot of the changes that accompany various courses of IF, a limitation of our study is that our landscape is reflective of net changes occurring across the entire heart. Thus, we can draw no conclusions about specific changes within the different cell types that comprise the heart. Such a limitation is exaggerated particularly for the transcriptome analysis as cardiomyocytes account for only $30 \%$ of cells in the mammalian heart and yet account for the majority of the cardiac mass 
(Zhou and $\mathrm{Pu}, 2016$ ). Knowledge of cell type-specific responses to IF will therefore be important to delineate distinct responses of heart cells to various IF regimens, and future studies exploring single-cell sequencing will add new dimensions to our understanding of cardiac dynamics with IF.

Several aspects of our study will require further experimentation for clarification. The timing of fasting cycles in this study may not promote synchronization of the natural circadian rhythm of nocturnal rodents, which may impact the potential for translating these to other model systems and understanding human patho-physiology. It has been established that timerestricted feeding/intermittent fasting cycles impact and can reset secondary tissue-specific peripheral clocks independent of the master circadian clock that is generally phase-locked to light-dark cycles (Damiola et al., 2000; Hara et al., 2001; Stokkan et al., 2001). While such mechanisms have been well documented in the context of liver and hepatic clocks, the impact of feeding on cardiac clock rhythmicity, however, remains to be deciphered. Evidences from our data suggest that fasting regimens affect circadian oscillations in heart cells, at least in part, independently of the central light-dark cycle-responsive clock. In addition, our analysis of cardiac function only included the AL and IF16 groups. Echocardiographic analysis of the EOD group may provide significant information on the effect of IF as the EOD group had the largest effect sizes from multi-omics analyses. It is noteworthy that previous studies established that the EOD regimen leads to hearts with thinner walls and a lower left ventricular end-diastolic volume (LVEDV) (Ahmet et al., 2005) and lower blood pressure and heart rate (Wan et al., 2003a) than those of control animals. The latter effects of EOD were associated with cardioprotection in a rat model of myocardial infarction (Ahmet et al., 2005). Furthermore, our multi-omics study was limited to mice that were 6 to 9 months old, and it is therefore difficult to draw conclusions as to the effects IF in ageing heart functions.

All three IF groups maintained similar average body weights throughout the study period and were significantly lower than the AL group. Therefore, the different transcriptomes, proteomes and phosphoproteomes observed among the three IF groups were independent of body weight. Our data suggest that overlapping but distinct molecular adaptations of the heart to different intermittent fasting regimens. Altogether, our multi-omics study elucidates molecular mechanisms by which IF improves cardiac health and counteracts disease processes. This information may lead to novel pharmacological approaches to prevent and treat cardiovascular diseases. 


\section{EXPERIMENTAL PROCEDURES}

\section{Animals and IF procedures}

C57BL/6NTac male mice were purchased at 2 months of age (InVivos, Singapore) and housed in the animal facility at the National University of Singapore. The animals were exposed to light from 07:00-19:00 (12 h light: $12 \mathrm{~h}$ dark cycle) and were fed with pellets for rodents which consisted of $58 \%, 24 \%$, and $18 \%$ of calories originating from carbohydrate, protein, and fat, respectively (Teklad Global 18\% Protein rodent diet \#2918; Envigo, Madison, WI, USA). Water was provided ad libitum to all dietary groups. The National University of Singapore Animal Care and Use Committee approved all experimental procedures performed in vivo. At the age of 3 months, the mice were randomly assigned to the four different dietary intervention groups: IF12, IF16, EOD (Every Other Day fasting), and AL group as a control ( $\mathrm{N}=20$ /group). The mice in the IF12, IF16, and EOD groups were fasted for 6 months daily 12 h (19:00-07:00), 16 h (15:00-07:00), or 24 h (a whole day fasting followed by a whole day feeding). The AL group was supplied with food pellets ad libitum. The body weight was regularly measured as well as the blood glucose and ketone levels using FreeStyle Optium Neo system with FreeStyle Optium blood glucose and ketone test strips (Abbott Laboratories, Berkshire, UK). After 6 months, animals were anesthetized and euthanized. EOD mice were euthanized on a food-deprivation day. All mice were euthanized under isoflurane anesthesia between 7 a.m. and noon. All mice were perfused with cold PBS. The heart samples were collected and kept at $-80^{\circ} \mathrm{C}$ until further use.

\section{Sample preparation for proteome analyses}

Frozen tissue sample was crushed in liquid nitrogen prior to lysis with 6M urea (SigmaAldrich), 2M thiourea (Invitrogen) and 20mM Hepes. Lysate was collected after centrifugation at $20,000 \mathrm{~g}$ for $20 \mathrm{~min}$ at $15^{\circ} \mathrm{C}$ and protein measurement was made using Pierce $^{\mathrm{TM}}$ 660nm Protein Assay Reagent (Thermo Scientific ${ }^{\mathrm{TM}}$ ) according to the manufacturer's protocol. $50 \mu \mathrm{g}$ of protein was used to prepare for each TMT label. For reference channel of 126-TMT label, a total of $50 \mu \mathrm{g}$ protein pooled from the 4 ALs was used. Reduction was performed with final concentration $5 \mathrm{mM}$ dithiothreitol (Sigma-Aldrich) incubation for 30min. This was followed by alkylation with final concentration $10 \mathrm{mM}$ iodoacetamide (Sigma-Aldrich) for 30min incubation in dark. Digestion was carried out using lysyl endopeptidase digestion (Wako Chemicals $\mathrm{GmbH}$ ) with enzyme: protein = 1:100 at $37^{\circ} \mathrm{C}$ overnight. The urea in the lysis buffer was then diluted to final concentration of $1 \mathrm{M}$ with $50 \mathrm{mM}$ ammonium bicarbonate followed by trypsin digestion (Promega) with enzyme: 
protein $=1: 50$ at $37^{\circ} \mathrm{C}$ for 8 hours. Digested peptides were desalted using $1 \mathrm{ml}$ Empore $\mathrm{C} 18$ cartridges $(3 \mathrm{M})$ before isobaric labeling.

\section{Sample preparation for phosphoproteome analyses}

IMAC was employed for selective enrichment of phosphorylated peptides. IMAC beads were prepared as described (Anal Chem 81, 4566-4575 (2009)) with minor modifications. Briefly, Ni-NTA agarose conjugates (Qiagen) was rinsed thrice with MilliQ water and incubated with 100mM EDTA pH 8.0 for 30minutes at RT to strip the nickel. Residual nickel was rinsed thrice with MilliQ water, followed by incubation with $100 \mathrm{mM}$ iron chloride solution for 3045 minutes. The resin was then washed thrice with MilliQ water followed by $80 \%$ acetonitrile / $0.1 \%$ trifluoroacetic acid. The tryptic peptides were then enriched using the iron chloride treated IMAC beads. Tryptic peptides were reconstituted in $50 \%$ acetonitrile / $0.1 \%$ trifluoroacetic acid, followed by 1:1 dilution with $99.9 \%$ acetonitrile / $0.1 \%$ trifluoroacetic acid. The peptides were incubated with $10 \mu 1$ of IMAC beads for 30 minutes with end-overend rotation and the beads were subsequently loaded onto self-packed C18 stage tips (Rappsilber et al., 2007) pretreated with $200 \mu 1$ methanol, washed with $50 \%$ acetonitrile / $0.1 \%$ formic acid and equilibrated with $1 \%$ formic acid. Following loading onto the stage tip, the IMAC beads were washed with $80 \%$ acetonitrile / $0.1 \%$ trifluoroacetic acid and $1 \%$ formic acid. The phosphopeptides were eluted from the IMAC beads onto C18 membranes

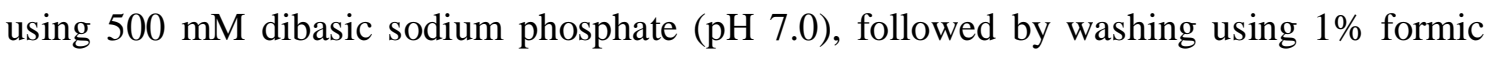
acid. The phosphopeptides were stored on the stage tip until they were ready to be analysed by LC-MS. The phosphopeptides were eluted from the C18 membranes with 50\% acetonitrile / $0.1 \%$ formic acid, dried using a speedvac and reconstituted in $0.1 \%$ formic acid for LC-MS analysis.

\section{Isobaric labeling}

All samples were ran in a total of two sets of TMT10plex ${ }^{\mathrm{TM}}$ Isobaric Label Reagent Set (Thermo Scientific ${ }^{\mathrm{TM}}$ ) and samples were assigned to each TMT set (9 samples to 9plexes and an internal reference sample made up of a mixture of the ALs) by randomization. Isobaric labelling was performed according to the manufacturer's protocol. Briefly, TMT label was reconstituted in anhydrous acetonitrile (Sigma-Aldrich) before mixing with respective peptides and incubated for 1hour. Reaction was quenched using final 5\% hydroxylamine (Merck) in triethylammonium hydrogen carbonate buffer (Fluka) for 15min. Labeled peptides 
were mixed before vacuum-dried and fractionated using basic reverse phase chromatography (Shimadzu NexeraXR high performance liquid chromatography). Fractions were combined orthogonally into 15 fractions and dried using a vaccum centrifuge.

\section{Liquid chromatography and mass spectrometry}

For proteome analysis, peptides were reconstituted in $0.1 \%$ formic acid for analysis on Thermo Easy nLC 1000 that was connected to Q Exactive ${ }^{\mathrm{TM}}$ Plus Hybrid QuadrupoleOrbitrap $^{\mathrm{TM}}$ Mass Spectrometer (Thermo Scientific ${ }^{\mathrm{TM}}$ ). The trap column used was C18 Acclaim PepMap ${ }^{\mathrm{TM}} 100$ of $5 \mu \mathrm{m}, 100 \mathrm{~A}, 100 \mu \mathrm{m}$ I.D. $\mathrm{x} 2 \mathrm{~cm}$ and the analytical column was PepMap $^{\text {TM }}$ RSLC C18, $2 \mu \mathrm{m}, 100$ A, $75 \mu$ m I.D. x $50 \mathrm{~cm}$. The LC solvent A comprised of 0.1 $\mathrm{M}$ formic acid in 2\% acetonitrile and LC solvent B comprised of $0.1 \mathrm{M}$ formic acid in $95 \%$ acetonitrile. The gradient was as follows: $5-50 \%$ solvent B in $180 \mathrm{~min}$; 50-100\% solvent B in $10 \mathrm{~min} ; 100 \%$ solvent $\mathrm{B}$ for $10 \mathrm{~min}$ at the flow rate of $200 \mathrm{~nL} / \mathrm{min}$. The mass spectrometer was set in the data dependent acquisition mode. Full scan MS spectra (m/z 380 - 1600) were acquired with a resolution of $\mathrm{R}=140,000$ at an AGC target of $3 \mathrm{e} 6$, and a maximum injection time of $60 \mathrm{~ms}$. Top 20 most intense peptide ions in each MS scan were sequentially isolated to an ACG target value of 1e5 with resolution of 35,000 and fragmented using a normalized collision energy of 30 at MS2 level with fixed first mass at 110 and isolation window of 1.2 $\mathrm{m} / \mathrm{z}$.

For phosphoproteome, reconstituted peptides were analysed using an EASY-nLC 1000 (Proxeon, Fisher Scientific) attached to a Q-Exactive (Thermo Fisher Scientific). Peptides were enriched using a C18 precolumn and separated on a $50 \mathrm{~cm}$ analytical column (EASYSpray Columns, Thermo Fisher Scientific) at $50{ }^{\circ} \mathrm{C}$ using a 265 min gradient ranging from 0 to $40 \%$ acetonitrile $/ 0.1 \%$ formic acid, followed by a 10 min gradient ranging from 40 to 80 $\%$ acetonitrile / $0.1 \%$ formic acid and maintained for $10 \mathrm{~min}$ at $80 \%$ acetonitrile $/ 0.1 \%$ formic acid. Survey full scan MS spectra (m/z 310-2000) were collected with a resolution of $r$ $=70,000$, an AGC target of $3 \mathrm{e} 6$ and a maximum injection time of $10 \mathrm{~ms}$. Twenty of the most intense peptide ions in each survey scan with an intensity threshold of 10,000, underfill ratio of $1 \%$ and a charge state $\geq 2$ were sequentially isolated with a window of 2 Th to a target value of 50,000 with a maximum injection time of $50 \mathrm{~ms}$. These were fragmented in the high energy collision cell by dissociation, using a normalized collision energy of $25 \%$. The 
MS/MS was acquired with a starting mass of m/z 100 and a resolution of 17,500 and dynamic exclusion of duration of $15 \mathrm{~s}$.

\section{Total RNA extraction and quality control for transcriptome analyses}

EZ-10 Total RNA Mini-Prep Isolation kit (Bio Basic, Canada) was used according to the kit's instructions to extract total RNA from the heart samples. To assess the quality of the extracted total RNA, agarose gel electrophoresis and Agilent Bioanalyzer 2100 system (Agilent, Santa Clara, CA, USA) were used. All RNA samples had high quality with integrity numbers $>6.9$ for total RNA.

\section{cDNA library preparation and RNA sequencing}

Poly-T oligo-attached magnetic beads were used to purify the mRNA from total RNA. Then, fragmentation buffer was added to the mRNA. First-strand cDNA was subsequently synthesized using random hexamer primer and M-MuLV reverse transcriptase (RNase $\mathrm{H}-$ ) (New England BioLabs, Ipswish, MA, USA). Next, second-strand cRNA synthesis was performed using DNA polymerase I and RNase H. AMPure XP beads (Beckman Courter Life Sciences, Indianapolis, IN, USA) was used to purify the double-stranded cDNA. By using exonuclease/polymerase activities, the remaining overhangs of the purified double-stranded cDNA were transformed into blunt ends. The 3' ends of the DNA fragments were adenylated followed by ligation of the NEBNext adaptor with hairpin loop structure to prepare for hybridization. The library fragments were purified with AMPure XP system to select cDNA with the base pair length of 150-200. Lastly, the library was amplified by polymerase chain reaction (PCR) and the PCR products purified using AMPure XP beads. The HiSeq ${ }^{\mathrm{TM}} 2500$ platform (Illumina, San Diego, CA, USA) was used to conduct the high-throughput sequencing.

\section{Transcriptome data mapping and differential expression analyses}

The RNA sequencing results from the HiSeq system were output as quality and color space fasta files. The files were mapped to the Ensembl-released mouse genome sequence and annotation (Mus musculus; GRCm38/mm10). Bowtie v.2.0.6 Indexes were used to build the reference genome and TopHat v.2.0.9 with mismatch parameter limited to 2 was used to align the paired-end clean reads to the reference genome. 48.5 million clean reads were generated per sample and 43.7 million reads ( $90.1 \%$ of total clean reads) per sample were mapped to the reference genome. HTSeq v.0.6.1 was used for the quantification of gene expression level to 
count the read numbers mapped of each gene. Then FPKMs of each gene was calculated based on the length of the gene and reads count mapped to the same gene. DESeq R Package v.1.10.1 was used to perform differential expression analysis. To control the false discovery rate, the p-values resulted from the analysis were adjusted to q-values using the Benjamini and Hochberg's approach. Genes with q-value $<0.05$ found by DESeq were assigned as differentially expressed.

\section{Heat map generation and functional enrichment analyses}

$\mathrm{R}$ and the $\mathrm{R}$ package heatmap3 were used along with the $\log 2$ fold change output from Edge R v.3.2.4 to create heatmaps of DEGs. To estimate the possible biologic functional changes derived from the gene expression changes, gene ontology (GO) and Kyoto Encyclopedia of Genes and Genomes (KEGG) pathway enrichment analyses were conducted. GO enrichment analysis was implemented by the GOseq R package in which gene length bias was corrected. For KEGG pathway enrichment analysis, we used KEGG Orthology Based Annotation System (KOBAS) software to test the statistical enrichment. GO terms and KEGG pathways with the q-value less than 0.05 were considered significantly enriched by DEGs.

\section{Proteome and phosphoproteome data processing}

For 10plex-TMT labeled proteome analysis, raw MS data was processed using Proteome Discoverer 2.2 (Thermo Scientific ${ }^{\mathrm{TM}}$ ). Database search was performed using the integrated Sequest HT search engine against the Uniprot mouse FASTA database (release January 2018) for tryptic peptides with maximum of two missed cleavage sites, MS and MS/MS mass tolerance of $10 \mathrm{ppm}$ and $0.02 \mathrm{Da}$, respectively. Searches included cysteine carbamidomethylation and TMT-modifications at peptide $\mathrm{N}$-termini and lysine residues as fixed modifications, and protein N-terminal acetylation and methionine oxidation as dynamic modifications. Peptide and protein identifications were performed at false discovery rate $(\mathrm{FDR})<0.01$. For phosphoproteome analysis, raw MS data was processed using MaxQuant version 1.6.0.1 (Tyanova et al., 2016a). The Andromeda search engine was employed to search against the UniProt mouse FASTA database (release January 2018). For identification of proteins, peptides and modifications, the FDR was set at 0.01 allowing a maximum of two missed cleavages as well as an initial mass tolerance of $4.5 \mathrm{ppm}$ for precursor ions and 20 ppm for fragment ions. The search was performed with phosphorylation of sites serine, threonine and tyrosine (STY), oxidation of methionine and acetylation of protein N-term as variable modification, cysteine carbamidomethylation as fixed modification and trypsin as 
cleaving enzyme. Label-free quantitation (LFQ) was performed using the MaxLFQ algorithm as implemented in MaxQuant with minimum ratio count set to 1 and match between run feature enabled to transfer peptide identifications across MS runs (match time window $=2$, alignment time window $=20)($ Cox et al., 2014). A minimum Andromeda score of 40 was used for phosphopeptide identification.

\section{Proteome and phosphoproteome data analyses}

For TMT-based proteome analysis, the common internal reference, pooled AL sample, at the 126 TMT-reporter channel of each TMT set was used for normalization across the two sets. Protein abundances across all the channels were first normalized for equal total protein intensities before obtaining $\log 2$ ratios of each protein against the normalized intensity of the respective reference channel. After combining the two TMT sets, only those proteins that were quantified in at least three biological replicates within each group were retained for subsequent analysis. For differential expression analysis of proteins regulated with IF, the replicates from the two TMT sets were grouped, and one-way analysis of variance (ANOVA) was performed with permutation-based FDR cutoff set at 0.1 , followed by post hoc tests to test for pairwise significance testing ( $\mathrm{p}$ value $\leq 0.05$ ). Protein annotations for gene ontology (GO) terms (biological process, cellular component and molecular function) and KEGG pathways were obtained and functional enrichment analysis was performed using Fisher exact test $($ FDR $\leq 0.05)$ for the modulated clusters. In addition, 1-D annotation enrichment analysis as implemented in Perseus was performed for testing preference of protein expression values to be higher or lower in comparison to the global distribution for specific functional groups (Tyanova et al., 2016b). The FDR was controlled at a threshold of 0.05 , and for each functional group a score was assigned, with scores those higher than zero and close to 1 confirming to positive enrichment, and those lesser than zero, and close to -1 , represent negative enrichment (Subramanian et al., 2005). To construct functional transition network over the different IF regimen, gene-set enrichment analysis (GSEA) was performed on the proteome data using all curated mouse GO biological processes and pathways. Pairwise comparisons of the different groups were performed and statistical significance of the enrichment score was assessed by permutation test. Based on similarity between enriched gene sets ( $\mathrm{p}$ value $<0.01$ ), EnrichmentMap plugin as implemented in Cytoscape was used to visualize all overlapping gene set clusters (Merico et al., 2010). All heat maps corresponding to functional categories were visualized using internal log-transformed reference normalized abundance value for each protein (Plubell et al., 2017). 
For phosphoproteome analysis, the label-free intensities of all identified phosphopeptides were log-transformed and only those phosphopeptides reliably quantified in at least two biological replicates of each group were retained for all subsequent analyses. Using the Perseus software environment, missing values were imputed to represent intensities of low abundant proteins based random numbers drawn from a normal distribution in each sample (Tyanova et al., 2016b). Phosphorylation sites differentially regulated between the groups were identified using one-way ANOVA ( $p$ value $\leq 0.05$ ). In addition, pairwise comparison using Student's t test was performed to assess differential regulation of phosphorylation sites in comparison to the AL group ( $\mathrm{p}$ value $\leq 0.05$ ). Biological processes and KEGG pathways for differentially altered phosphoproteins were derived from DAVID (Huang da et al., 2009). For upstream kinase enrichment analysis, kinase substrate motifs were extracted from human protein reference database (HPRD) and enrichment analysis was performed using Fisher exact test for altered phosphosite clusters across the groups.

\section{Comparison of proteome and transcriptome data}

For evaluation of proteome against the transcriptome, only those transcripts with corresponding protein quantification were used. Correlation analysis was performed using FPKM as a proxy for mRNA abundance and normalized protein abundance derived from TMT experiments for protein quantification. Data were log-transformed prior to visualization. For comparison of transcriptome and proteome changes across different IF groups, logtransformed fold changes relative to AL group were used as input to perform two dimensional (2-D) functional enrichment analyses as implemented in Perseus (Tyanova et al., 2016b). The respective annotation matrix of KEGG pathways and GO terms (biological process) of both the transcriptome and proteome were compared for significant bias to larger or smaller expression values in comparison to overall distribution. The FDR was controlled at a 0.05 threshold and position score was assigned to denote enrichment at higher $(0<$ score $<1)$ and lower $(-1<$ score < 0$)$ abundances. Scatter plot was constructed based on the positional scores and functional categories were highlighted.

\section{Protein-protein interaction network analyses}

Functional connectivity between significantly altered proteins were explored using proteinprotein interactions curated in the Pathway Commons database, which compiles pathway and interaction data from multiple resources (Cerami et al., 2011). In addition to direct physical 
interaction between proteins, associations that represent functional interactions such as those that regulate expression, control protein state change and catalysis, or mediate complex formation were also considered. The IF-altered functional network encompassed interactions relevant to all metabolic, regulatory as well as signaling pathways. Duplicate interactions were removed to remove redundancy, and the network was populated with information on the regimen-specificity of altered proteins to obtain time-resolved functional networks. Cluster assessment was performed using ClusterONE implemented in Cytoscape to identify densely connected regions within the network (p value < 0.1) (Nepusz et al., 2012; Shannon et al., 2003). The functional biases of the identified clusters for ontology and pathways were derived from DAVID (Huang da et al., 2009). Finally, topological parameters of the network were assessed for node centrality and degree distribution.

\section{Perturbation association network analyses}

A correlation matrix was obtained based on the quantitative proteome from all groups using Spearman's rank correlation. The correlation coefficients and $\mathrm{p}$ values were calculated for all pairs of proteins and multiple testing was controlled using Benjamini-Hochberg correction. Proteins pairs with adjusted $\mathrm{p}$ value $\leq 0.01$ were filtered to retain only those highly confident protein-protein associations (absolute correlation coefficient $\sim 0.82$ ) pertaining to both positive and negative correlations. Unweighted correlation networks were assembled using the high confident associations, and following the 'interactome mapping by high-throughput quantitative proteome analysis' (IMAHP) method (Lapek et al., 2017), specific deviations of each sample from the correlations were evaluated using Mahalanobis distance followed by outlier analysis using Grubbs's test ( $p$ value $\leq 0.1$ ) to identify perturbed associations in each samples. To identify consensus-perturbed proteins among different dietary groups, hypergeometric test was performed against the background of all perturbations ( $p$ value $\leq$ 0.05). Functional assessment of perturbed proteins in each group for processes and pathways were derived from DAVID (Huang da et al., 2009). For construction of mitochondrial perturbation network, we extracted those perturbed proteins annotated "mitochondria" across all groups, along with their regimen-specific co-regulated associations to be visualized as a network with functional annotations.

\section{Immunoblot analysis}

Frozen heart tissue samples ( $\mathrm{n}=7$ in each experimental group) were homogenized using a tissue tearor (BioSpec, Model: 985370) with protein and phosphatase inhibitors and Tissue 
Protein Extraction Reagent (Thermo Scientific, 78510) as the lysis buffer. The heart tissue samples were then combined with 2 x Laemelli buffer (Bio-Rad Laboratories, Inc., Hercules, CA, USA). Protein samples were then separated on 7.5 to $20 \% \mathrm{v} / \mathrm{v}$ sodium dodecyl sulfate (SDS) gels. The SDS-PAGE gels were transferred onto nitrocellulose membranes to probe for proteins. Next, the nitrocellulose membranes were incubated with the following primary antibodies: VASP (Santa Cruz Biotechnology, A-11), PDE5A (Santa Cruz Biotechnology, D3), PKG1 (Cell Signalling Technology, C8A4), MLCK (Sigma-Aldrich, M7905), pan-NOS (Santa Cruz Biotechnology, NOS-3F7-B11 B5), MYPT1 (Santa Cruz Biotechnology, C-6), pMYPT1 (Santa Cruz Biotechnology, F-11), Vinculin (Cell Signalling Technology, 13901S) and $\beta$-actin (Sigma-Aldrich, A5441) overnight at $4{ }^{\circ} \mathrm{C}$ with agitation. After primary antibody incubation, membranes were washed three times with 1xTBST before incubating with horseradish peroxidase (HRP)-conjugated secondary antibodies (Goat Anti-Rabbit - Cell Signalling Technology, Danvers, MA, USA; Goat Anti-Mouse - Sigma-Aldrich, St. Louis, MO, USA) for $1 \mathrm{hr}$ at $24{ }^{\circ} \mathrm{C}$ with agitation. The membranes were then washed three times (10 min each) with 1xTBST. The substrate for HRP, enhanced chemiluminescence (ECL) (BioRad Laboratories, Inc., Hercules, CA, USA) was applied for $3 \mathrm{~min}$ before the membranes were imaged using the ChemiDocXRS+imaging system (Bio-Rad Laboratories, Inc., Hercules, CA, USA). Quantification of proteins was conducted using Image J software (Version 1.52a; National Institute of Health, Bethesda, MD, USA), where protein densitometry was expressed relative to the densitometry of the corresponding $\beta$-actin or Vinculin respectively.

\section{Echocardiographic assessment of cardiac function}

At end-point of the experiment, myocardial function and cardiac dynamics were investigated by ultra-high frequency ultrasound for small animals (Vevo 3100 Preclinical Imaging System, FujiFilm VisualSonics, Canada) using the MicroScan Transducer MX550D (32-55MHz, Centre Transmit $40 \mathrm{MHz}$, Axial Resolution $40 \mu \mathrm{m}$ ) as previously described (Liehn et al., 2013; Tyrankiewicz et al., 2013). The Biomedical Sciences Institute Singapore Institutional Animal Care Committee approved all experiments. Mice were anesthetized with $1.5 \%$ isoflurane via mask and placed in supine position on a warming pad with integrated electrodes for continuous ECG monitoring. Body temperature was monitored via a rectal temperature probe. After complete removal of the hairs (hair removal cream), warmed up ultrasound gel was spread on the chest to avoid the formation of air bubbles and to ensure optimal sound quality. If needed, isoflurane was adjusted during the acquisition to maintain a relatively constant heart rate over 300 beats per minutes (bmp). Two-dimensional and M- 
Mode were acquired in long and short axis using ECG-triggered image acquisition to assess ejection fraction, fractional shortening, cardiac output, stroke volume and ventricular dimensions. The average between the values obtained from the long and short axis acquisitions was considered for all parameters. Strain analysis was performed in long axis to measure the global longitudinal strain (GLS), strain rate and radial strain (Lindsey et al., 2018). Pulsed wave (PW) Doppler was used to measure the velocity of blood flow through the aortic, pulmonal, mitral and tricuspidal valves. Septal mitral annular velocity was measured using tissue doppler echocardiography. To uncover a potential cardiac functional reserve, a dobutamine-induced stress was performed after basal parameter acquisition. Dobutamine was administrated intraperitoneally, in a single injection at $20 \mathrm{mg} / \mathrm{kg}$ (Tyrankiewicz et al., 2013). After the maximum increase in heart rate (generally within 4-5 minutes), two-dimensional and M-Mode acquisitions in long and short axes were repeated to assess ejection fraction, fractional shortening, cardiac output, stroke volume, ventricular dimensions and strain in stress conditions. 


\section{AUTHOR CONTRIBUTIONS}

Study Conception \& Design: T.V.A., and J.G; Investigation-Performed Experiment or Data Collection: T.V.A., A.A.S., E.A.L., J.K., H.L.F.S., D.T.Z.H., C.R., D.Y.W.F., L.P., D.C.M., G.Y.Q.N., S.H.B., K.M., M.G., and R.R.S; Computational \& Statistical Analysis: T.V.A., A.A.S., J.G., and J.G; Data Interpretation \& Biological Analysis: T.V.A., A.A.S., E.A.L., J.G., C.G.S., D.G.J., G.D., M.P.M., and J.G; Writing-Manuscript Preparation: T.V.A., A.A.S., E.A.L., J.G., H.L.F.S., C.G.S., M.G., K.M., M.P.M., D.G.J., G.R.D., R.R.S. and B.K.K; Supervision \& Administration: T.V.A., and J.G.

\section{ACKNOWLEDGMENTS}

This work was supported by ODPRT, National University of Singapore, National Medical Research Council Research Grants (NMRC-CBRG-0102/2016 and NMRC/OFIRG/0036/2017), Singapore and Agency for Science, Technology and Research, Singapore and the start up fund to TVA from La Trobe University, Melbourne, Australia. Fig. $7 \mathrm{c}$ in this article was created using BioRender.

\section{DATA AVAILABILITY}

The mass spectrometry proteomics data have been deposited to the ProteomeXchange Consortium via the PRIDE partner repository with the dataset identifier PXD015452. The data can be accessed using Username: reviewer34563@ebi.ac.uk Password: Y3btQBCL. High-throughput RNA sequencing data from this manuscript have been submitted to the NCBI Sequence Read Archive (SRA) under accession number GSE133290. 


\section{REFERENCES}

Ackermann, M.A., Petrosino, J.M., Manring, H.R., Wright, P., Shettigar, V., Kilic, A., Janssen, P.M.L., Ziolo, M.T., and Accornero, F. (2017). TGF-betal affects cell-cell adhesion in the heart in an NCAM1-dependent mechanism. J Mol Cell Cardiol 112, 4957.

Ahmadian, M., Abbott, M.J., Tang, T., Hudak, C.S., Kim, Y., Bruss, M., Hellerstein, M.K., Lee, H.Y., Samuel, V.T., Shulman, G.I., et al. (2011). Desnutrin/ATGL is regulated by AMPK and is required for a brown adipose phenotype. Cell Metab 13, 739-748.

Ahmet, I., Tae, H.J., de Cabo, R., Lakatta, E.G., Talan, M.I. (2011). Effects of calorie restriction on cardioprotection and cardiovascular health. J. Mol. Cell. Cardiol. 51, 263271 .

Ahmet, I., Wan, R., Mattson, M.P., Lakatta, E.G., Talan, M. (2005). Cardioprotection by intermittent fasting in rats. Circulation 112, 3115-3121.

Arumugam, T.V., Phillips, T.M., Cheng, A., Morrell, C.H., Mattson, M.P., Wan, R. (2010). Age and energy intake interact to modify cell stress pathways and stroke outcome. Ann. Neurol. 67, 41-52.

Bastakoty, D., Saraswati, S., Joshi, P., Atkinson, J., Feoktistov, I., Liu, J., Harris, J.L., and Young, P.P. (2016). Temporary, Systemic Inhibition of the WNT/beta-Catenin Pathway promotes Regenerative Cardiac Repair following Myocardial Infarct. Cell Stem Cells Regen Med 2, 10.16966/2472-6990.111.

Bernardo, B.C., Ooi, J.Y.Y., Weeks, K.L., Patterson, N.L., McMullen, J.R. (2018). Understanding Key Mechanisms of Exercise-Induced Cardiac Protection to Mitigate Disease: Current Knowledge and Emerging Concepts. Physiol. Rev. 98, 419-475.

Bockus, L.B., and Humphries, K.M. (2015). cAMP-dependent Protein Kinase (PKA) Signaling Is Impaired in the Diabetic Heart. J Biol Chem 290, 29250-29258.

Bruce-Keller, A.J., Umberger, G., McFall, R., Mattson, M.P. (1999). Food restriction reduces brain damage and improves behavioral outcome following excitotoxic and metabolic insults. Ann. Neurol. 45, 8-15.

Brushia, R.J., and Walsh, D.A. (1999). Phosphorylase kinase: the complexity of its regulation is reflected in the complexity of its structure. Front Biosci 4, D618-641.

Callaghan, N.I. (2016). beta-Adrenergic augmentation of cardiac contractility is dependent on PKA-mediated phosphorylation of myosin-binding protein $\mathrm{C}$ and troponin I. J Physiol 594, 4707-4708.

Castello, L., Maina, M., Testa, G., Cavallini, G., Biasi, F., Donati, A., Leonarduzzi, G., Bergamini, E., Poli, G., Chiarpotto, E. (2011). Alternate-day fasting reverses the ageassociated hypertrophy phenotype in rat heart by influencing the ERK and PI3K signaling pathways. Mech. Ageing Dev. 132, 305-314.

Cerami, E.G., Gross, B.E., Demir, E., Rodchenkov, I., Babur, O., Anwar, N., Schultz, N., Bader, G.D., and Sander, C. (2011). Pathway Commons, a web resource for biological pathway data. Nucleic Acids Res. 39, D685-690.

Cheng, A., Yang, Y., Zhou, Y., Maharana, C., Lu, D., Peng, W., Liu, Y., Wan, R., Marosi, K., Misiak, M., Bohr, V.A., Mattson, M.P. (2016). Mitochondrial SIRT3 Mediates Adaptive Responses of Neurons to Exercise and Metabolic and Excitatory Challenges. Cell Metab. 23, 128-142.

Chowaniec, Z., Skoczyńska, A. (2018). Plasma lipid transfer proteins: The role of PLTP and CETP in atherogenesis. Adv. Clin. Exp. Med. 27, 429-436.

Cox, J., Hein, M.Y., Luber, C.A., Paron, I., Nagaraj, N., and Mann, M. (2014). Accurate proteome-wide label-free quantification by delayed normalization and maximal peptide ratio extraction, termed MaxLFQ. Mol. Cell Proteomics 13, 2513-2526. 
Cox, P.J., Kirk, T., Ashmore, T., Willerton, K., Evans, R., Smith, A., Murray, A.J., Stubbs, B., West, J., McLure, S.W., et al., (2016). Nutritional Ketosis Alters Fuel Preference and Thereby Endurance Performance in Athletes. Cell Metab. 24, 256-268.

Cox, J. and Mann, M. (2012). 1D and 2D annotation enrichment: a statistical method integrating quantitative proteomics with complementary high-throughput data. BMC Bioinformatics. 13 Suppl 16:S12.

Damiola, F., Minh, N. Le., Preitner, N., Kornmann, B., Fleury-Olela, F., Schibler, U. (2000). Restricted feeding uncouples circadian oscillators in peripheral tissues from the central pacemaker in the suprachiasmatic nucleus. Genes \& Development. 14, 2950-2961.

De Cabo, R., and Mattson, M.P. (2019). Impact of intermittent fasting on health, aging and disease. New Engl. J. Med. 381, 2541-2551.

De Lucia, C., Wallner, M., Eaton, D.M., Zhao, H., Houser, S.R., Koch, W.J. (2019). Echocardiographic Strain Analysis for the Early Detection of Left Ventricular Systolic/Diastolic Dysfunction and Dyssynchrony in a Mouse Model of Physiological Aging. J. Gerontol. A. Biol. Sci. Med. Sci. 74, 455-461.

Drake, J.C., Wilson, R.J., Yan, Z. (2016). Molecular mechanisms for mitochondrial adaptation to exercise training in skeletal muscle. FASEB J. 30, 1322.

Ferrario, C.M. (2016). Cardiac remodelling and RAS inhibition. Ther Adv Cardiovasc Dis 10, $162-171$.

Foulquier, S., Daskalopoulos, E.P., Lluri, G., Hermans, K.C.M., Deb, A., Blankesteijn, W.M. (2018). WNT Signaling in Cardiac and Vascular Disease. Pharmacol. Rev. 70, 68-141.

Franklin, M.P., Sathyanarayan, A., and Mashek, D.G. (2017). Acyl-CoA Thioesterase 1 (ACOT1) Regulates PPARalpha to Couple Fatty Acid Flux With Oxidative Capacity During Fasting. Diabetes 66, 2112-2123.

Gannon, N.P., Schnuck, J.K., Vaughan, R.A. (2018). BCAA Metabolism and Insulin Sensitivity -Dysregulated by Metabolic Status? Mol. Nutr. Food Res. 62, e1700756.

Godar, R.J., Ma, X., Liu, H., Murphy, J.T., Weinheimer, C.J., Kovacs, A., Crosby, S.D., Saftig, P., Diwan, A. (2015). Repetitive stimulation of autophagy-lysosome machinery by intermittent fasting preconditions the myocardium to ischemia-reperfusion injury. Autophagy 11, 1537-1560.

Gordon, J.W., Shaw, J.A., Kirshenbaum, L.A. (2011). Multiple facets of NF- $\kappa B$ in the heart: to be or not to NF-kB. Circ. Res. 108, 1122-1132.

Griffioen, K.J., Rothman, S.M., Ladenheim, B., Wan, R., Vranis, N., Hutchison, E., Okun, E., Cadet, J.L., Mattson, M.P. (2013). Dietary energy intake modifies brainstem autonomic dysfunction caused by mutant $\alpha$-synuclein. Neurobiol Aging. 34, 928-935.

Grossmann, K.S., Grund, C., Huelsken, J., Behrend, M., Erdmann, B., Franke, W.W., and Birchmeier, W. (2004). Requirement of plakophilin 2 for heart morphogenesis and cardiac junction formation. J Cell Biol 167, 149-160.

Hara, R., Wan, K., Wakamatsu, H., Aida, R., Moriya, T., Akiyama, M., Shibata, S. (2001). Restricted feeding entrains liver clock without participation of the suprachiasmatic nucleus. Genes Cells. 6, 269-278

Harvie, M.N., Pegington, M., Mattson, M.P., Frystyk, J., Dillon, B., Evans, G., Cuzick, J., Jebb, S.A., Martin, B., Cutler, R.G., et al., (2011). The effects of intermittent or continuous energy restriction on weight loss and metabolic disease risk markers: a randomized trial in young overweight women. Int. J. Obes. (Lond). 35, 714-727.

Harvie, M., Wright, C., Pegington, M., McMullan, D., Mitchell, E., Martin, B., Cutler, R.G., Evans, G., Whiteside, S., Maudsley, S., et al., (2013). The effect of intermittent energy and carbohydrate restriction $\mathrm{v}$. daily energy restriction on weight loss and metabolic disease risk markers in overweight women. Br. J. Nutr. 110, 1534-1547. 
Hers, I., Vincent, E.E., Tavaré, J.M. (2011). Akt signalling in health and disease. Cell Signal. 23, 1515-1527.

Hirth, S., Bühler, A., Bührdel, J.B., Rudeck, S., Dahme, T., Rottbauer, W., Just, S. (2016). Paxillin and Focal Adhesion Kinase (FAK) Regulate Cardiac Contractility in the Zebrafish Heart. PLoS One 11, e0150323.

Hornbeck, P.V., Kornhauser, J.M., Tkachev, S., Zhang, B., Skrzypek, E., Murray, B., Latham, V., and Sullivan, M. (2012). PhosphoSitePlus: a comprehensive resource for investigating the structure and function of experimentally determined post-translational modifications in man and mouse. Nucleic Acids Res 40, D261-270.

Huang da, W., Sherman, B.T., and Lempicki, R.A. (2009). Systematic and integrative analysis of large gene lists using DAVID bioinformatics resources. Nat. Protoc. 4, 4457.

Jagannathan, S., Ogata, Y., Gafken, P.R., Tapscott, S.J., and Bradley, R.K. (2019). Quantitative proteomics reveals key roles for post-transcriptional gene regulation in the molecular pathology of facioscapulohumeral muscular dystrophy. Elife. 8:e41740.

Jessen, N., An, D., Lihn, A.S., Nygren, J., Hirshman, M.F., Thorell, A., and Goodyear, L.J. (2011). Exercise increases TBC1D1 phosphorylation in human skeletal muscle. Am J Physiol Endocrinol Metab 301, E164-171.

Johnson, J.B., Summer, W., Cutler, R.G., Martin, B., Hyun, D.H., Dixit, V.D., Pearson, M., Nassar, M., Telljohann, R., Maudsley, S., et al., (2007). Alternate day calorie restriction improves clinical findings and reduces markers of oxidative stress and inflammation in overweight adults with moderate asthma. Free Radic. Biol. Med. 42, 665-674.

Kashiwaya, Y., Bergman, C., Lee, J.H., Wan, R., King, M.T., Mughal, M.R., Okun, E., Clarke, K., Mattson, M.P., Veech, R.L. (2013). A ketone ester diet exhibits anxiolytic and cognition-sparing properties, and lessens amyloid and tau pathologies in a mouse model of Alzheimer's disease. Neurobiol. Aging. 34, 1530-1539.

Katare, R.G., Kakinuma, Y., Arikawa, M., Yamasaki, F., Sato, T. (2009). Chronic intermittent fasting improves the survival following large myocardial ischemia by activation of BDNF/VEGF/PI3K signaling pathway. J. Mol. Cell. Cardiol. 46, 405-412.

Khuchua, Z., Glukhov, A.I., Strauss, A.W., Javadov, S. (2018). Elucidating the Beneficial Role of PPAR Agonists in Cardiac Diseases. Int. J. Mol. Sci. 19, 3464.

Kim, J., Kang, S.W., Mallilankaraman, K., Baik, S.H., Lim, J.C., Balaganapathy, P., She, D.T., Lok, K.Z., Fann, D.Y., Thambiayah, U, et al., (2018). Transcriptome analysis reveals intermittent fasting-induced genetic changes in ischemic stroke. Hum. Mol. Genet. 27, 1497-1513.

Kim, M.J., Whitehead, N.P., Bible, K.L., Adams, M.E., and Froehner, S.C. (2019). Mice lacking alpha-, beta1- and beta2-syntrophins exhibit diminished function and reduced dystrophin expression in both cardiac and skeletal muscle. Hum Mol Genet 28, 386395.

Kosok, M., Alli-Shaik, A., Bay, B.H., and Gunaratne, J. (2020). Comprehensive Proteomic Characterization Reveals Subclass-Specific Molecular Aberrations within Triplenegative Breast Cancer. iScience 23, 100868.

Kuhn, C., Frank, D., Will, R., Jaschinski, C., Frauen, R., Katus, H.A., and Frey, N. (2009). DYRK1A is a novel negative regulator of cardiomyocyte hypertrophy. J Biol Chem 284, 17320-17327.

Kukreja, R.C., Salloum, F.N., Das, A. (2012). Cyclic guanosine monophosphate signaling and phosphodiesterase-5 inhibitors in cardioprotection. J. Am. Coll. Cardiol. 59,19211927.

Lahey, R., and Khan, S.S. (2018). Trends in Obesity and Risk of Cardiovascular Disease. Curr. Epidemiol. Rep. 5, 243-251. 
Lapek, J.D. Jr., Greninger, P., Morris, R., Amzallag, A., Pruteanu-Malinici, I., Benes, C.H., Haas, W. (2017). Detection of dysregulated protein-association networks by highthroughput proteomics predicts cancer vulnerabilities. Nat. Biotechnol. 35, 983-989.

Lazennec, G., Canaple, L., Saugy, D., and Wahli, W. (2000). Activation of peroxisome proliferator-activated receptors (PPARs) by their ligands and protein kinase A activators. Mol Endocrinol 14, 1962-1975.

Lee, Y., and Kim, E.K. (2013). AMP-activated protein kinase as a key molecular link between metabolism and clockwork. Exp Mol Med 45, e33.

LeWinter, M.M., and Granzier, H.L. (2014). Cardiac titin and heart disease. J Cardiovasc Pharmacol 63, 207-212.

Liehn, E.A., Kanzler, I., Konschalla, S., Kroh, A., Simsekyilmaz, S., Sönmez, T.T., Bucala, R., Bernhagen, J., Weber, C. (2013). Compartmentalized protective and detrimental effects of endogenous macrophage migration-inhibitory factor mediated by CXCR2 in a mouse model of myocardial ischemia/reperfusion. Arterioscler. Thromb. Vasc. Biol. 33, 2180-2186.

Liehn, E.A., Ponomariov, V., Diaconu, R., Streata, I., Ioana, M., Crespo-Avilan, G.E., Hernández-Reséndiz, S., Cabrera-Fuentes, H.A. (2018). Apolipoprotein E in Cardiovascular Diseases: Novel Aspects of an Old-fashioned Enigma. Arch. Med. Res. 49, 522-529.

Lindsey, M.L., Kassiri, Z., Virag, J.A.I., de Castro, Brás, L.E., Scherrer-Crosbie, M. (2018). Guidelines for measuring cardiac physiology in mice. Am. J. Physiol. Heart Circ. Physiol. 314, H733-H752.

Lissandron, V., and Zaccolo, M. (2006). Compartmentalized cAMP/PKA signalling regulates cardiac excitation-contraction coupling. J Muscle Res Cell Motil 27, 399-403.

Liu, D., Zhang, Y., Gharavi, R., Park, H.R., Lee, J., Siddiqui, S., Telljohann, R., Nassar, M.R., Cutler, R.G., Becker, K.G., Mattson, M.P. (2015). The mitochondrial uncoupler DNP triggers brain cell mTOR signaling network reprogramming and CREB pathway up-regulation. J. Neurochem. 134, 677-692.

Liu, Y., Beyer, A., and Aebersold, R. (2016). On the Dependency of Cellular Protein Levels on mRNA Abundance. Cell 165, 535-550.

Liu, Y., Cheng, A., Li, Y.J., Yang, Y., Kishimoto, Y., Zhang, S., Wang, Y., Wan, R., Raefsky, S.M., Lu, D., et al., (2019). SIRT3 mediates hippocampal synaptic adaptations to intermittent fasting and ameliorates deficits in APP mutant mice. Nat. Commun. 10, 1886.

Ma, X., Mani, K., Liu, H., Kovacs, A., Murphy, J.T., Foroughi, L., French, B.A., Weinheimer, C.J., Kraja, A., Benjamin, I.J., et al., (2019). Transcription Factor EB Activation Rescues Advanced $\alpha \mathrm{B}$-Crystallin Mutation-Induced Cardiomyopathy by Normalizing Desmin Localization. J. Am. Heart. Assoc. 8, e010866.

Mager, D.E., Wan, R., Brown, M., Cheng, A., Wareski, P., Abernethy, D.R., Mattson, M.P. (2006). Caloric restriction and intermittent fasting alter spectral measures of heart rate and blood pressurevariability in rats. FASEB J. 20, 631-637.

Makarenko, I., Opitz, C.A., Leake, M.C., Neagoe, C., Kulke, M., Gwathmey, J.K., del Monte, F., Hajjar, R.J., and Linke, W.A. (2004). Passive stiffness changes caused by upregulation of compliant titin isoforms in human dilated cardiomyopathy hearts. Circ Res 95, 708-716.

Marosi, K., Moehl, K., Navas-Enamorado, I., Mitchell, S.J., Zhang, Y., Lehrmann, E., Aon, M.A., Cortassa, S., Becker, K.G., Mattson, M.P. (2018). Metabolic and molecular framework for the enhancement of endurance by intermittent food deprivation. FASEB J. 32, 3844-3858. 
Marosi, K., Kim, S.W., Moehl, K., Scheibye-Knudsen, M., Cheng, A., Cutler, R., Camandola, S., Mattson, M.P. (2016). 3-Hydroxybutyrate regulates energy metabolism and induces BDNF expression in cerebral cortical neurons. J. Neurochem. 139, 769-781.

Mattson, M.P., Longo, V.D., Harvie, M. (2017). Impact of intermittent fasting on health and disease processes. Ageing Res. Rev. 39, 46-58.

Mattson, M.P., and Wan, R. (2005). Beneficial effects of intermittent fasting and caloric restriction on the cardiovascular and cerebrovascular systems. J. Nutr. Biochem. 16, 129-137.

Mattson, M.P., and Arumugam, T.V. (2018). Hallmarks of Brain Aging: Adaptive and Pathological Modification by Metabolic States. Cell Metab. 27, 1176-1199.

Merico, D., Isserlin, R., Stueker, O., Emili, A., and Bader, G.D. (2010). Enrichment map: a network-based method for gene-set enrichment visualization and interpretation. PLoS One 5, el 13984 .

Molkentin, J.D. (2004). Calcineurin-NFAT signaling regulates the cardiac hypertrophic response in coordination with the MAPKs. Cardiovasc Res 63, 467-475.

Mueckler, M. (2001). Insulin resistance and the disruption of Glut4 trafficking in skeletal muscle. J Clin Invest 107, 1211-1213.

Newman, J.C., and Verdin, E. (2017). $\beta$-Hydroxybutyrate: A Signaling Metabolite. Annu. Rev. Nutr. 2017; 37:51-76.

Nepusz, T., Yu, H., and Paccanaro, A. (2012). Detecting overlapping protein complexes in protein-protein interaction networks. Nat. Methods. 9, 471-472.

Pillois, X., Nurden, A.T. (2016). Linkage disequilibrium amongst ITGA2B and ITGB3 gene variants in patients with Glanzmann thrombasthenia confirms that most disease-causing mutations are recent. Br. J. Haematol. 175, 686-695.

Pfeffer, S.R. (2019). NPC intracellular cholesterol transporter 1 (NPC1)-mediated cholesterol export from lysosomes. J. Biol. Chem. 294, 1706-1709.

Plubell, D.L., Wilmarth, P.A., Zhao, Y., Fenton, A.M., Minnier, J., Reddy, A.P., Klimek, J., Yang, X., David, L.L., and Pamir, N. (2017). Extended Multiplexing of Tandem Mass Tags (TMT) Labeling Reveals Age and High Fat Diet Specific Proteome Changes in Mouse Epididymal Adipose Tissue. Mol. Cell. Proteomics. 16, 873-890.

Rappsilber, J., Mann, M., Ishihama, Y. (2007). Protocol for micro-purification, enrichment, pre-fractionation and storage of peptides for proteomics using StageTips. Nat Protoc. 2, 1896-906.

Rengo, G., Parisi, V., Femminella, G.D., Pagano, G., de Lucia, C., Cannavo, A., Liccardo, D., Giallauria, F., Scala, O., Zincarelli, C., et al., (2013). Molecular aspects of the cardioprotective effect of exercise in the elderly. Aging Clin. Exp. Res. 25, 487-497.

Sciarretta, S., Volpe, M., Sadoshima, J. (2014). Mammalian target of rapamycin signaling in cardiac physiology and disease. Circ. Res. 114, 549-564.

Shannon, P., Markiel, A., Ozier, O., Baliga, N.S., Wang, J.T., Ramage, D., Amin, N., Schwikowski, B., and Ideker, T. (2003). Cytoscape: a software environment for integrated models of biomolecular interaction networks. Genome Res. 13, 2498-2504.

Stein, P.K., Soare, A., Meyer, T.E., Cangemi, R., Holloszy, J.O., Fontana, L. (2012). Caloric restriction may reverse age-related autonomic decline in humans. Aging Cell. 11, 644-650.

Stekovic, S., Hofer, S.J., Tripolt, N., Aon, M.A., Royer, P., Pein, L., Stadler, J.T., Pendl, T., Prietl, B., Url, J., Schroeder, S., Tadic, J., Eisenberg, T., Magnes, C., Stumpe, M., Zuegner, E., Bordag, N., Riedl, R., Schmidt, A., Kolesnik, E., Verheyen, N., Springer, A., Madl, T., Sinner, F., de Cabo, R., Kroemer, G., Obermayer-Pietsch, B., Dengjel, J., Sourij, H., Pieber, T.R., Madeo, F. (2019). Alternate Day Fasting Improves 
Physiological and Molecular Markers of Aging in Healthy, Non-obese Humans. Cell Metab. 30, 462-476.

Stokkan, K.A., Yamazaki, S., Tei, H., Sakaki, Y., Menaker, M. (2001). Entrainment of the circadian clock in the liver by feeding. Science 291, 490-493.

Stragier, B., De Bundel, D., Sarre, S., Smolders, I., Vauquelin, G., Dupont, A., Michotte, Y., Vanderheyden, P. (2008). Involvement of insulin-regulated aminopeptidase in the effectsof the renin-angiotensin fragment angiotensin IV: a review. Heart Fail. Rev. 13, 321-337.

Subramanian, A., Tamayo, P., Mootha, V.K., Mukherjee, S., Ebert, B.L., Gillette, M.A., Paulovich, A., Pomeroy, S.L., Golub, T.R., Lander, E.S., et al. (2005). Gene set enrichment analysis: a knowledge-based approach for interpreting genome-wide expression profiles. Proc Natl Acad Sci U S A 102, 15545-15550.

Sutton, E.F., Beyl, R., Early, K.S., Cefalu, W.T., Ravussin, E., Peterson, C.M. (2018). Early Time-Restricted Feeding Improves Insulin Sensitivity, Blood Pressure, and Oxidative Stress Even without Weight Loss in Men with Prediabetes. Cell Metab. 27, 1212-122.

Tyanova, S., Temu, T., and Cox, J. (2016a). The MaxQuant computational platform for mass spectrometry-based shotgun proteomics. Nat. Protoc. 11, 2301-2319.

Tyanova, S., Temu, T., Sinitcyn, P., Carlson, A., Hein, M.Y., Geiger, T., Mann, M., and Cox, J. (2016b). The Perseus computational platform for comprehensive analysis of (prote)omics data. Nat. Methods. 13, 731-740.

Tyrankiewicz, U., Skorka, T., Jablonska, M., Petkow-Dimitrow, P., Chlopicki, S. (2013). Characterization of the Cardiac Response to a Low and High Dose of Dobutamine in the Mouse Model of Dilated Cardiomyopathy by MRI In Vivo, Journal of Magnetic Resonance Imaging 37, 669-677.

Voulgari, C., Pagoni, S., Vinik, A., Poirier, P. (2013). Exercise improves cardiac autonomic function in obesity and diabetes. Metabolism. 62, 609-621.

Wan, R., Ahmet, I., Brown, M., Cheng, A., Kamimura, N., Talan, M., Mattson, M.P. (2010). Cardioprotective effect of intermittent fasting is associated with an elevation of adiponectin levels in rats. J. Nutr. Biochem. 21, 413-417.

Wan, R., Camandola, S., Mattson, M.P. (2003a). Intermittent fasting and dietary supplementation with 2-deoxy-D-glucose improve functional and metabolic cardiovascular risk factors in rats. FASEB J. 17, 1133-1134.

Wan, R., Camandola, S., Mattson, M.P. (2003b). Intermittent food deprivation improves cardiovascular and neuroendocrine responses to stress in rats. J. Nutr. 133, 1921-1929.

Wan, R., Camandola, S., Mattson, M.P. (2004). Dietary supplementation with 2-deoxy-Dglucose improves cardiovascular and neuroendocrine stress adaptation in rats. Am. J. Physiol. Heart Circ. Physiol. 287, H1186-H1193.

Wan, R., Weigand, L.A., Bateman, R., Griffioen, K., Mendelowitz, D., Mattson, M.P. (2014). Evidence that BDNF regulates heart rate by a mechanism involving increased brainstem parasympathetic neuron excitability. J. Neurochem. 129, 573-580.

Weiss, E.P., and Fontana, L. (2011). Caloric restriction: powerful protection for the aging heart and vasculature. Am. J. Physiol. Heart Circ. Physiol. 301, H1205-H1219.

Xiao, K., Sun, J., Kim, J., Rajagopal, S., Zhai, B., Villen, J., Haas, W., Kovacs, J.J., Shukla, A.K., Hara, M.R., et al. (2010). Global phosphorylation analysis of beta-arrestinmediated signaling downstream of a seven transmembrane receptor (7TMR). Proc Natl Acad Sci U S A 107, 15299-15304.

Yang, S., Chen, C., Wang, H., Rao, X., Wang, F., Duan, Q., Chen, F., Long, G., Gong, W., Zou, M.H., Wang, D.W. (2012). Protective effects of Acyl-coA thioesterase 1 on diabetic heart via PPAR $\alpha /$ PGC1 $\alpha$ signaling. PLoS One. 7, e50376. 
Yang, H., Zeng, X.J., Wang, H.X., Zhang, L.K., Dong, X.L., Guo, S., Du, J., Li, H.H., Tang, C.S. (2011). Angiotensin IV protects against angiotensin II-induced cardiac injury via AT4 receptor. Peptides. 32, 2108-2115.

Yavari, A., Bellahcene, M., Bucchi, A., Sirenko, S., Pinter, K., Herring, N., Jung, J.J., Tarasov, K.V., Sharpe, E.J., Wolfien, M., et al., (2017). Mammalian $\gamma 2$ AMPK regulates intrinsic heart rate. Nat. Commun. 8, 1258.

Yu, Z.F., and Mattson, M.P. (1999). Dietary restriction and 2-deoxyglucose administration reduce focal ischemic brain damage and improve behavioral outcome: evidence for a preconditioning mechanism. J. Neurosci. Res. 57, 830-983.

Yudkoff, M., Daikhin, Y., Melo, T.M., Nissim, I., Sonnewald, U., Nissim, I. (2007). The ketogenic diet and brain metabolism of amino acids: relationship to the anticonvulsant effect. Annu. Rev. Nutr. 27, 415-30.

Zablocki, D., and Sadoshima, J. (2013). Angiotensin II and oxidative stress in the failing heart. Antioxid Redox Signal 19, 1095-1109.

Zhang, H.Y., Chu, M.L., Pan, T.C., Sasaki, T., Timpl, R., and Ekblom, P. (1995). Extracellular matrix protein fibulin-2 is expressed in the embryonic endocardial cushion tissue and is a prominent component of valves in adult heart. Dev Biol 167, 18-26.

Zhao, Y., Wang, C., Wang, C., Hong, X., Miao, J., Liao, Y., Zhou, L., and Liu, Y. (2018). An essential role for $\mathrm{Wnt} /$ beta-catenin signaling in mediating hypertensive heart disease. Sci Rep 8, 8996.

Zhou, P., and Pu, W.T. (2016). Recounting Cardiac Cellular Composition. Circ Res 118, 368370. 


\section{FIGURE LEGENDS}

\section{Figure 1. Experimental design}

Fasting blood glucose and ketone levels were measured at the indicated time points. Values are the mean \pm SEM ( $n=20$ mice/group). $* \mathrm{p}<0.05, * * \mathrm{p}<0.01, * * * \mathrm{p}<0.001$ compared to the AL group value. At the 6-month time point, mice were euthanized and hearts were removed and processed for transcriptomic and proteomic analyses. Another set of mice were used for echocardiography experiments ( $n=10$ mice/group).

\section{Figure 2. Impact of intermittent fasting on the heart proteome}

(a) Hierarchical clustering of proteins significantly differentially expressed in each of the IF groups and AL group. The color scale denotes z-score normalized protein abundances. Red denotes higher protein abundance and blue lower protein abundance. (b) Gene ontology-based biological processes and pathways significantly enriched in representative protein clusters across the different groups (FDR < 0.05). Vertical axis denotes protein normalized abundances (red arrow, upregulated; blue arrow, downregulated). (c) Enrichment analysis of modulated processes and pathways in the different IF groups in comparison to the AL group. Each dot represents a functional process or pathway. Positive enrichment score denotes higher protein abundance and negative enrichment score denotes lower protein abundance.

\section{Figure 3. Comparison of transcriptome and proteome rewiring during IF}

(a) Comparison of proteome and transcriptome abundances in AL and different IF groups. Protein levels are represented by average log-transformed normalized abundances and transcriptome levels are represented using average log-transformed FPKM within each group (b) Functional processes and pathways altered by changes in transcriptome and proteome abundances through different IF regimens are plotted. (Benjamini-Hochberg FDR < 0.05). Each dot on the plot represents a modulation in pathway or process. The scores are indicative of abundance changes of transcriptome or proteome levels over AL. Upregulation and downregulation of components are denoted by positive and negative scores, respectively. (c) Heat map shows changes in the levels of transcripts and proteins associated with fatty acid oxidation in EOD compared to AL group. Abundance levels are marked by red indicating maximum and blue indicating minimum. (d) EOD induced changes in mRNA and protein abundances of enzymes are visualized as a scatter plot. 


\section{Figure 4. Differential effects of IF regimen on functional transition networks of the heart} (a-c) Functional remodeling of heart proteome in response to IF12 (a), IF16 (b) and EOD (c) visualized as a functional transition network. GSEA for biological processes and pathways performed for each IF regimen comparison is shown as an enrichment map. Functions significantly $(\mathrm{p}<0.01)$ enriched among the upregulated and downregulated proteins for each comparison are indicated by red and blue nodes, respectively. The size of the nodes indicates the number of proteins within the node, and edges link nodes that share common proteins.

\section{Figure 5. Network mapping of IF-responsive proteins}

(a) Proteins that display significantly altered abundance in response to IF are overlaid on consensus protein-protein interaction network constructed using all IF-responsive proteins. The proteins that sequentially show differential modulation are indicated with different colors as yellow (IF12), light brown (IF16) and dark brown (EOD). (b and c) The top densely connected functional protein clusters associated with fatty acid oxidation and BCCA metabolism (b) and lipid metabolism (c) are shown. The color of each node indicates the IF regimen for which the protein was differentially expressed. Yellow, light brown and dark brown indicate IF12, IF16 and EOD regimens, respectively. Node border color represents protein expression status in the extended EOD fasting group, with red denoting increased expression and blue denoting decreased expression, compared to AL group. The heat maps show proteome changes for the proteins in the respective clusters. Red denotes increased expression and blue denotes decreased expression.

\section{Figure 6. Proteomic analysis reveals metabolic pathways in the heart impacted by intermittent fasting}

(a) Proteins with altered functional network associations (colored nodes) are shown against a background protein association network assembled from positive and negative co-regulation analysis of all quantified proteins. Only those protein co-regulations that were significant (adjusted $\mathrm{p}<0.01$ ) were included in the background protein association network. Functional involvement of perturbed proteins across different IF regimens is shown below. (b) Association network of mitochondrial proteins impacted by IF in the heart. The perturbed proteins specific to each IF or AL regimen are indicated by yellow (AL), green (IF12), blue (IF16) and pink (EOD) nodes. Perturbations inferred from positive associations are indicated as red edges and those from negative associations are shown as blue edges. All gray nodes 
denote proteins sharing association perturbation with the indicated mitochondrial proteins. Colored borders around the nodes are representative of pathways or processes.

\section{Figure 7. Phosphoproteome landscape of IF-induced changes in heart}

(a) Phosphorylation sites showing significant changes in the hearts of mice in the IF12, IF16 and EOD are shown as volcano plots along with functional annotations of their respective phosphoproteins. (b) Pathways significantly enriched ( $\mathrm{p}$ value $<0.05$ ) among the differentially altered phosphoproteins across the three IF regimens are visualized as a heatmap. The color scale represents negative log10-transformed p values. Enrichment among phosphoproteins with increased phosphorylations is shown in red and those with decreased phosphorylations is shown in blue. (c) Representative immunoblots and quantification illustrating cGMP-PKG pathway activation in IF16 compared to AL. $* \mathrm{P}<0.05$ compared with AL.

\section{Figure 8. Site-specific alteration of IF-responsive phosphoproteins}

(a) Hierarchical clustering of differentially altered phosphopeptides showing increased (red) and decreased (blue) phosphosite abundance. Kinase motifs enriched $(\mathrm{p}<0.05)$ in representative clusters are shown. (b-e) Temporal alteration of specific IF-responsive phosphorylation sites in insulin signaling (a), PPAR signaling (b), cholesterol metabolism (c), and heart structural remodeling (d) are visualized as heat maps. Red indicates increased phosphosite abundance and blue denotes decreased phosphosite abundance. The overall trend among the diet groups based on phosphoproteome landscape is indicated as brown triangles.

\section{Figure 9. Echocardiographic analyses reveal beneficial effects of intermittent fasting on cardiac stress adaptation}

(a) Dobutamine stress tests suggest that IF enhances the ability of the heart to adapt to stress. Heart rate and ejection fraction following administration of dobutamine were significantly greater in hearts of IF16 mice compared to AL mice. Values are the mean \pm SD; $n=10$ each; *p $<0.05$. Representative images of long axis M-Mode raw data before and after dobutamineinduced stress are shown in the right panel. (b) Increased contractility reserve in hearts of IF16 mice compared with AL mice as illustrated by global longitudinal strain (GLS) (upper panel), and radial strain (lower panel). Values are the mean \pm SD ( $n=10$ each; **p $<0.01$, $* * * \mathrm{p}<0.001)$ before and after dobutamine (D) administration. Representative images of 
myocardial movement (upper panel) and radial strain (lower panel) before and after dobutamine-induced stress.

\section{Figure 10: Time-resolved pathway modulation summary map in heart during IF}

Changes in abundance of proteins and phosphorylation sites with different IF regimens visualized in the context of cellular signaling pathways (assembled from KEGG pathway database). Multiple unique phosphorylation sites on proteins are mapped using filled colored circles sequentially decked on top of proteins. For proteins marked with asterisk only selected phosphorylation sites among many modulated sites are visualized. Abundance changes of proteins are visualized using filled rectangular panels and changes in multiple isoforms of proteins are also indicated within each node. Circle/rectangle in red or blue denote significant increase or decrease in phosphorylation site/protein abundance, respectively, and white circle/rectangle indicates no significant change. Arrows with pointed head correspond to activation and those with blunted head denote inactivation within pathways. Protein nodes highlighted in green represent kinases predicted to be modulated with IF. 


\section{BLOOD MEASUREMENTS}

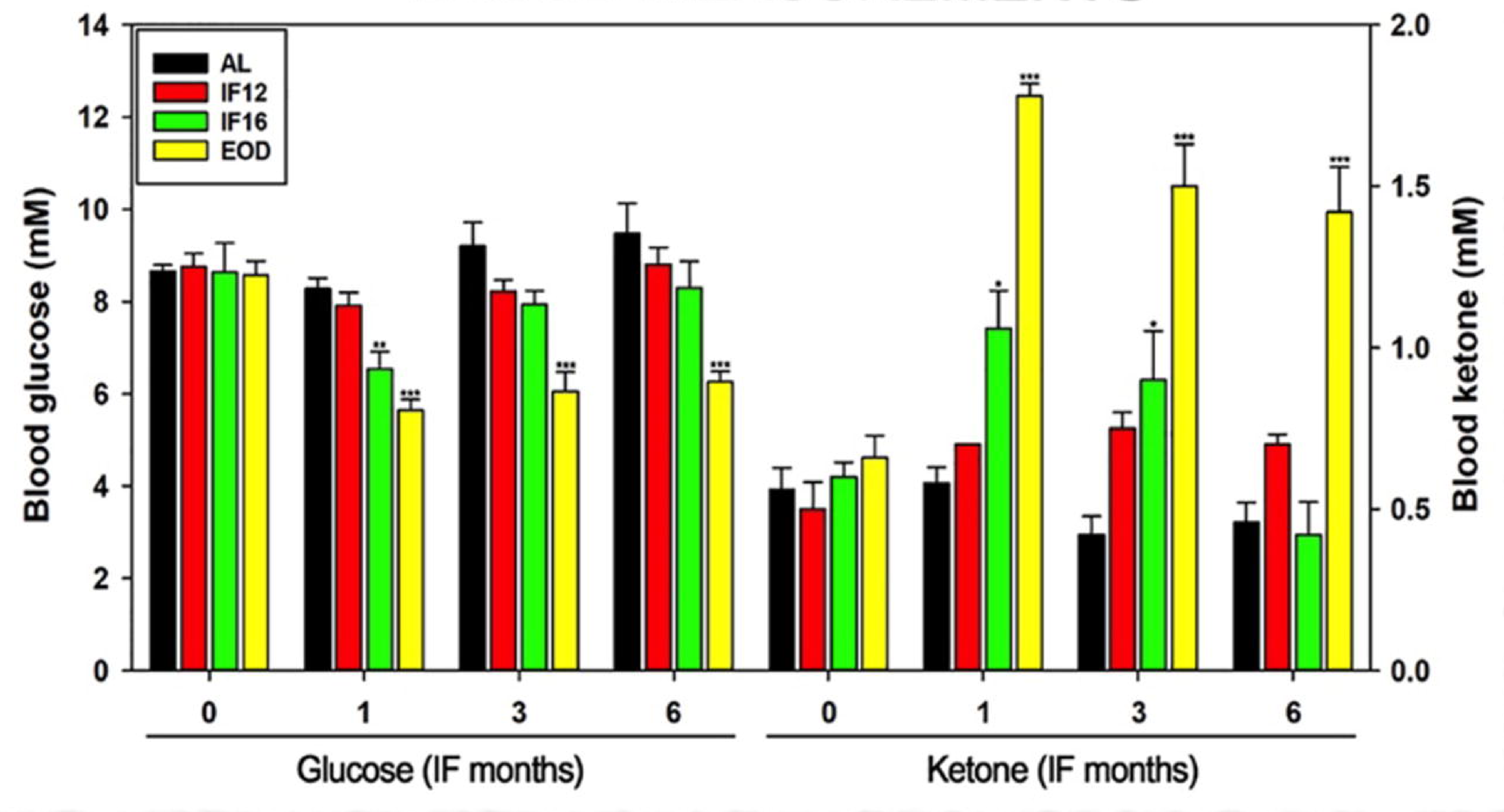

\section{Transcriptomics}

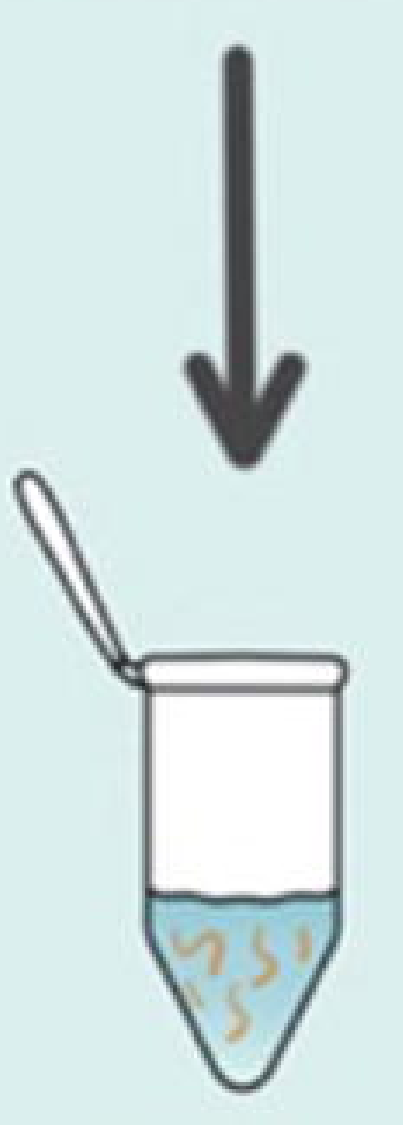

mRNA

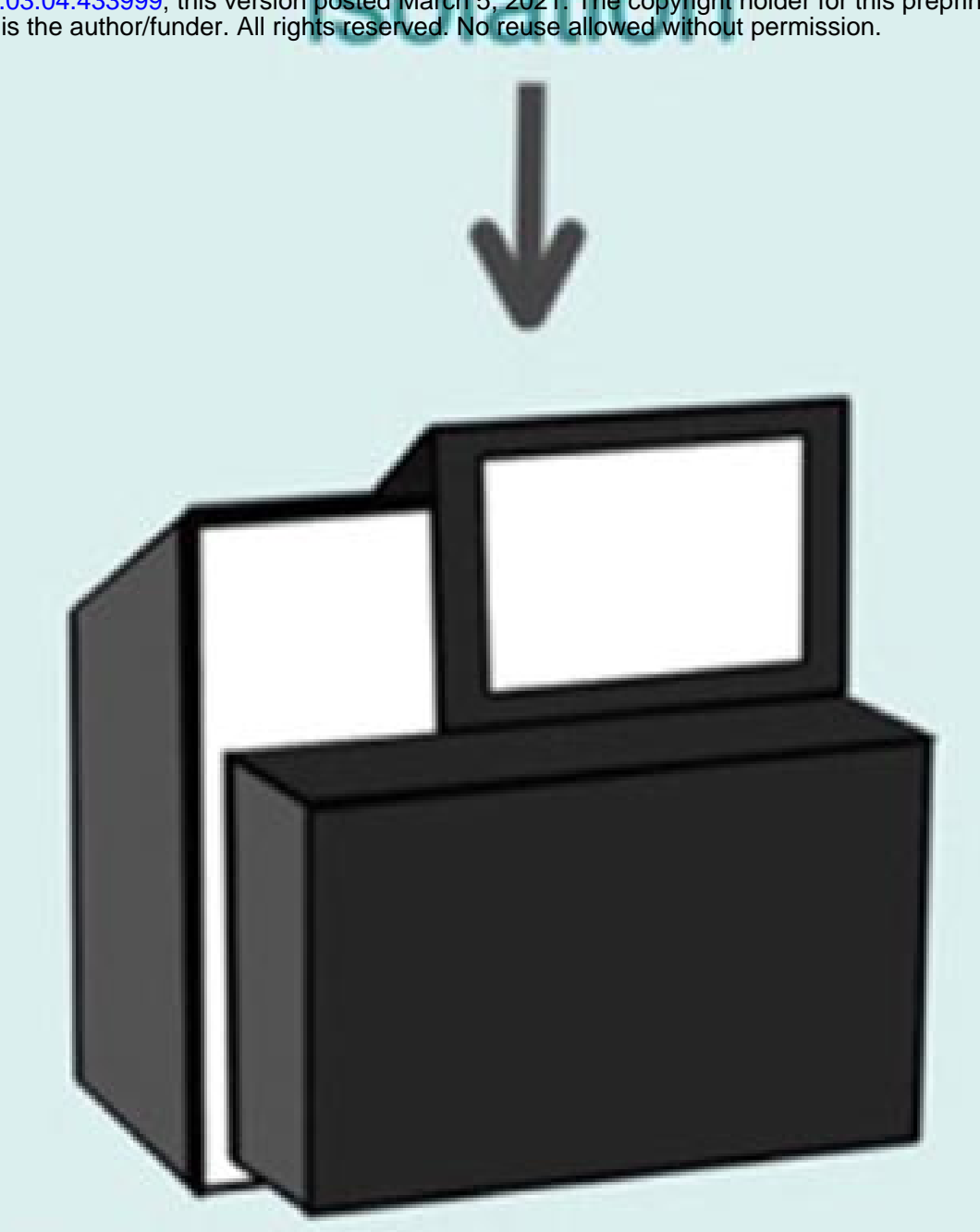

RNA Sequencing

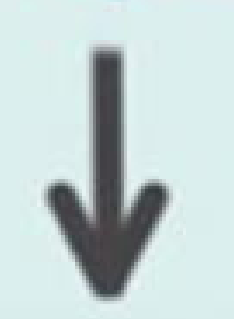

Differentially expressed genes
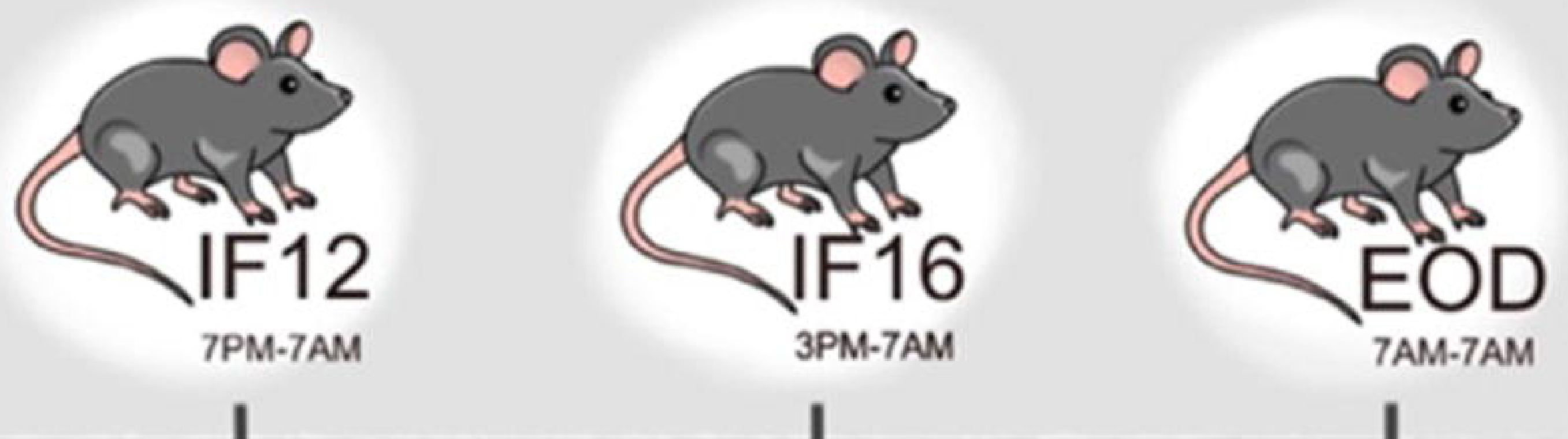

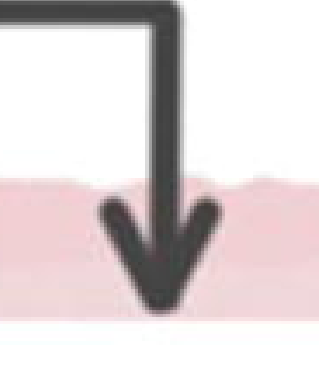

Proteomics

\section{Heart Function}

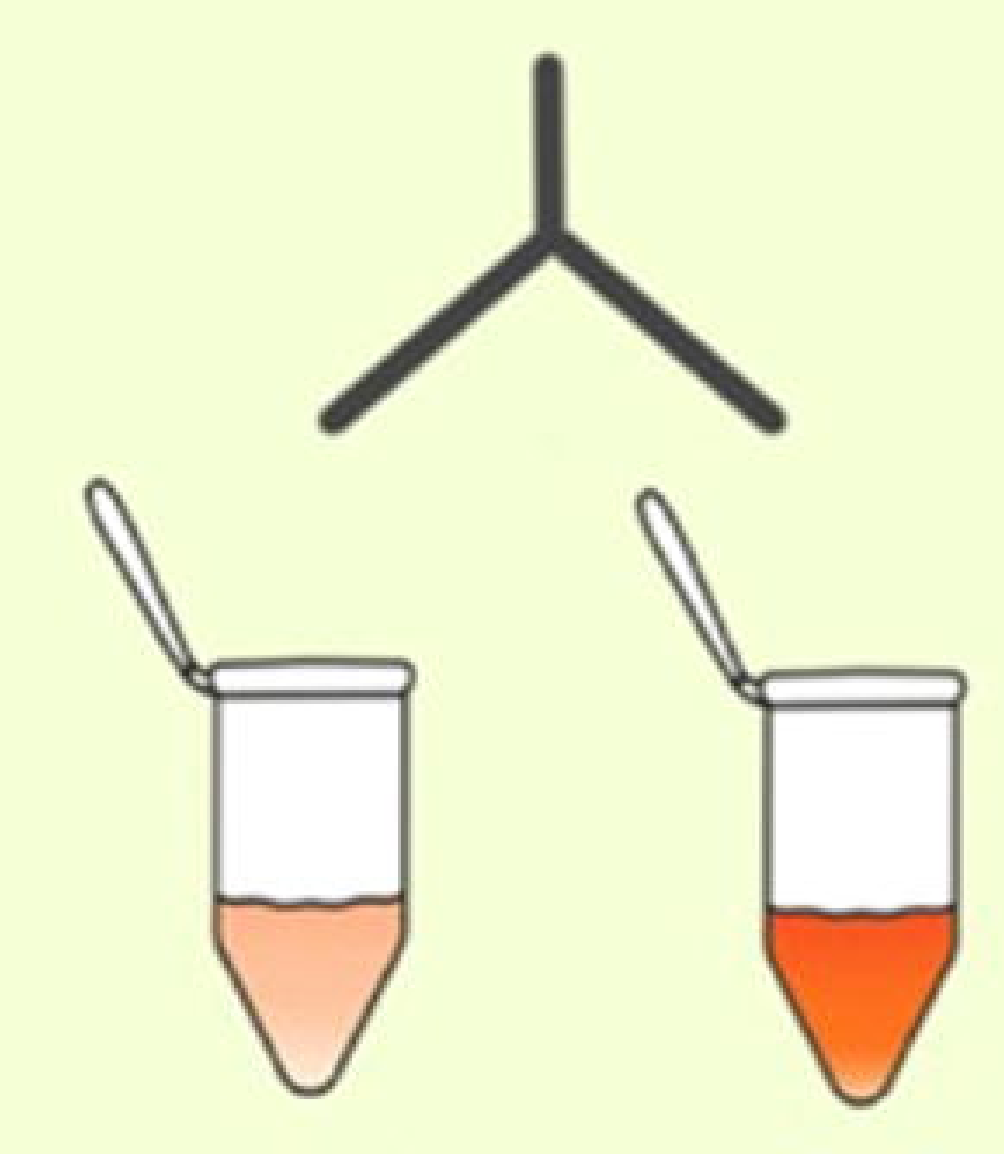

Lysate

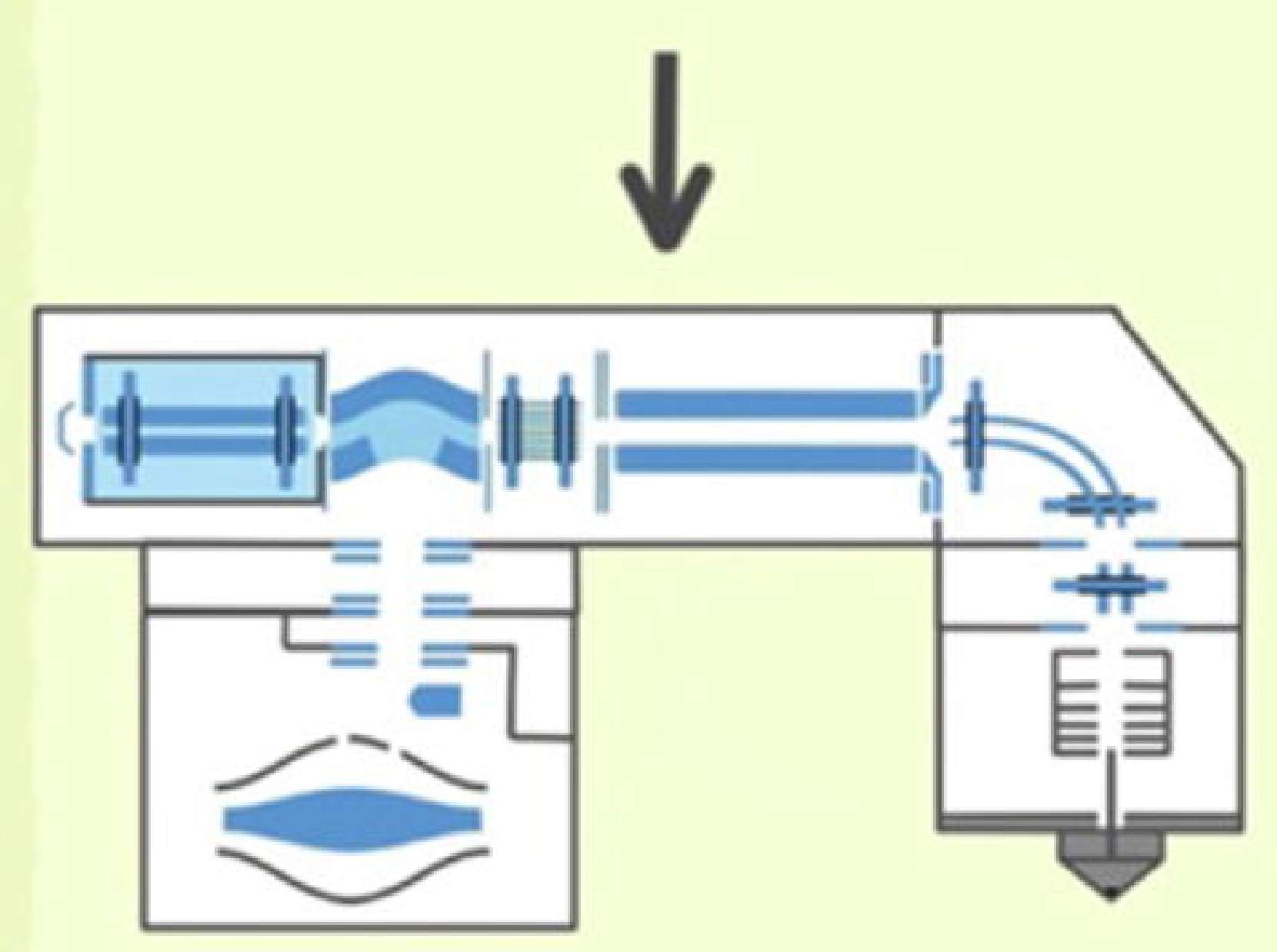

LC-MS proteome analysis

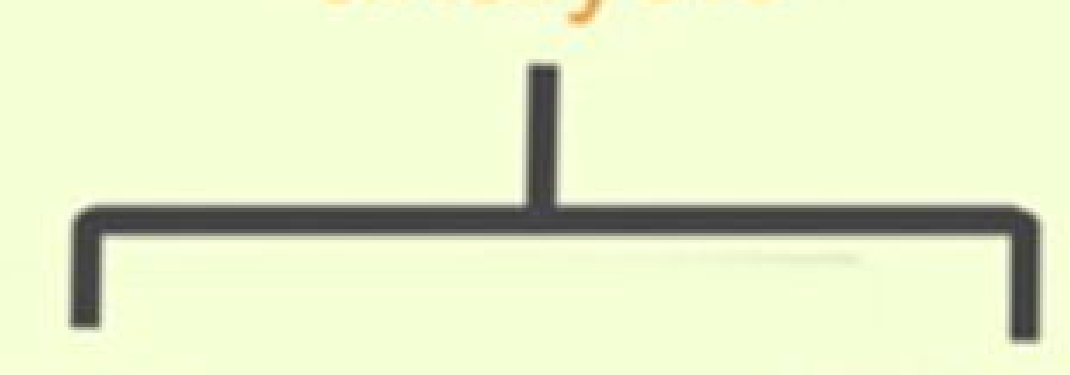

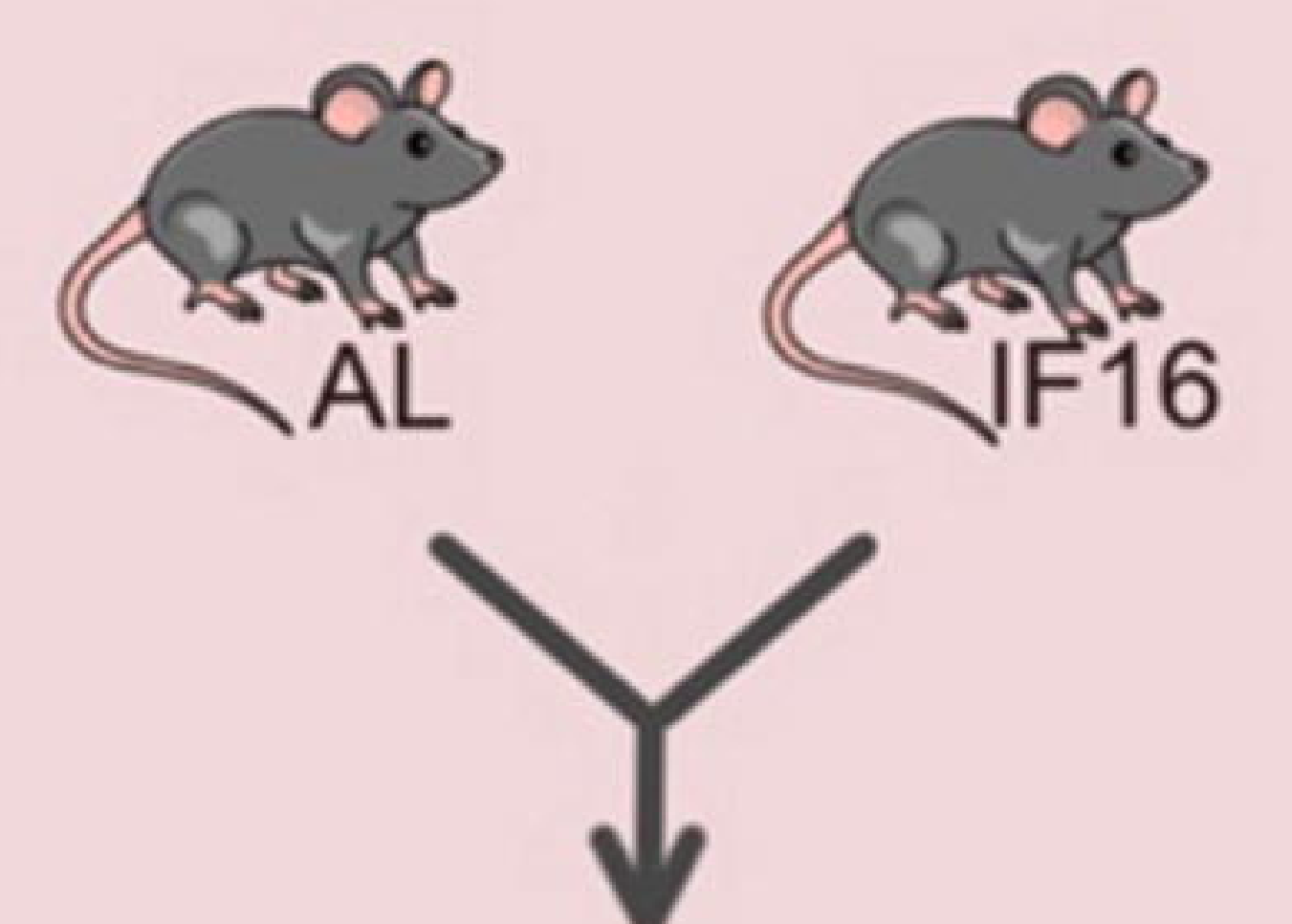

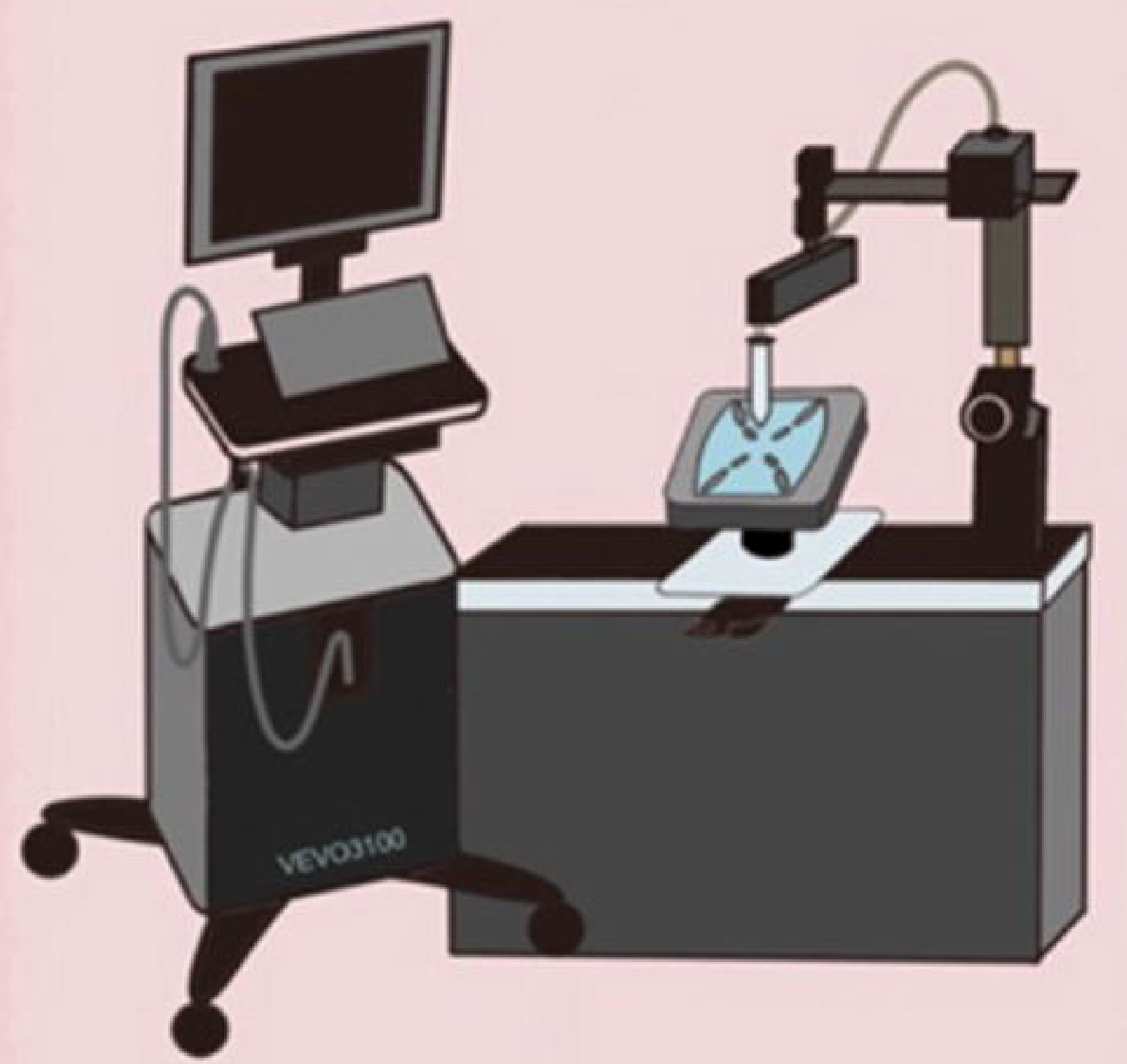

Echocardiography

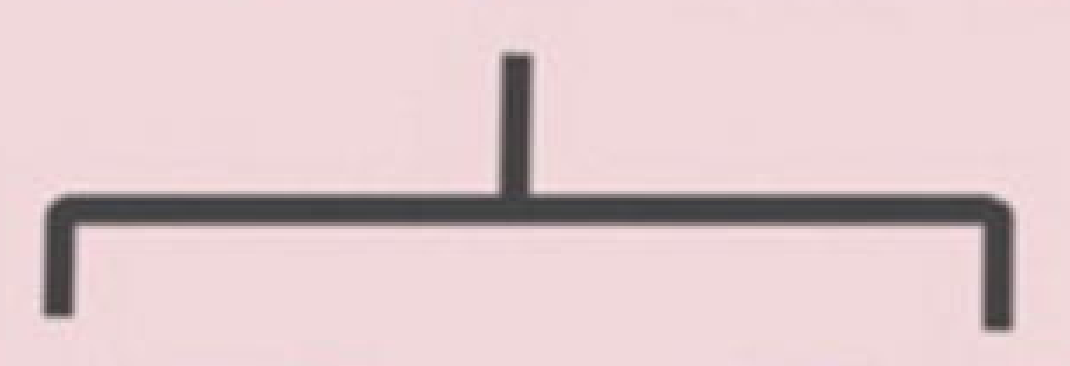

Myocardial Cardiac function dynamics 

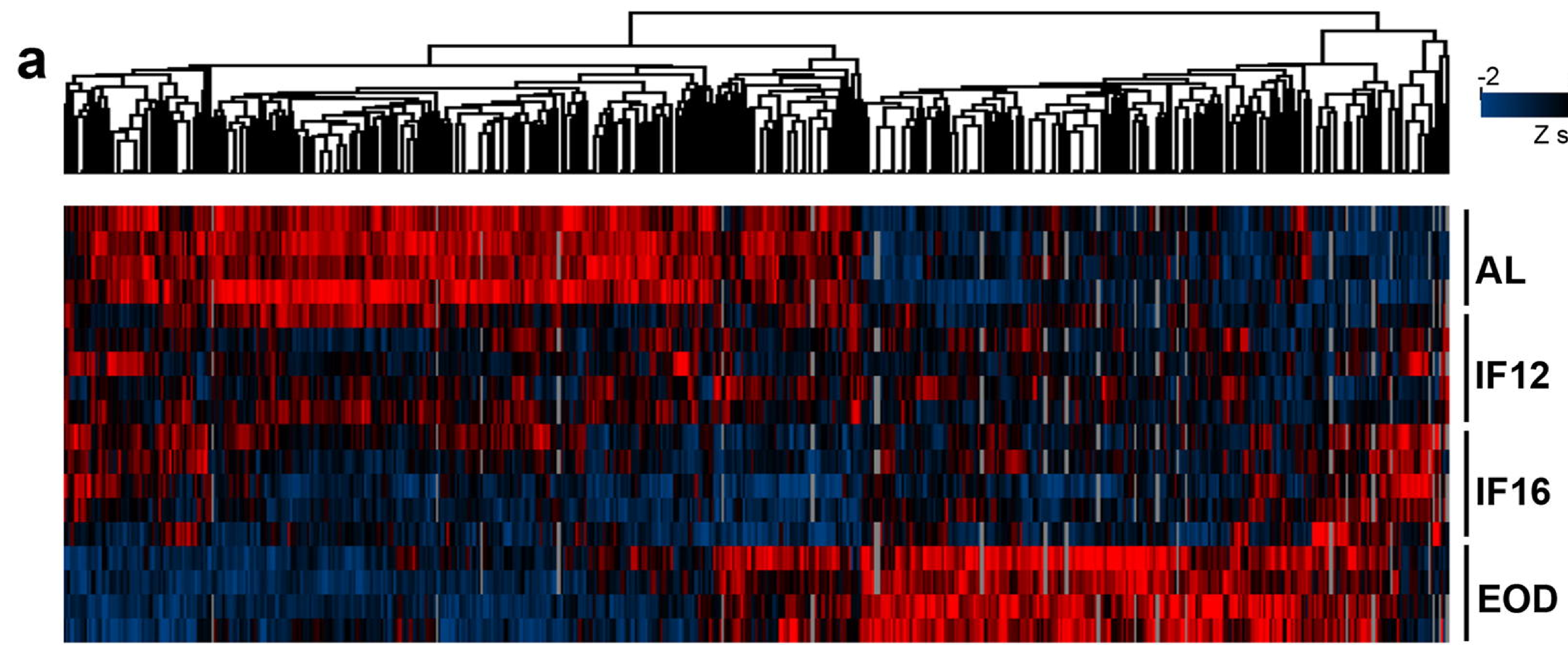

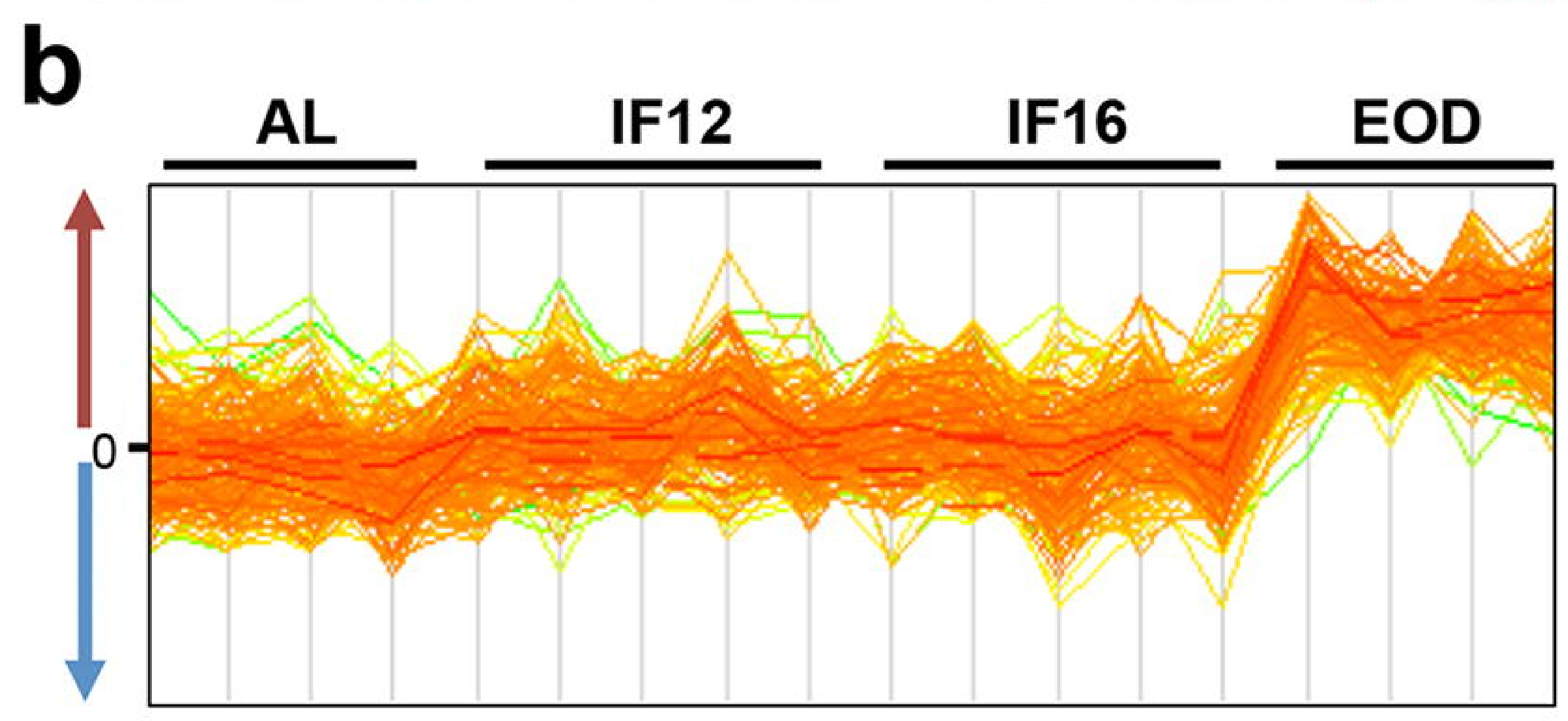

RNA metabolic process, mRNA processing,

Gene expression, transcription, RNA splicing

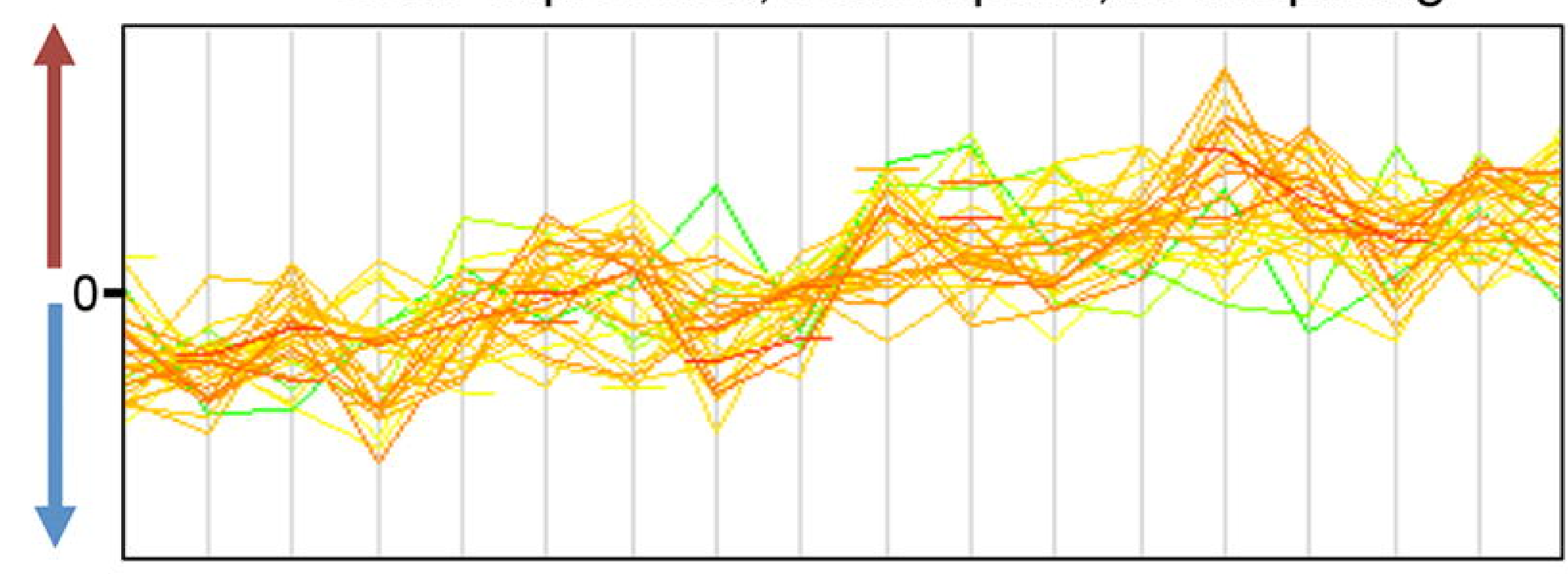

Integral to membrane

C

IF12 vs AL

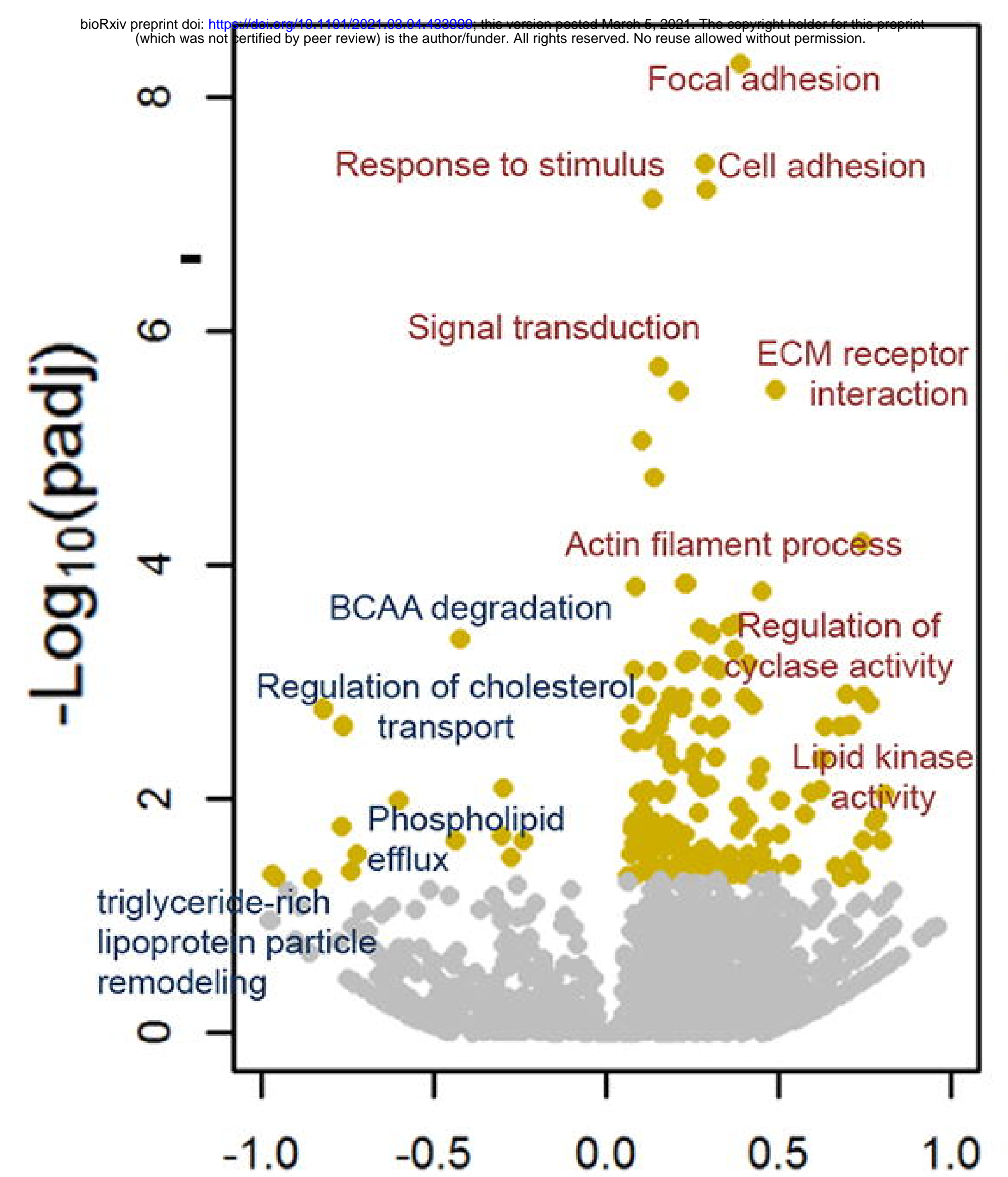

Enrichment Score

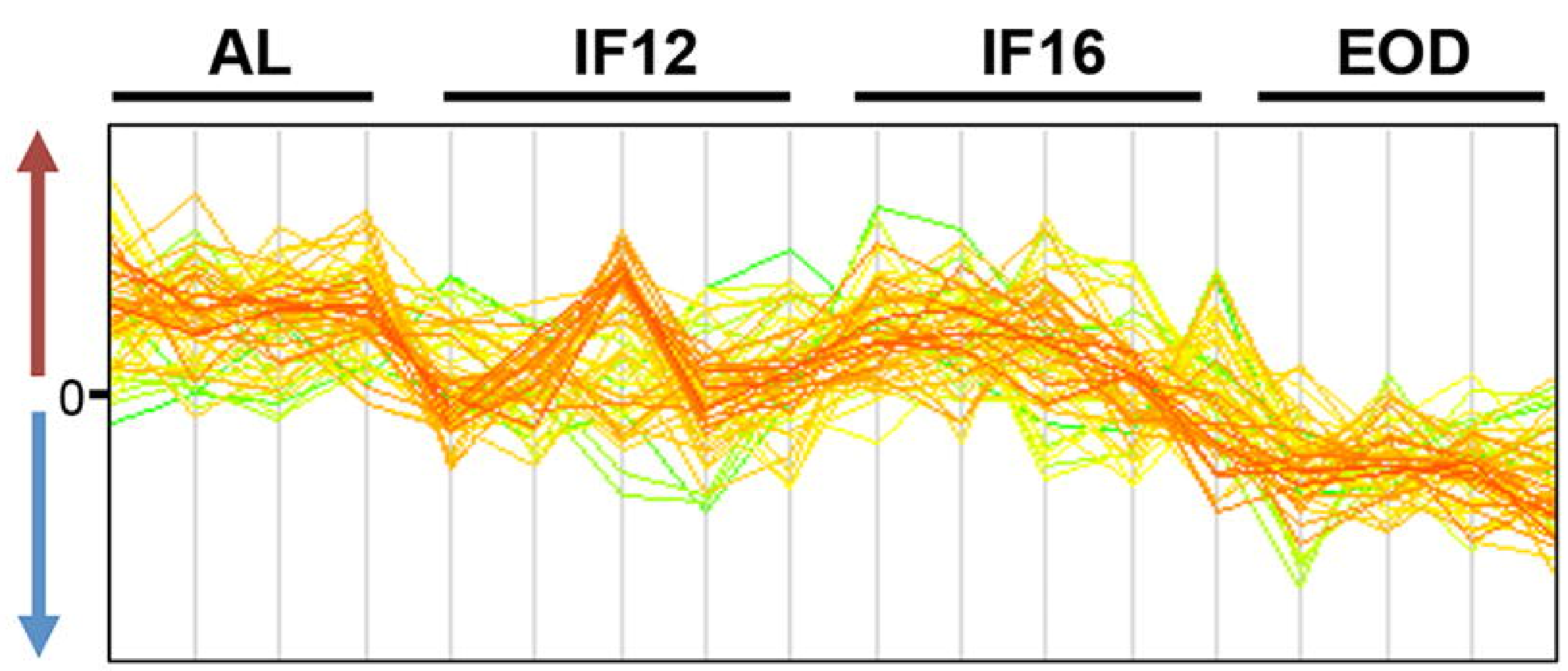

Lipid/cholesterol transport, triglyceriderich protein particle, phospholipid efflux

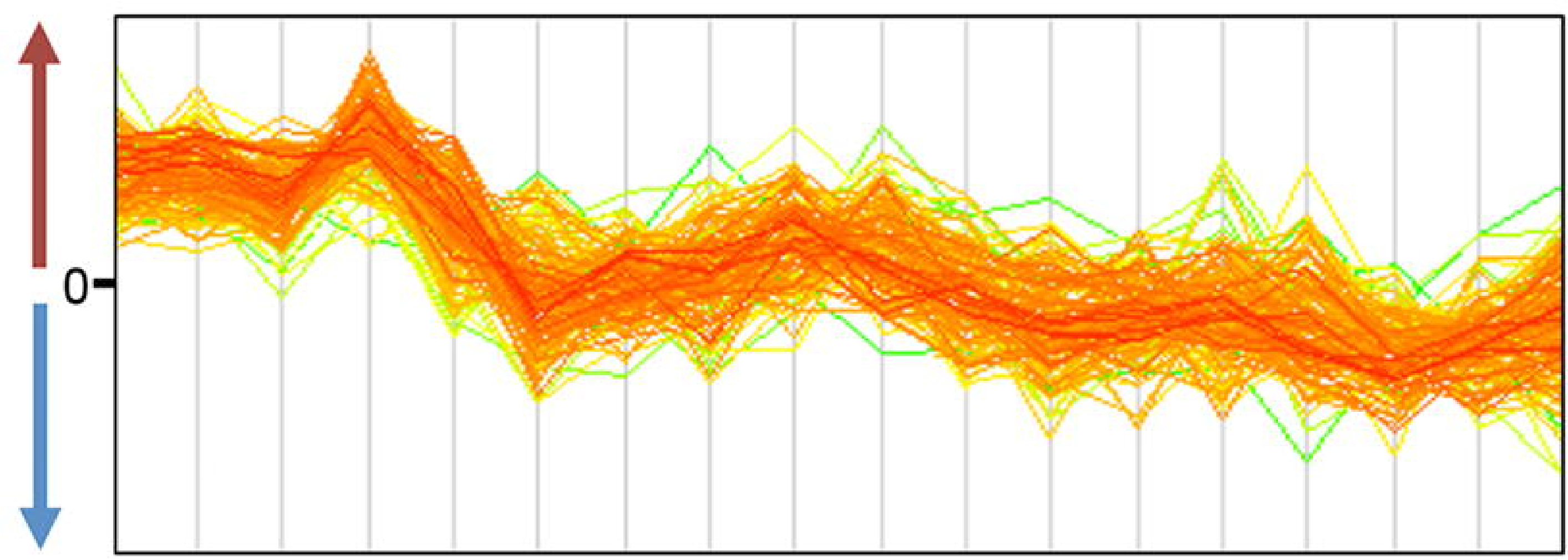

Mitochondrion, catalytic activity, oxoacid metabolic process, ketone metabolic process

Figure 2

IF16 vs $\mathrm{AL}$

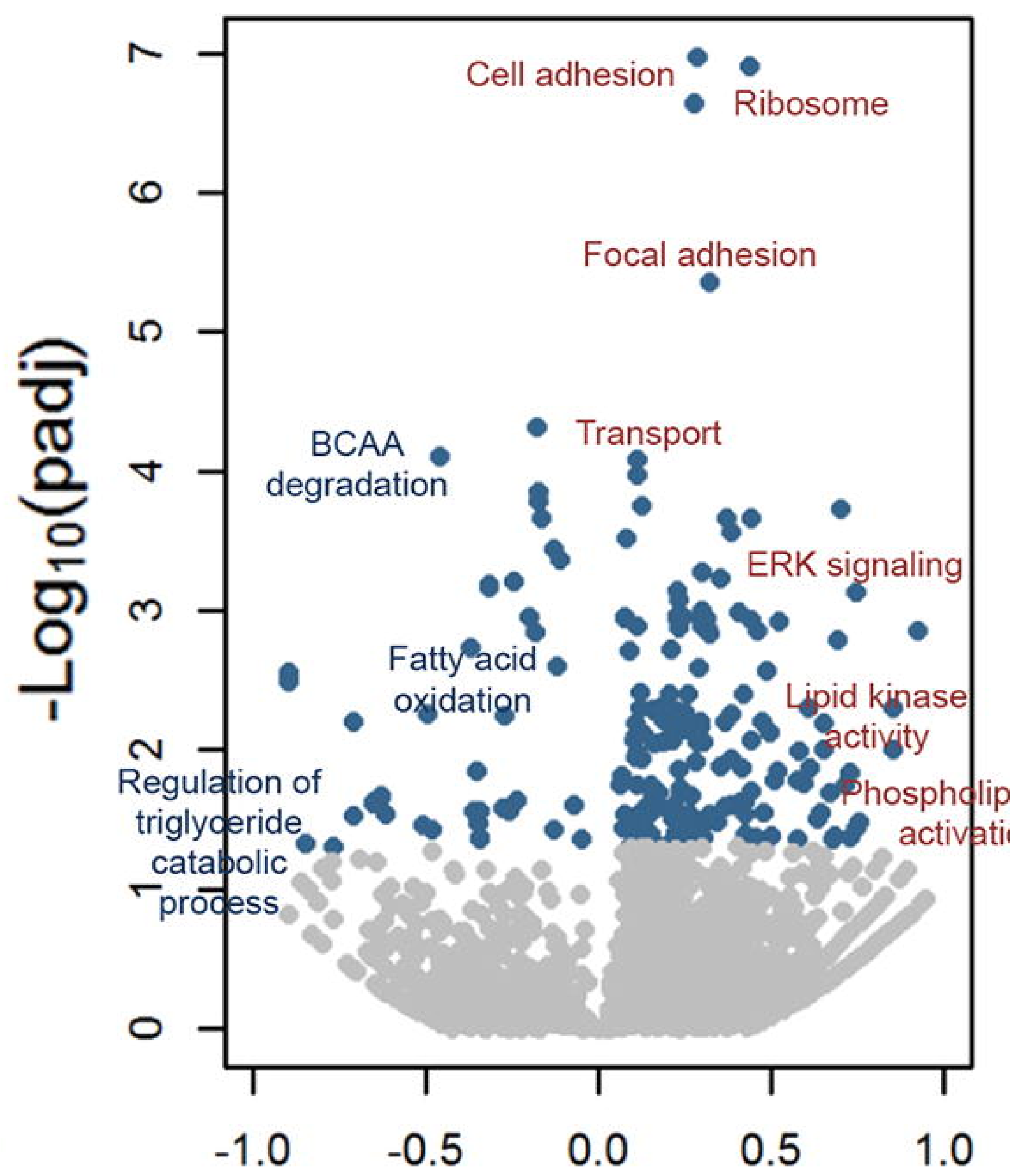

Enrichment Score
EOD vs $A L$

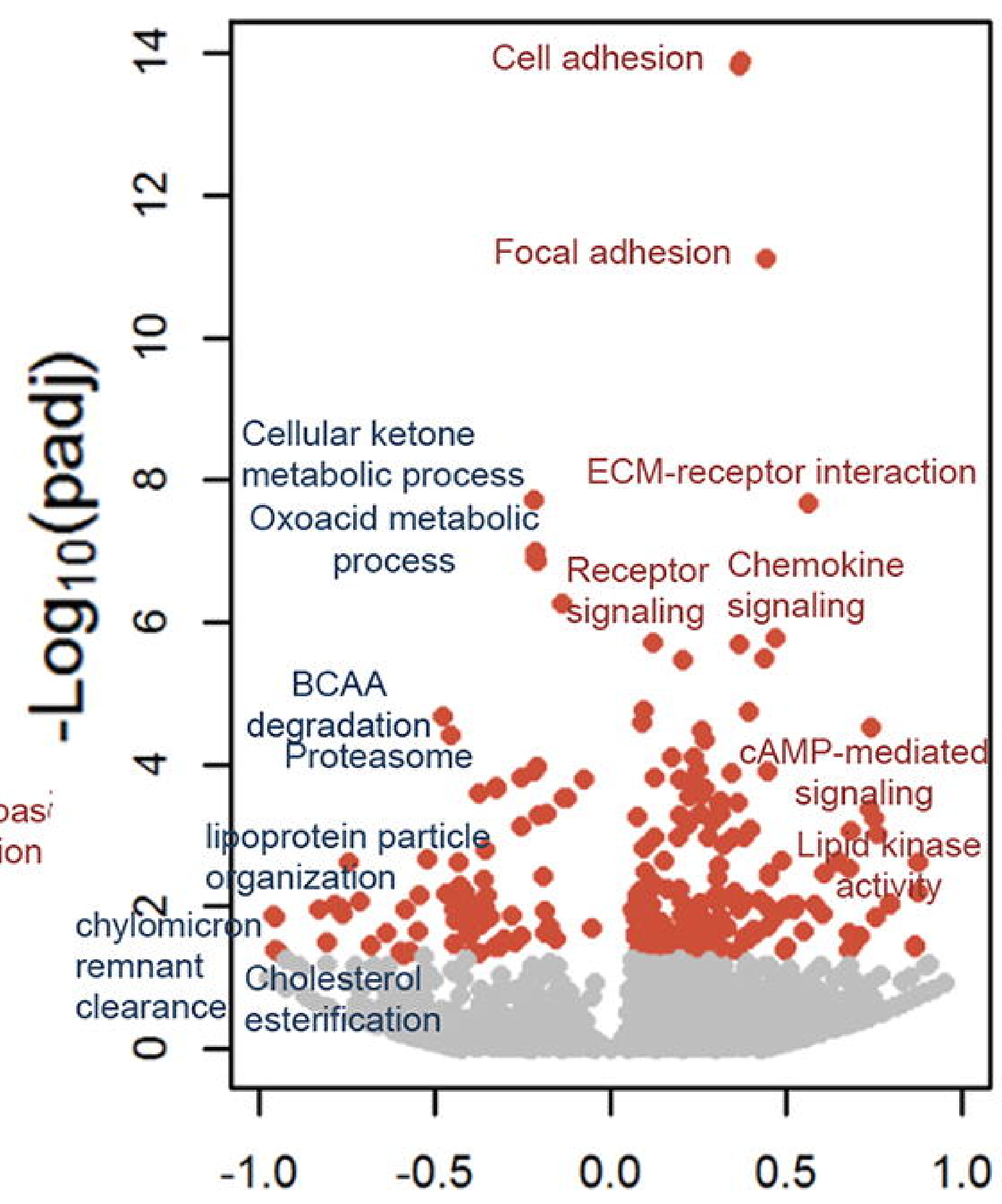

Enrichment Score 
AL

6374 overlaps

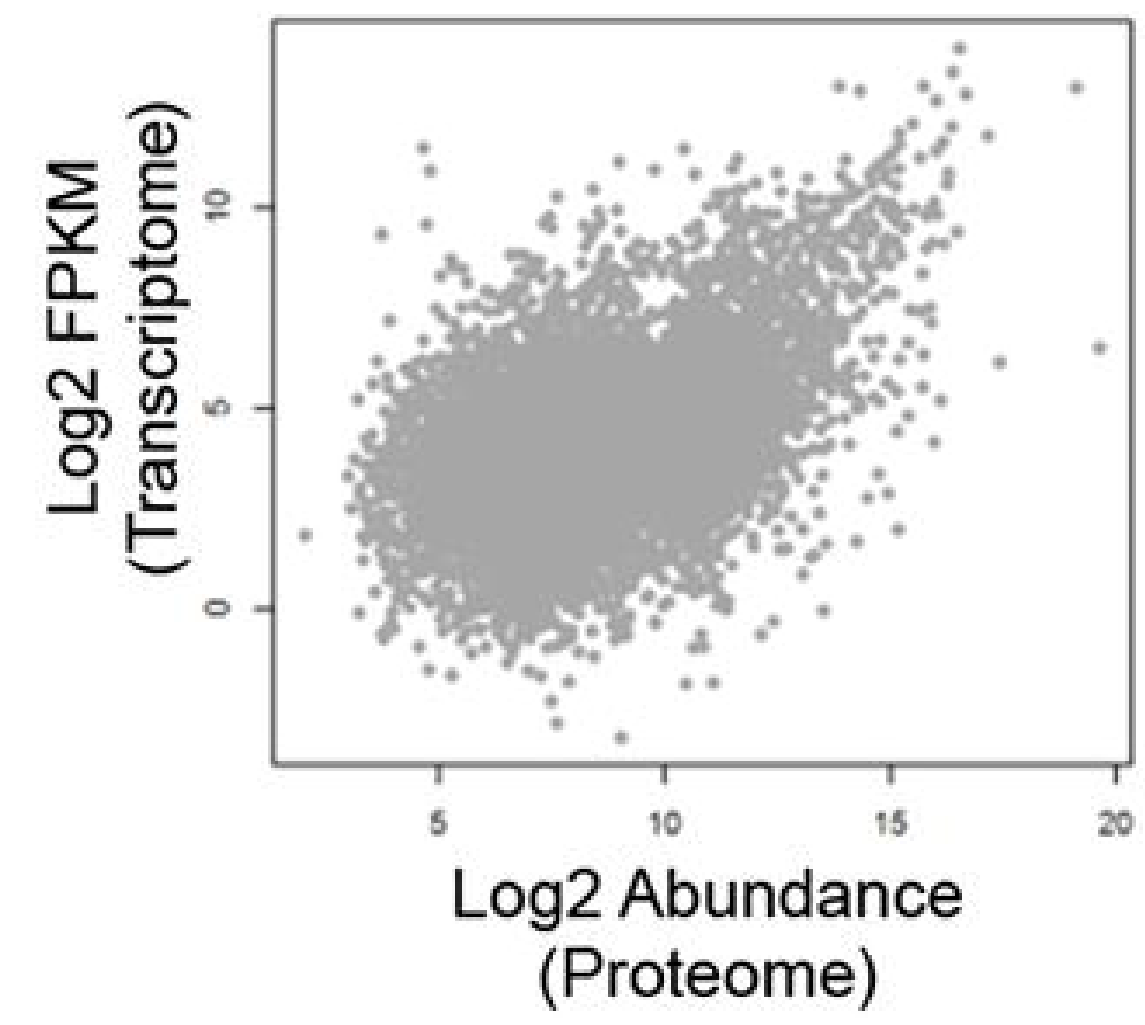

IF16

6370 overlaps

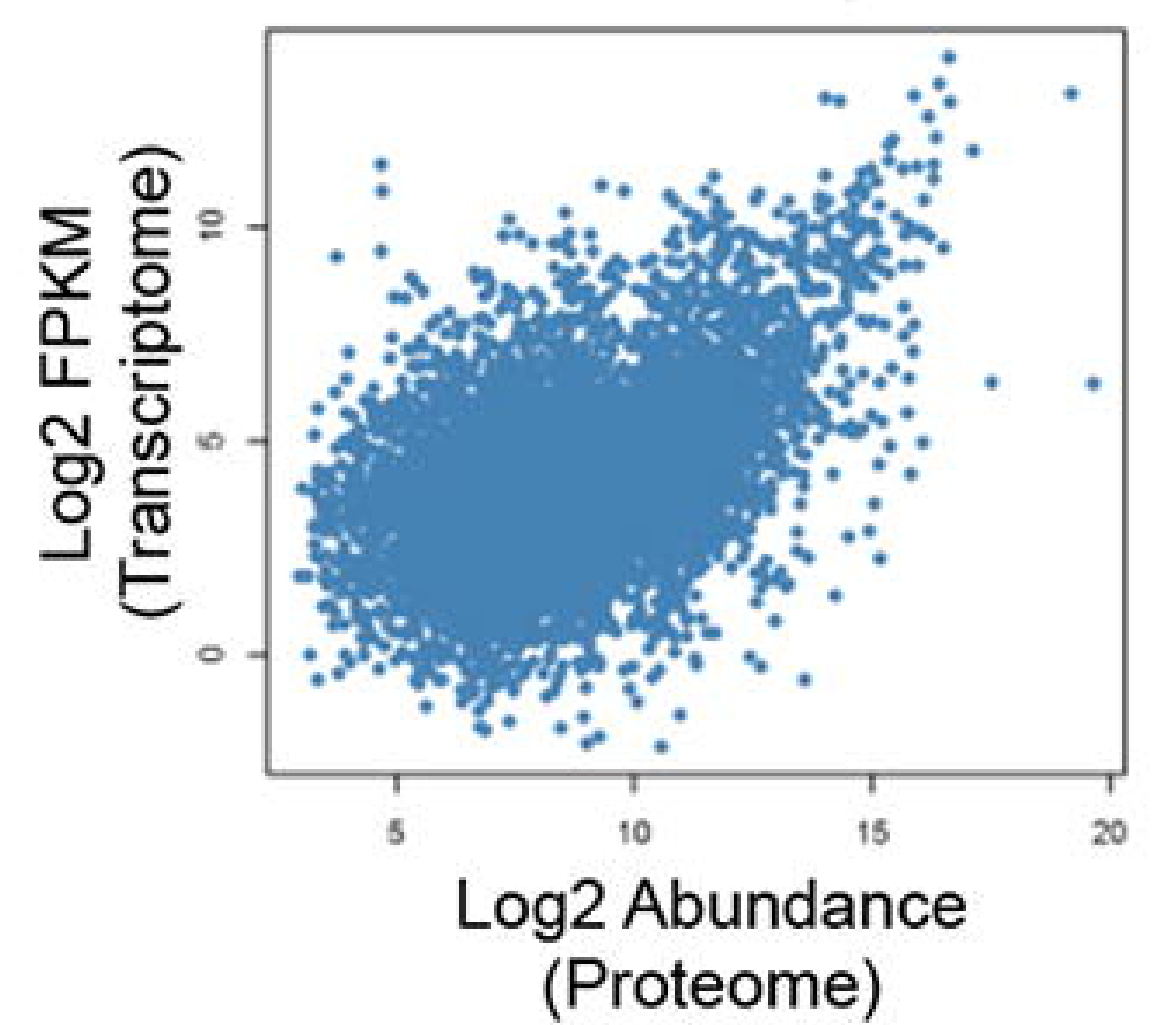

IF12

6394 overlaps

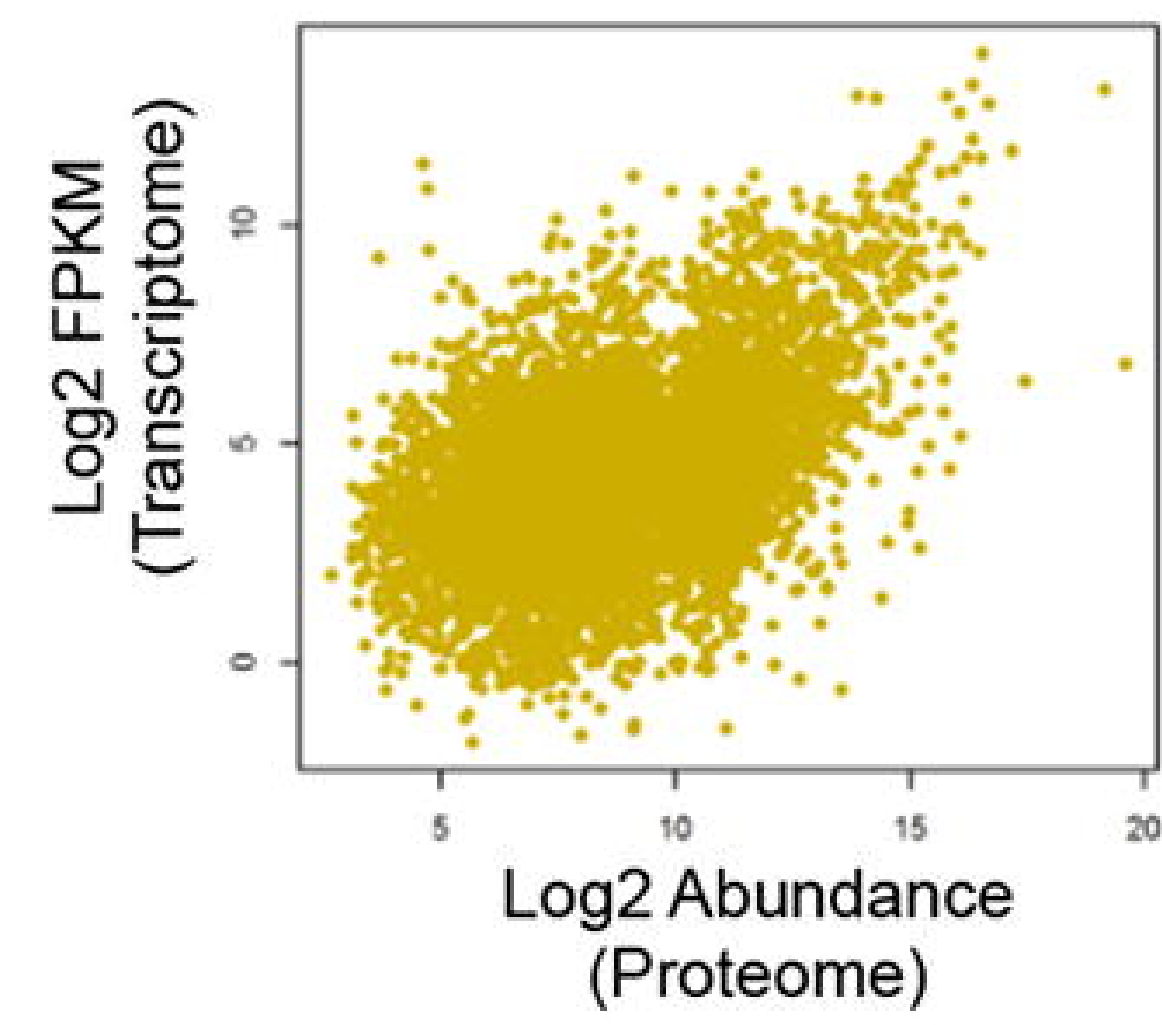

EOD

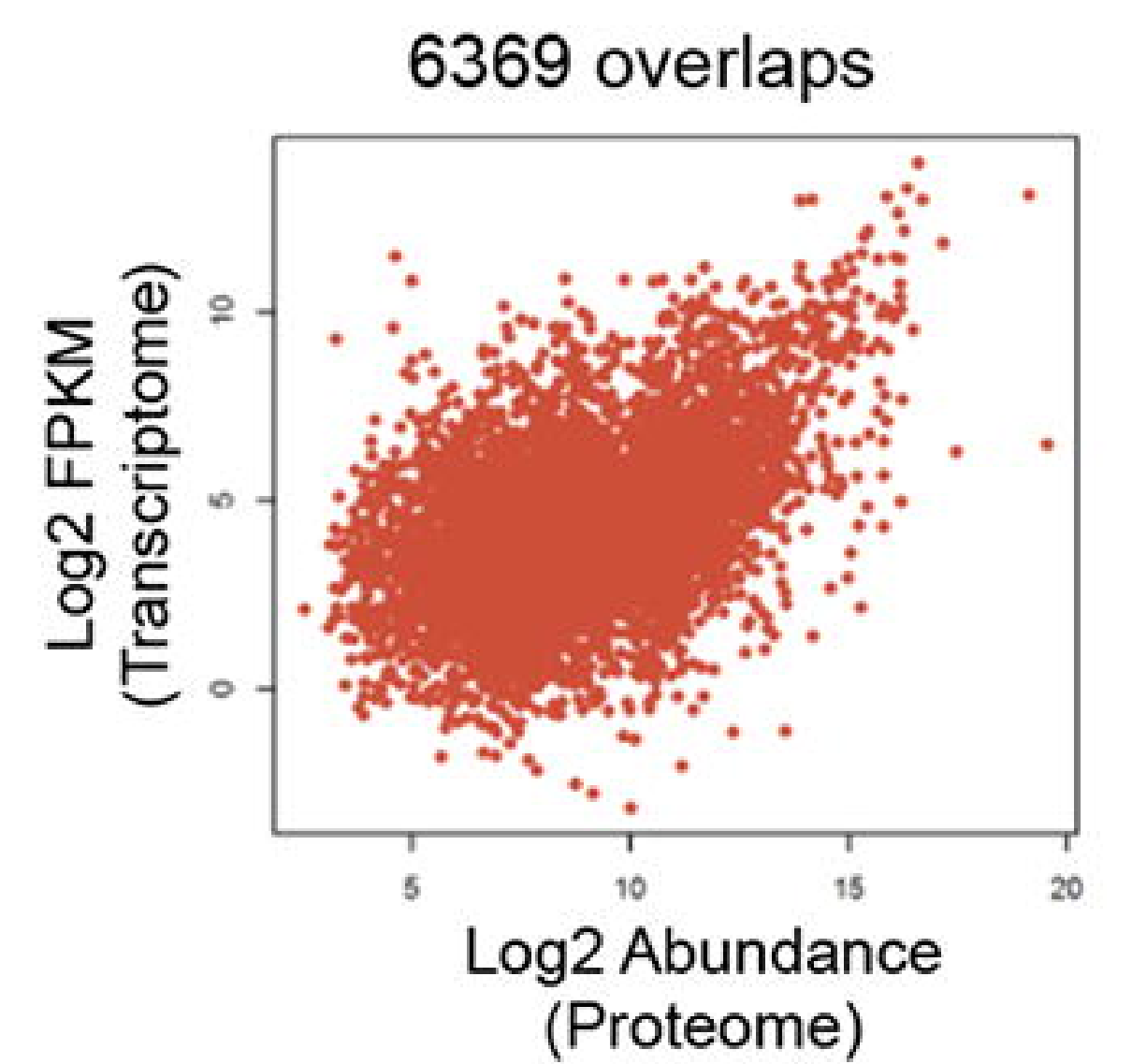

C

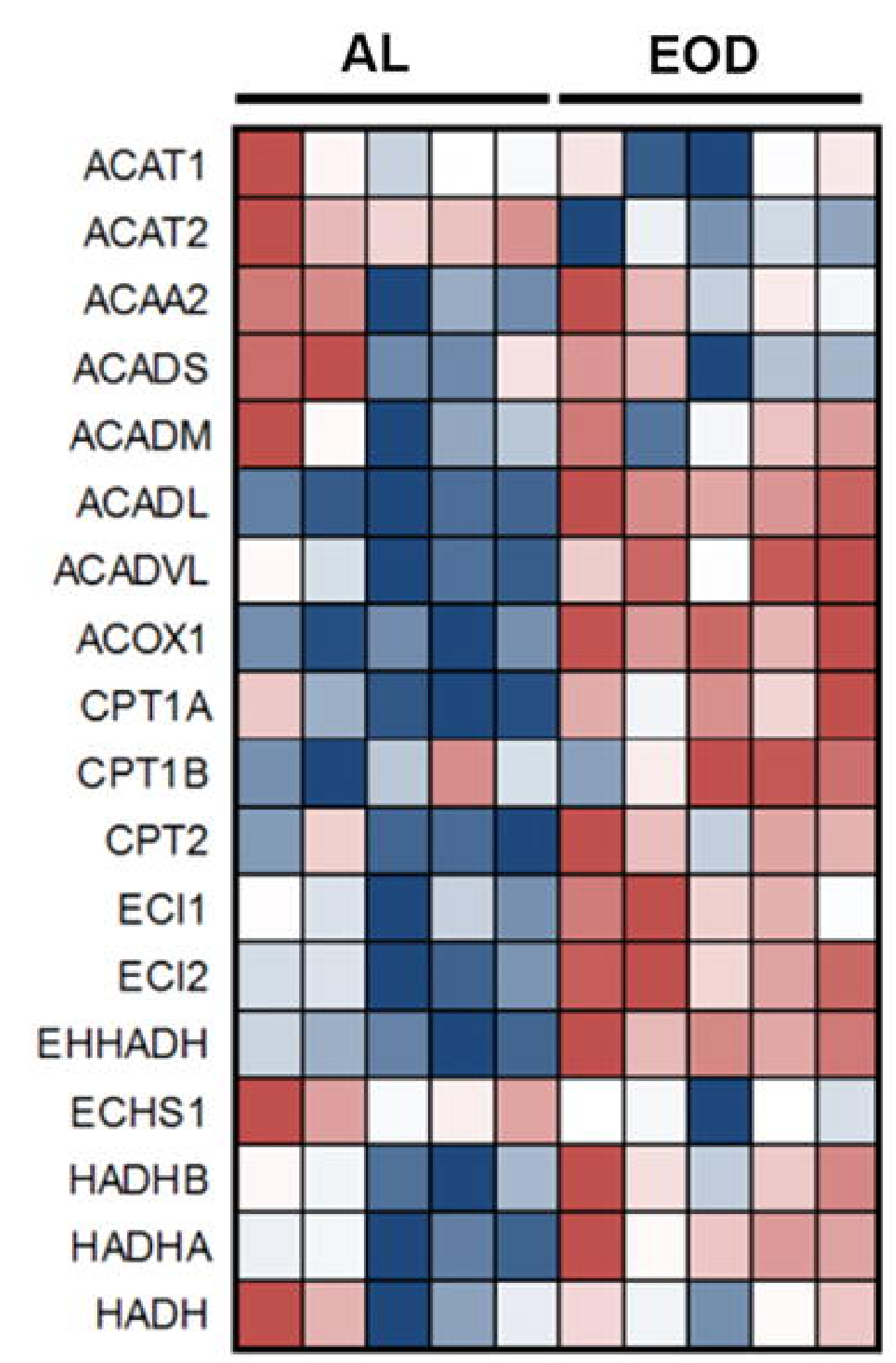

Transcriptome

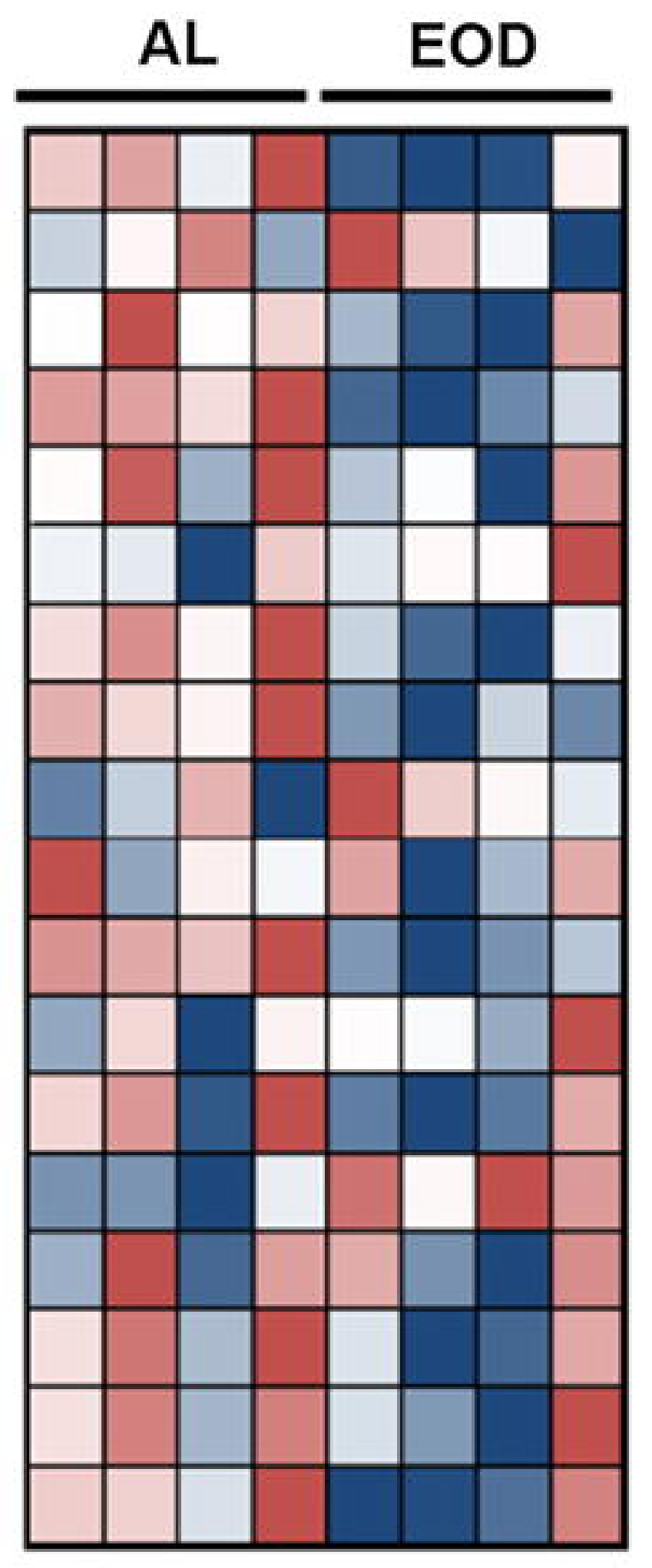

Proteome
Abundance (Log2)

Min $\quad$ Max

d

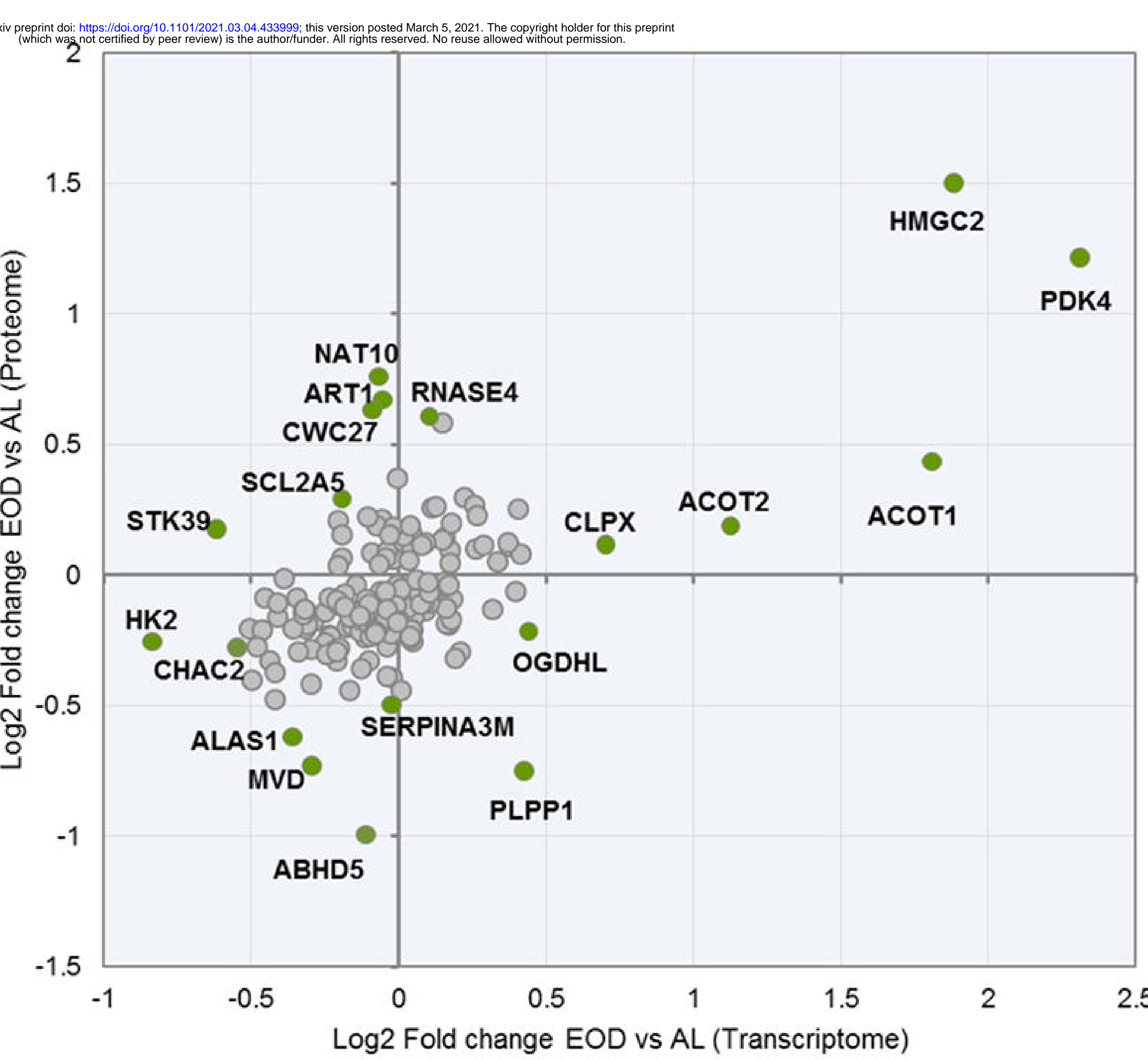

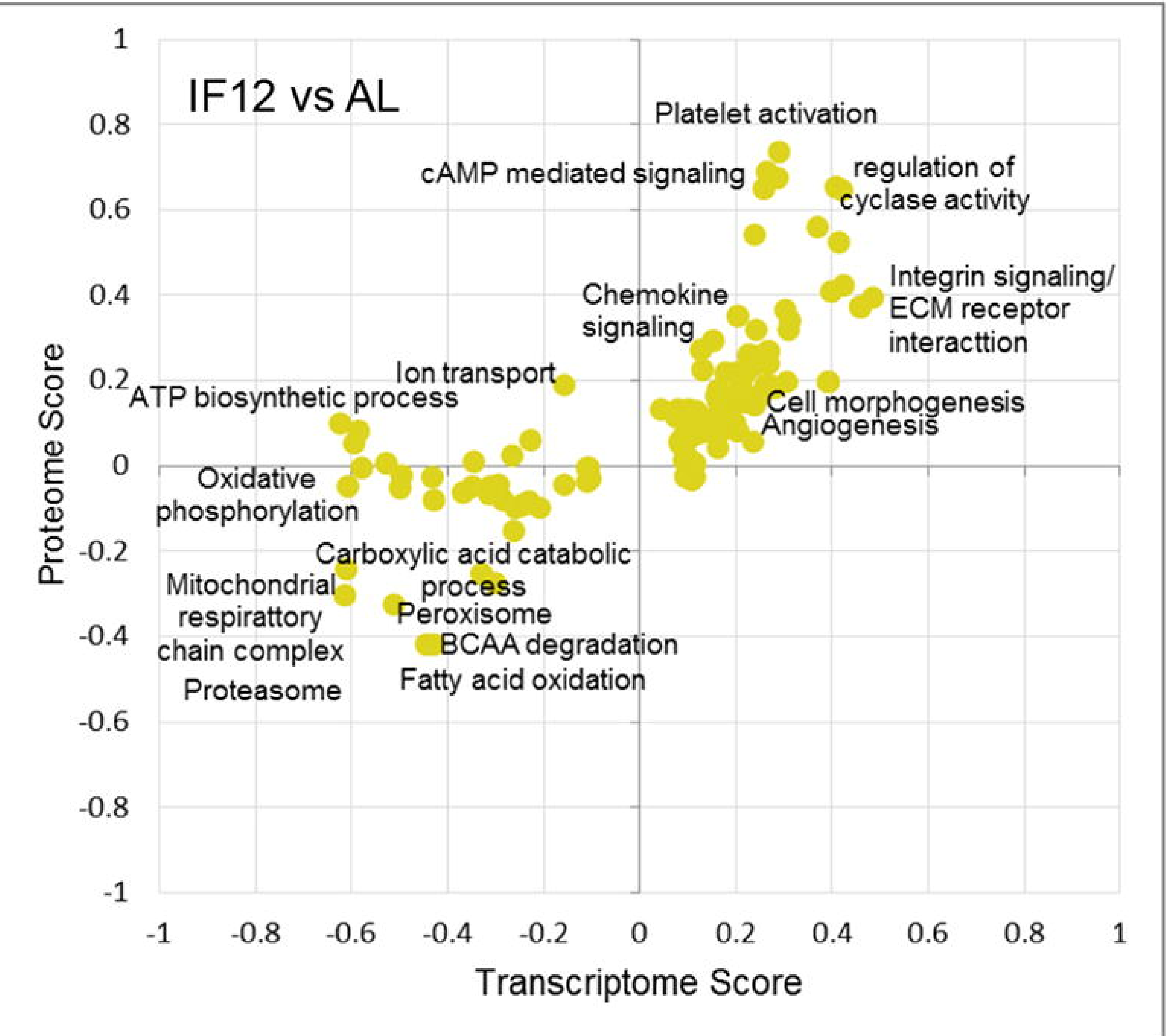
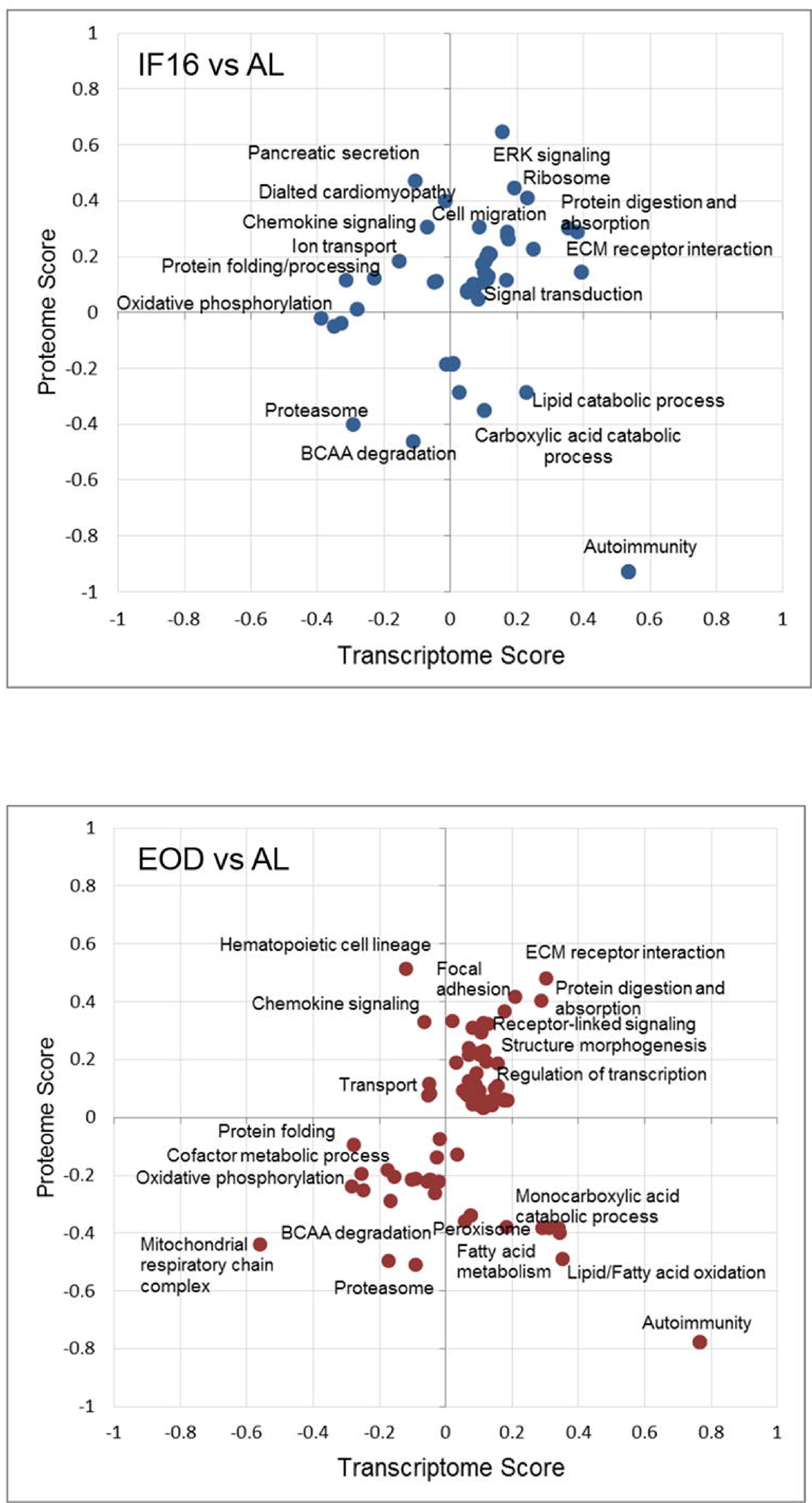

\section{Figure 3}


a

IF12 vs AL
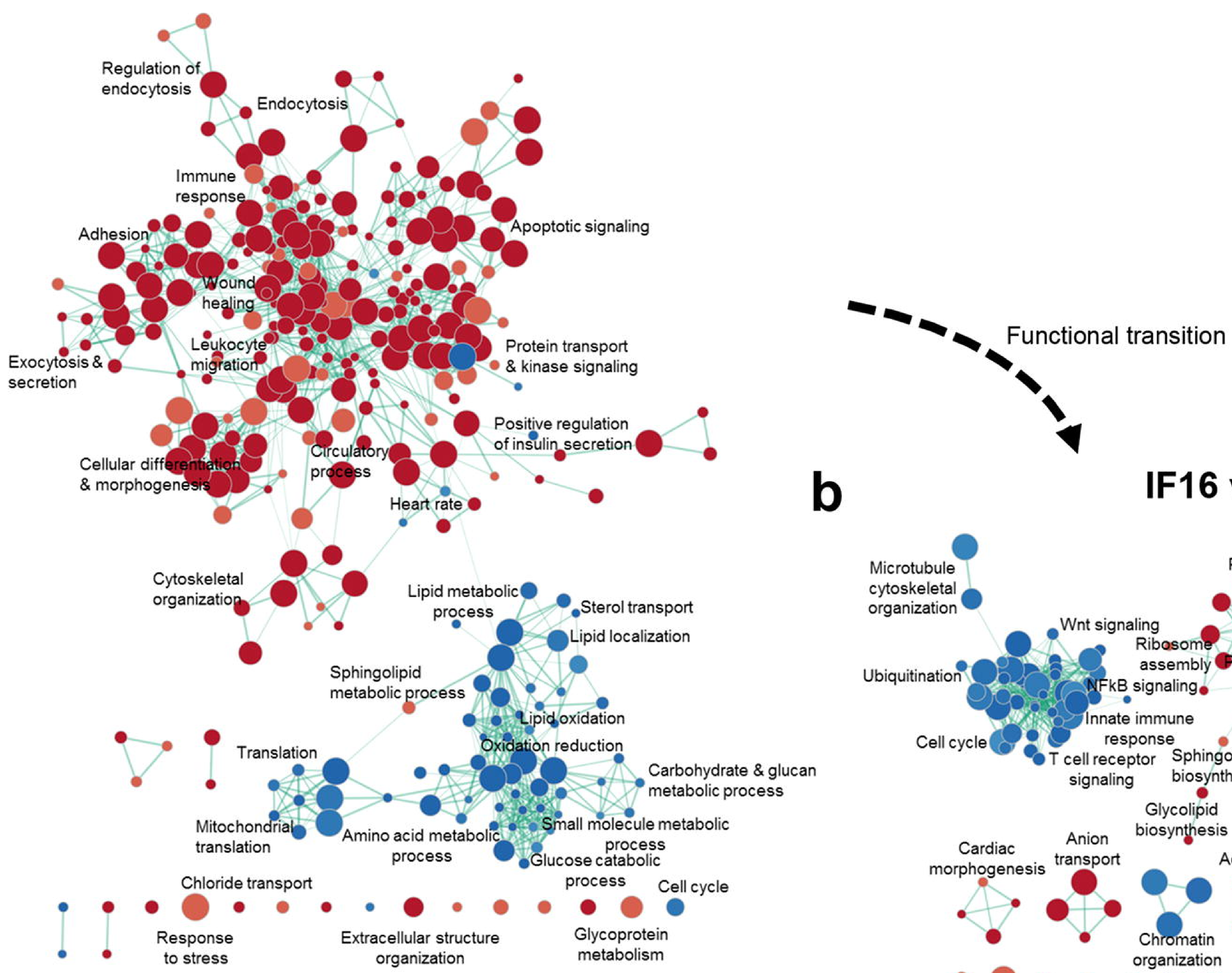

b

IF16 vs IF12

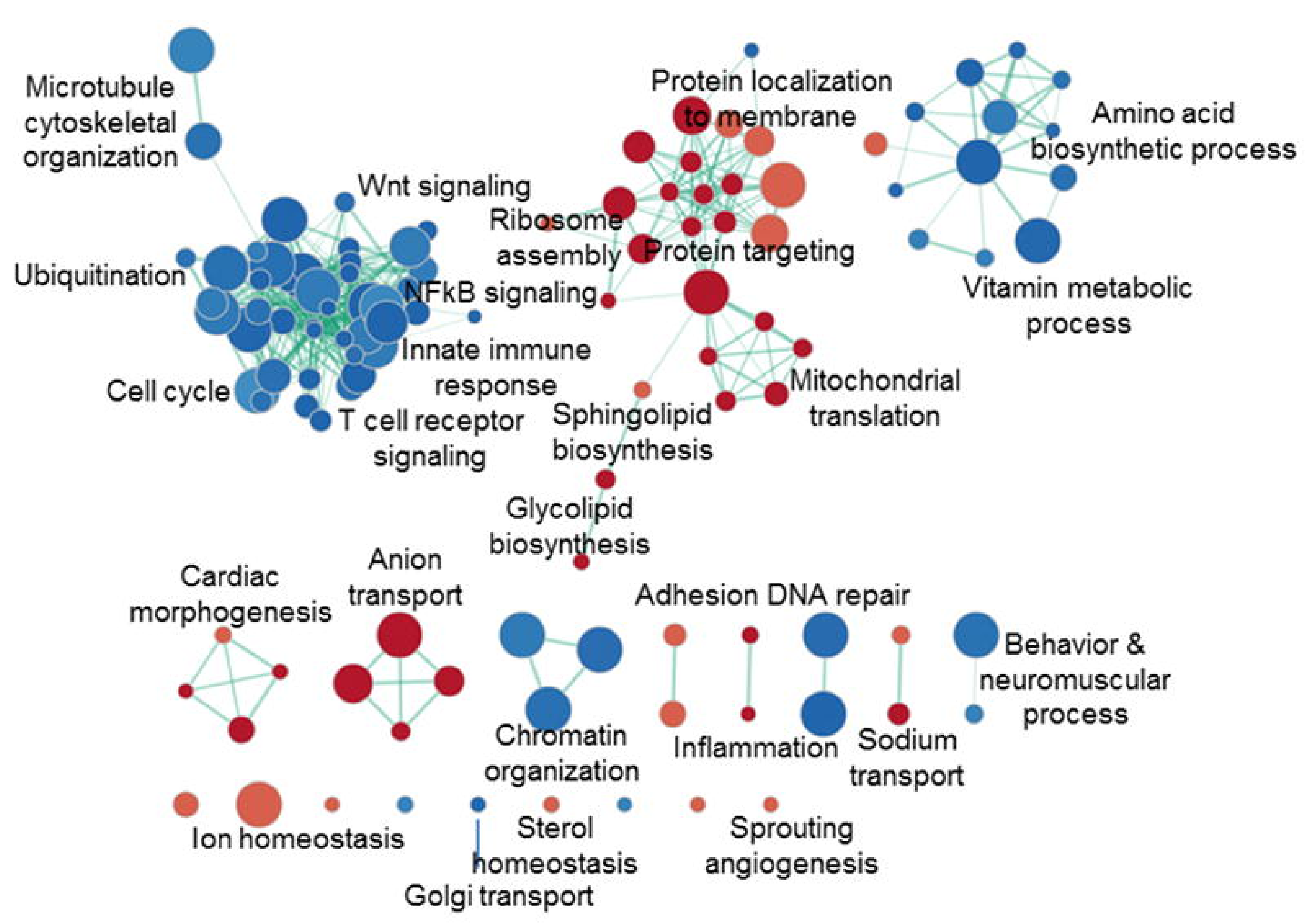

C

EOD vs IF16

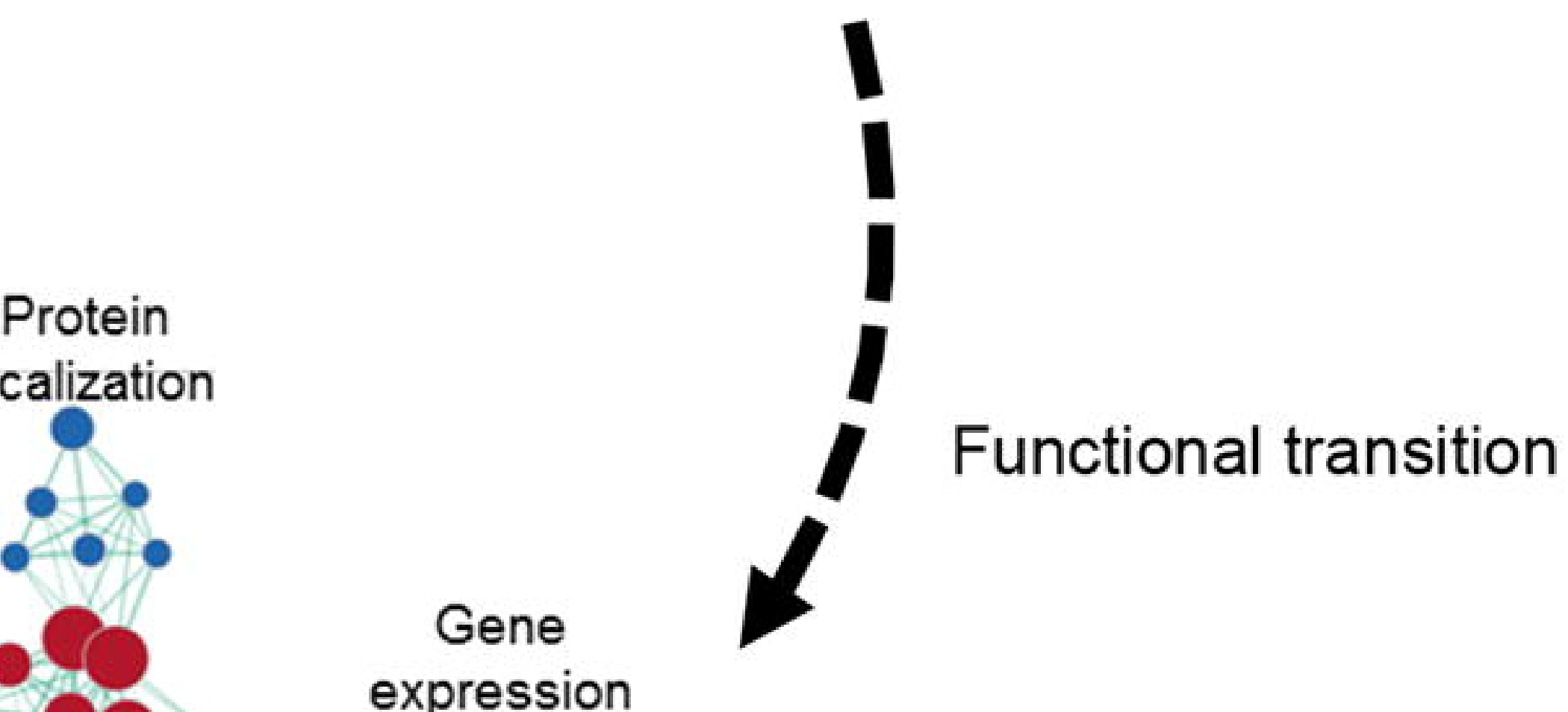

Figure 4

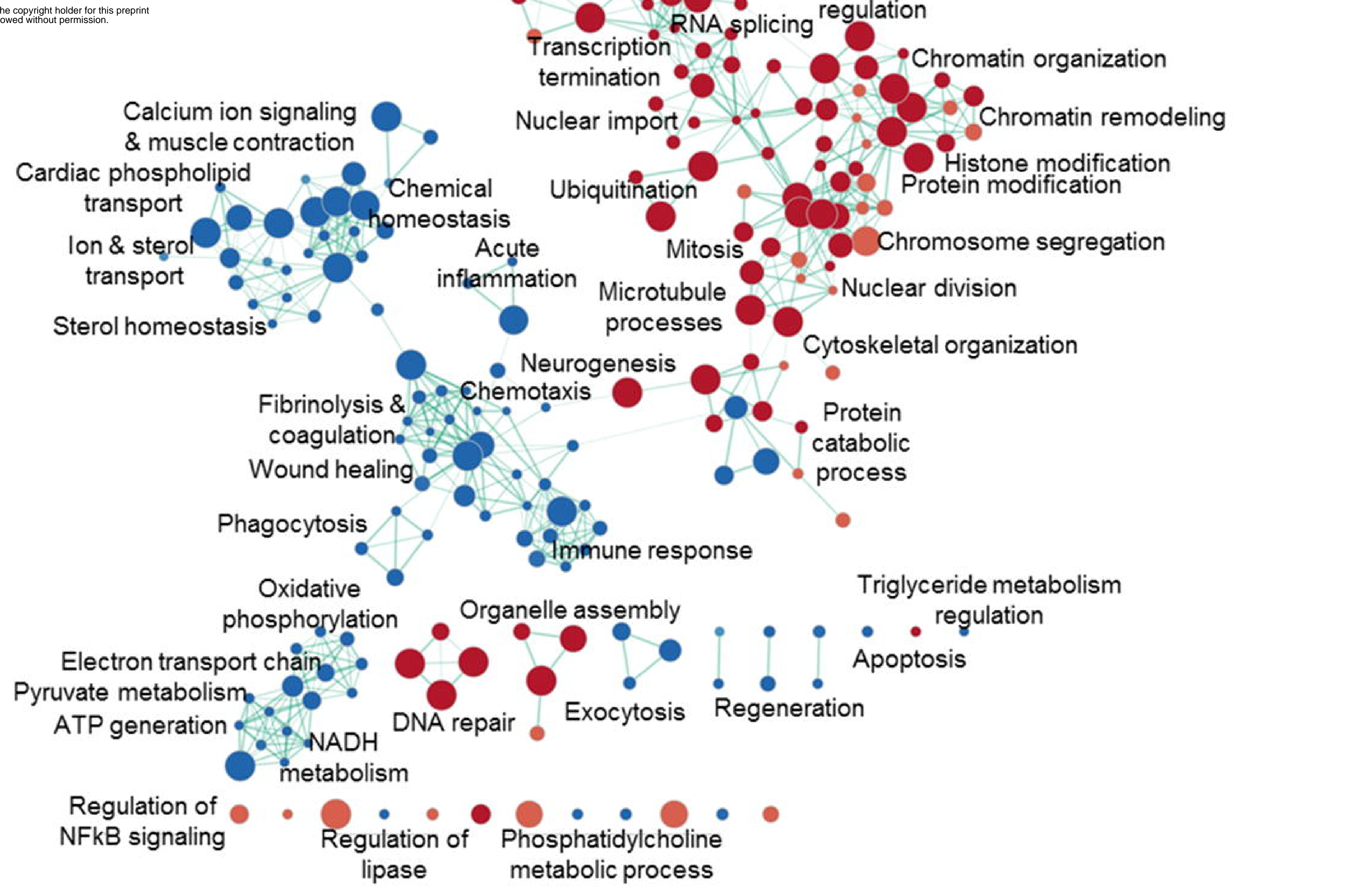


a
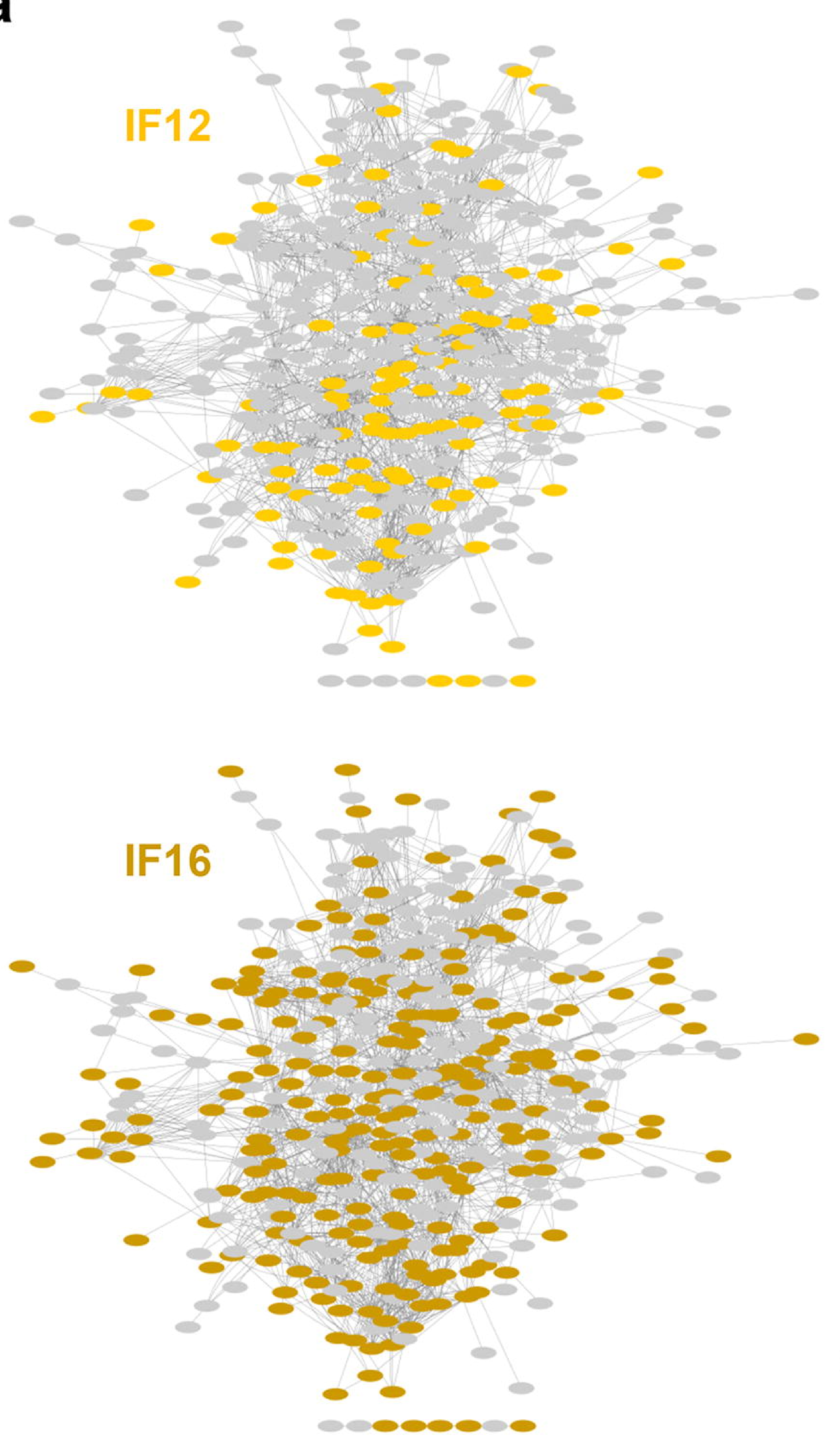

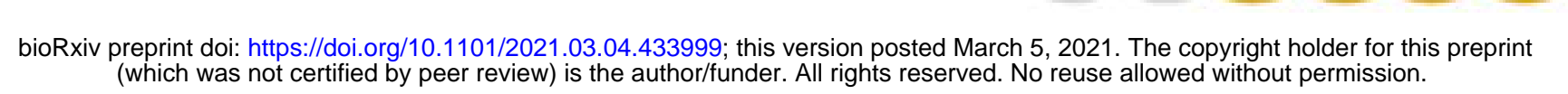

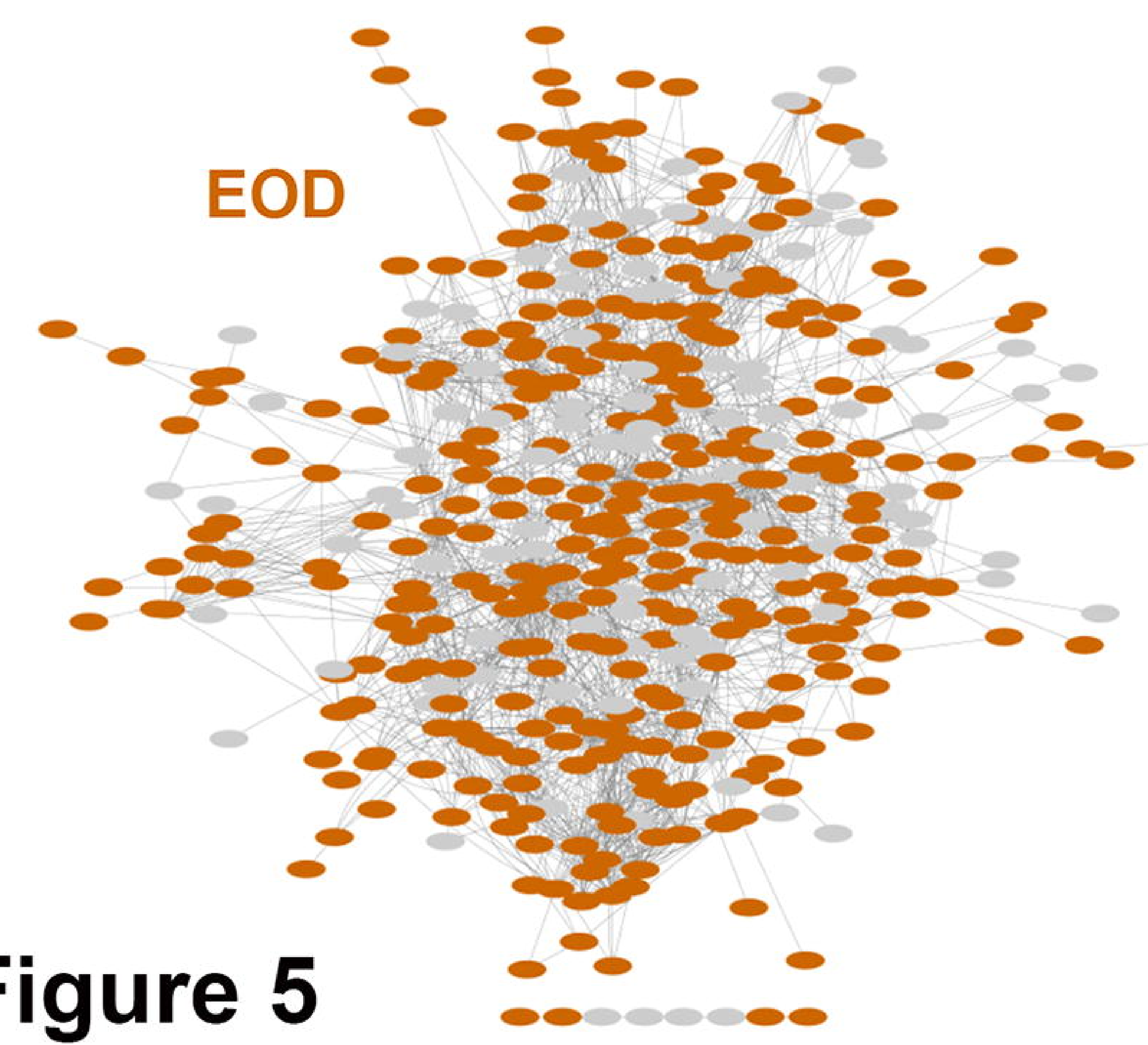

b

Fatty acid beta-oxidation \& BCAA catabolic process
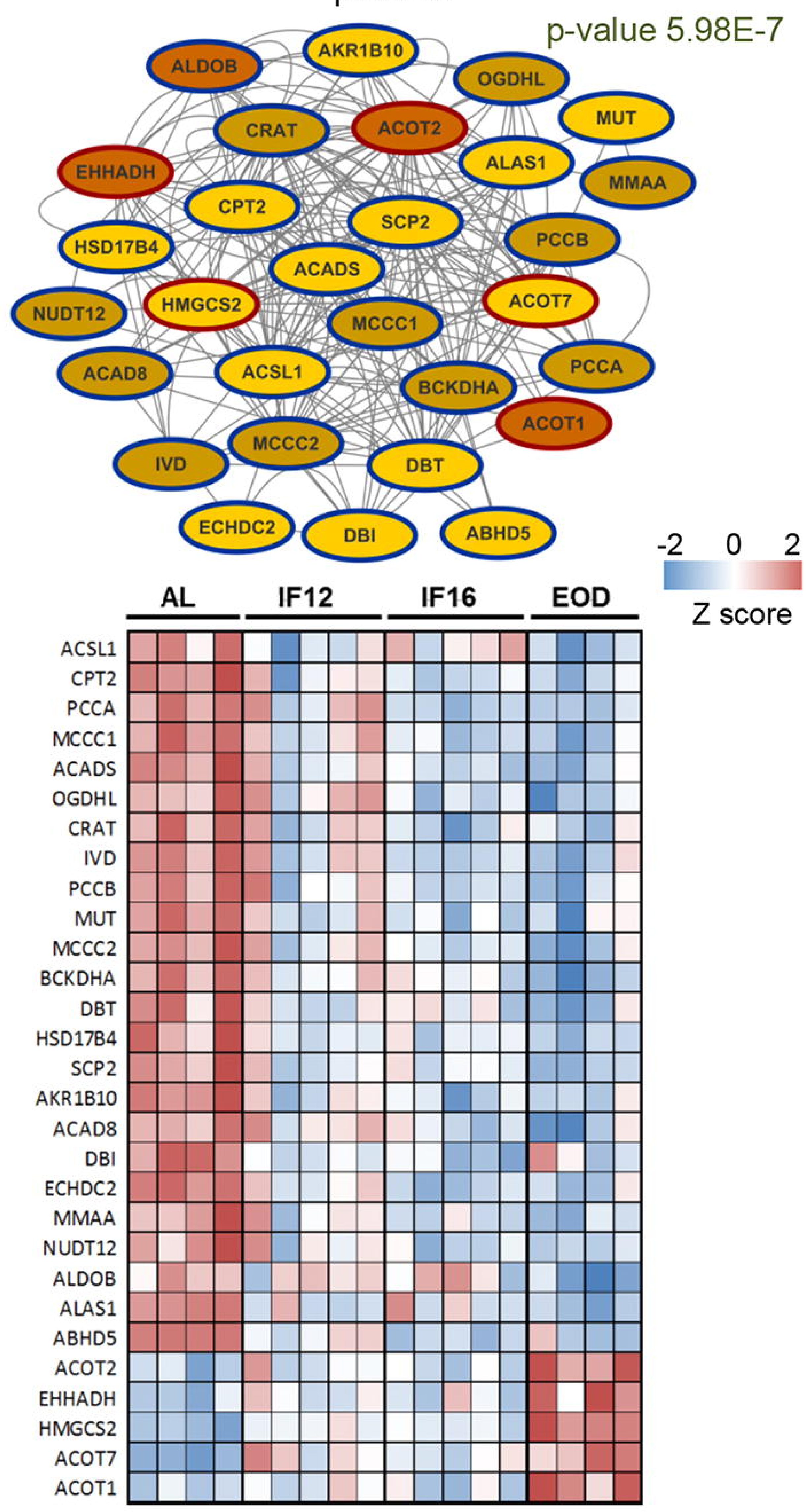

C

Cholesterol efflux, triglyceride homeostasis \& chylomicron clearance

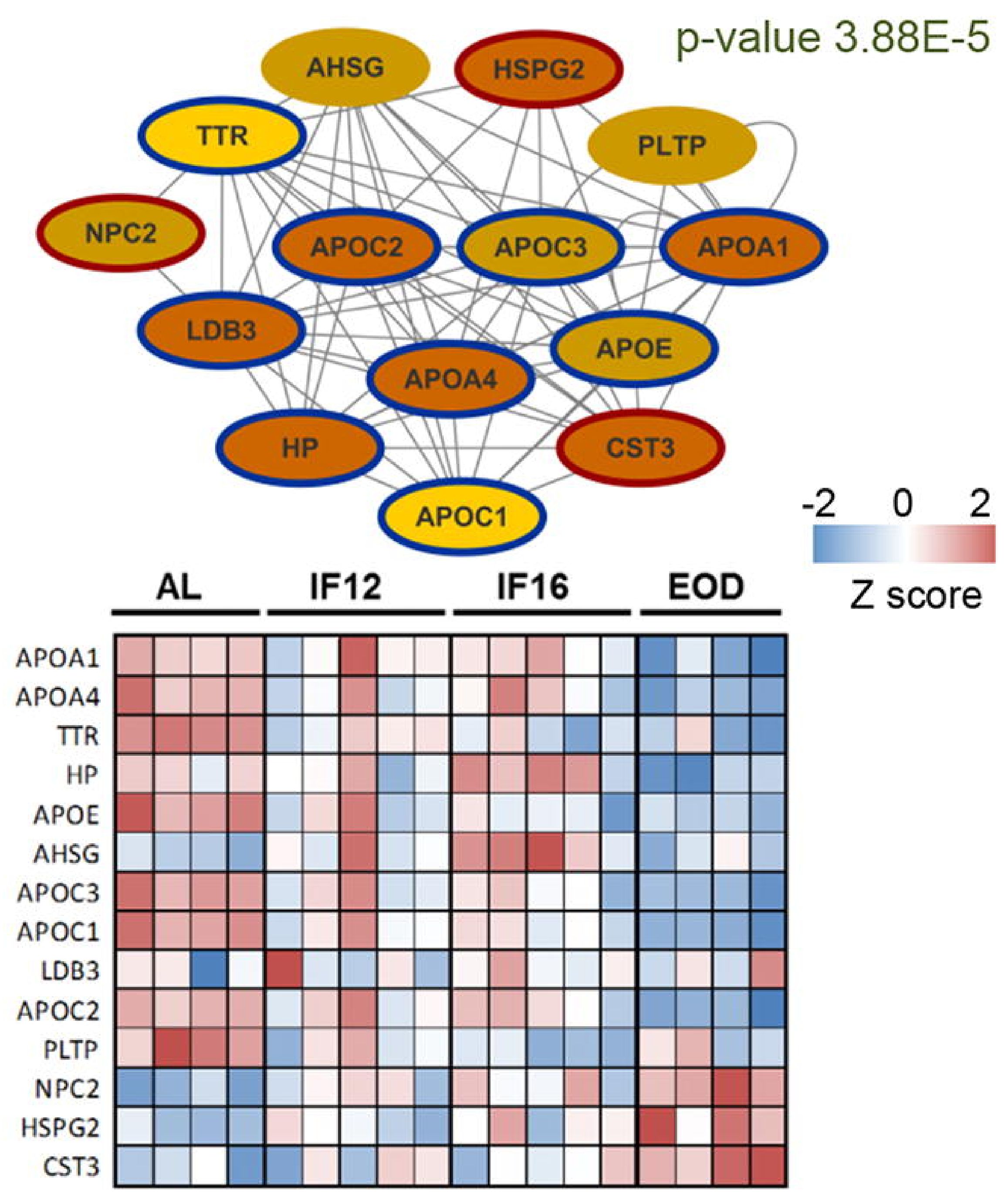



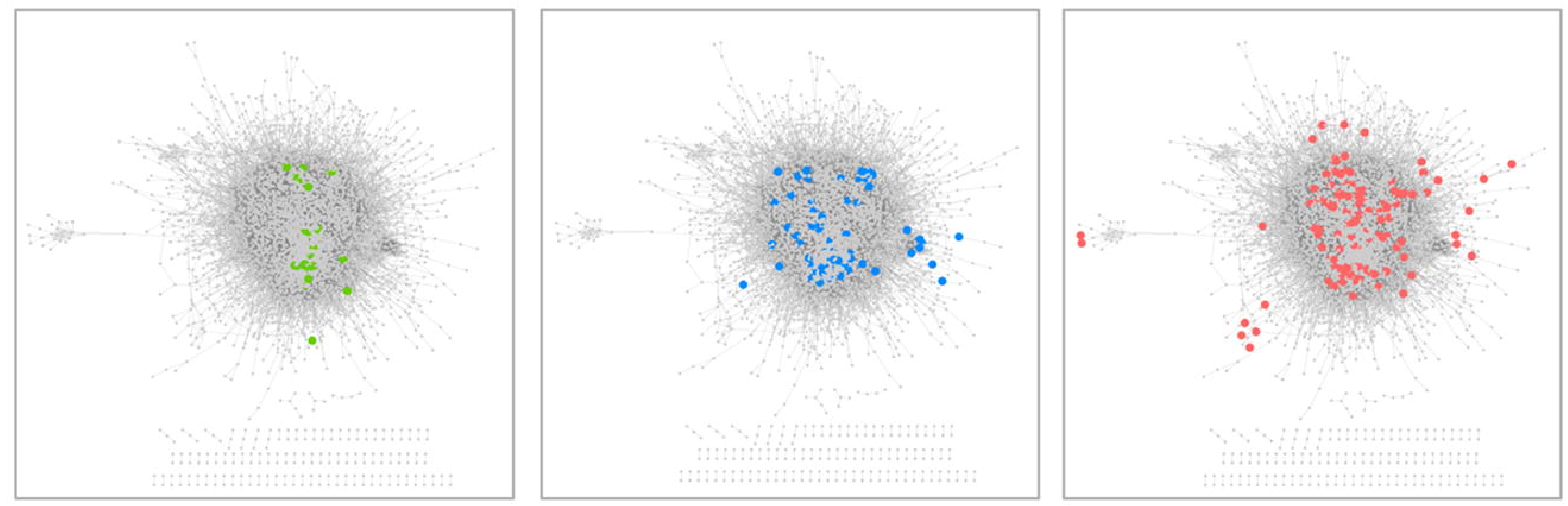

Carbohydrate metabolic process Oxidation reduction Glycogen metabolic process
Mitochondrion

Glutathione metabolic process b

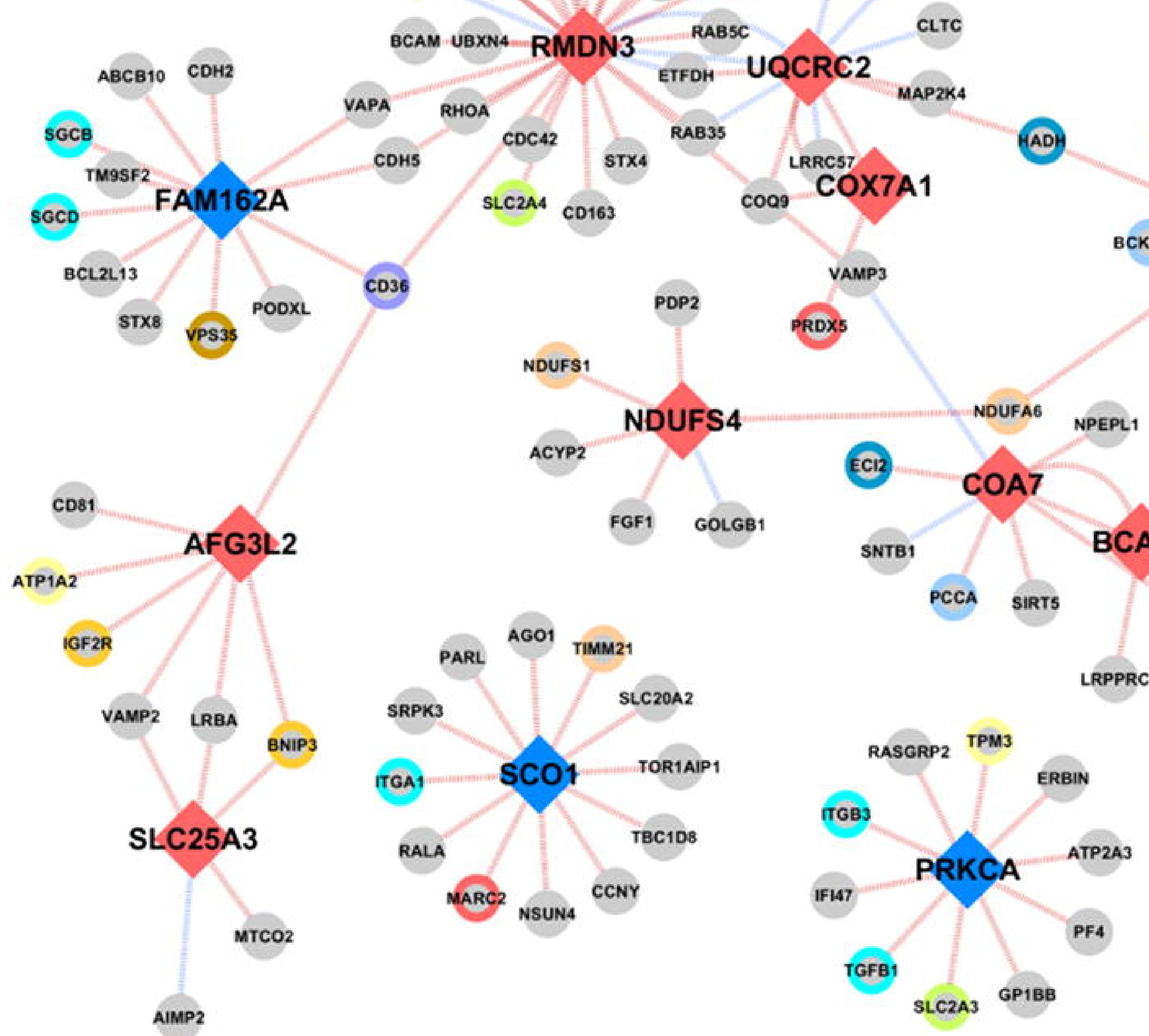

GNA11 RTN4 GANAB VTH1

ABCC8 ATPGVOA 1 GANAB RABGA TOMm70

LOHB VAPB CAVI RABGA H3F3C FGB

FERMT2 ${ }_{\text {KPNB1 }}$
Patelet activation

Fatty acid oxidation

Migration

Translation
Translation mRNA transport

PPAR signaling

Lipid binding \& efflux

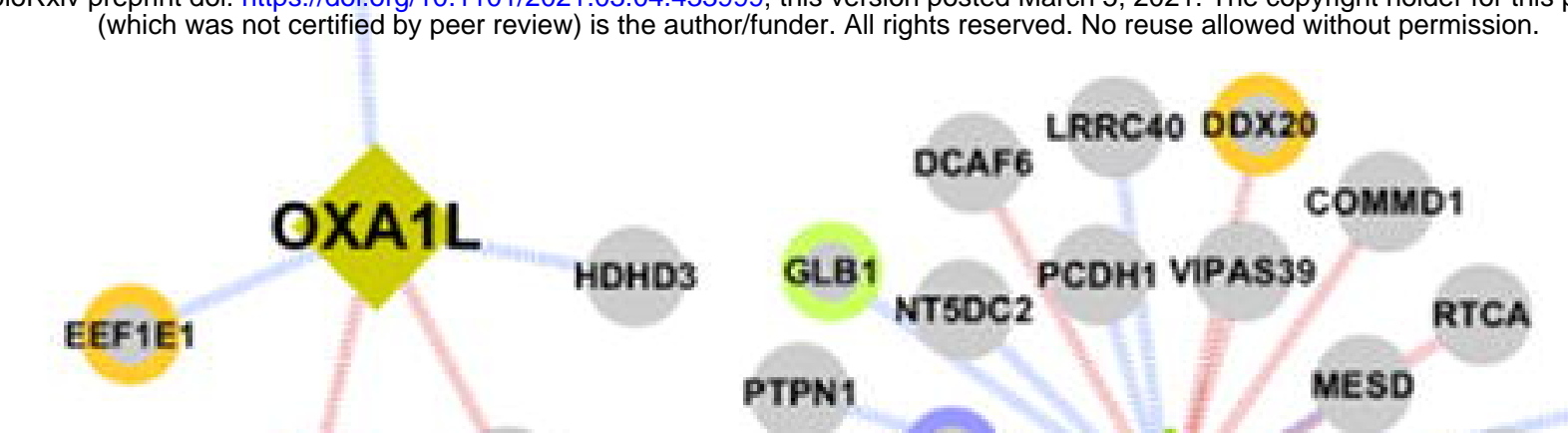

(อ11. SLC12A4

SNRPC EIFAEBP1 MGME1 TKFC CLASP TMEM161A LSMG CUL ISOC2B PHAX SPRYD7 BNIP2 RALGAPA1 ANAPC1 OOF2 ${ }^{\text {RLIM }}$

MRP

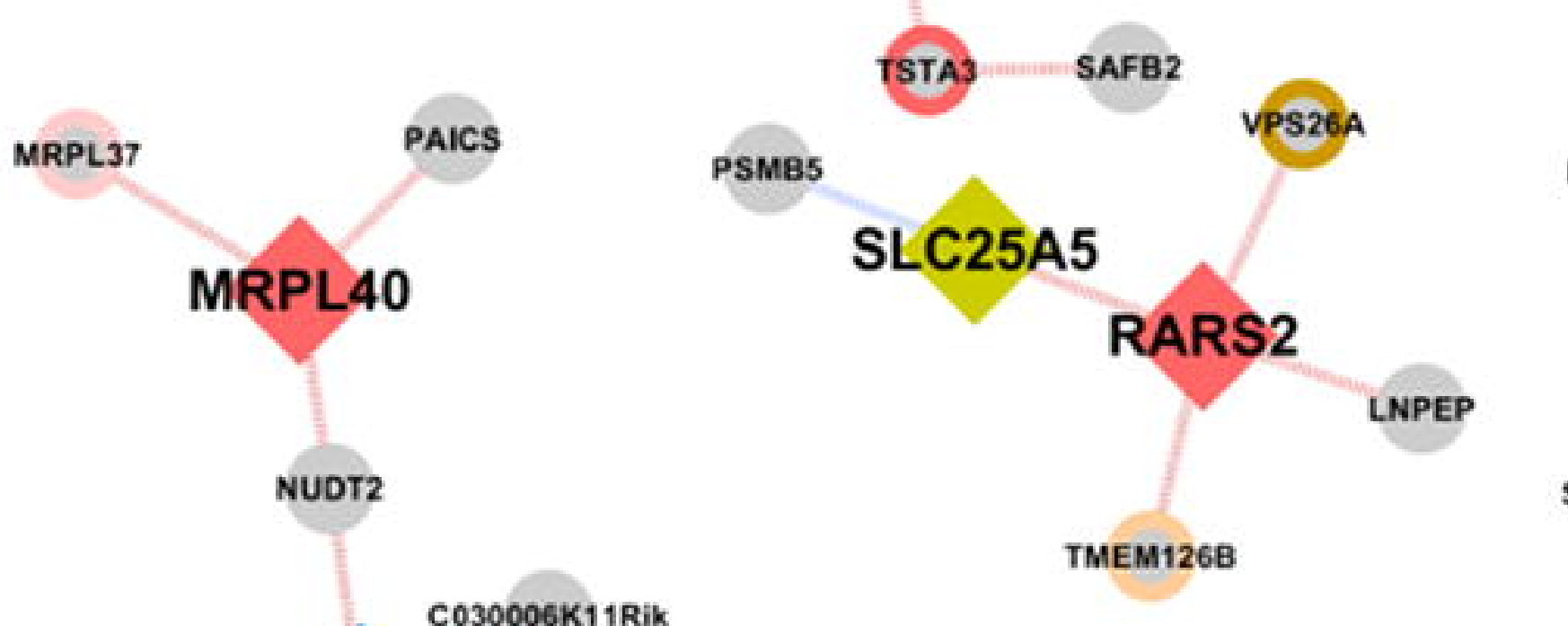

NUD

MLYCD

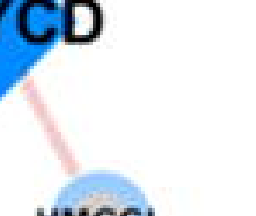
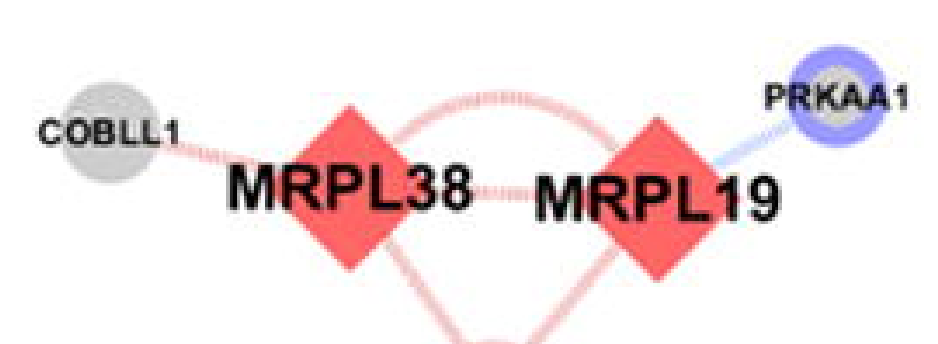

PHB NDUFAF1 MRPS16

ACOT13

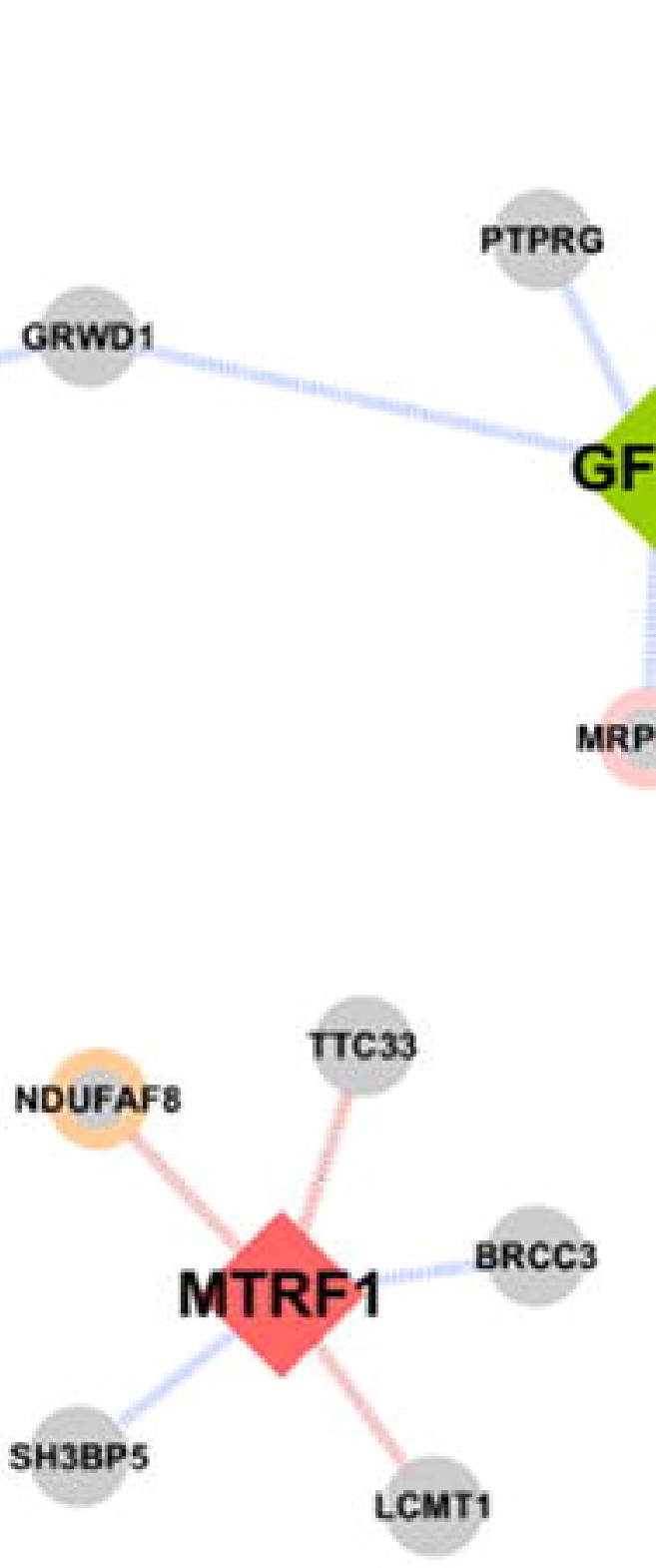

\author{
(xiv) EeFriar

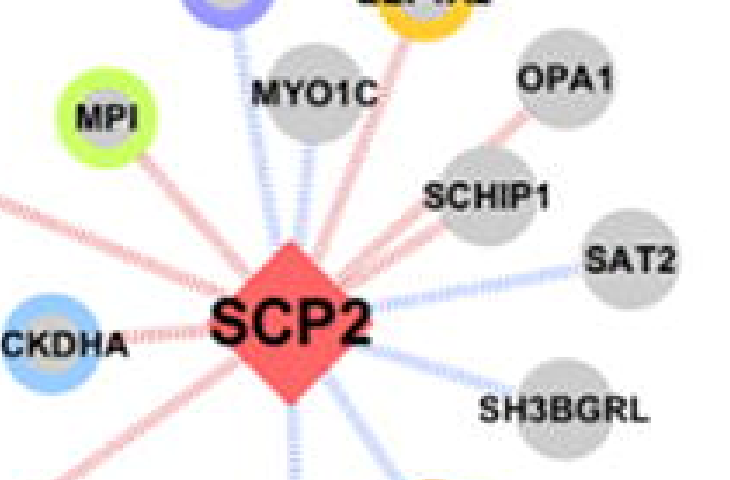

\section{ग्खिक्ष}<smiles>[C]1CCCCC1</smiles>

(aㅐ<smiles>CCCC1CCCCC12CCCC2</smiles>

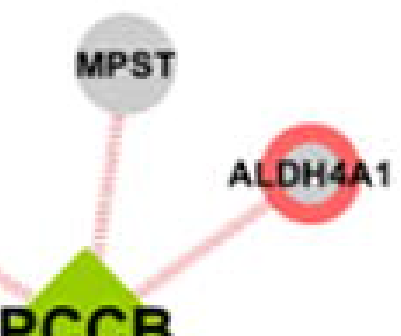

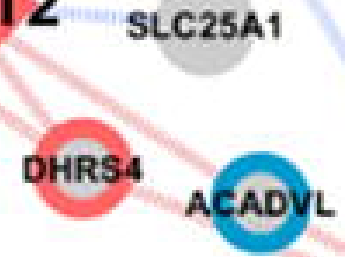<smiles>O=C(O)C1CCCCC1</smiles><smiles>C1C[GeH]2C3CCC3C2[GeH]1</smiles>
vockn
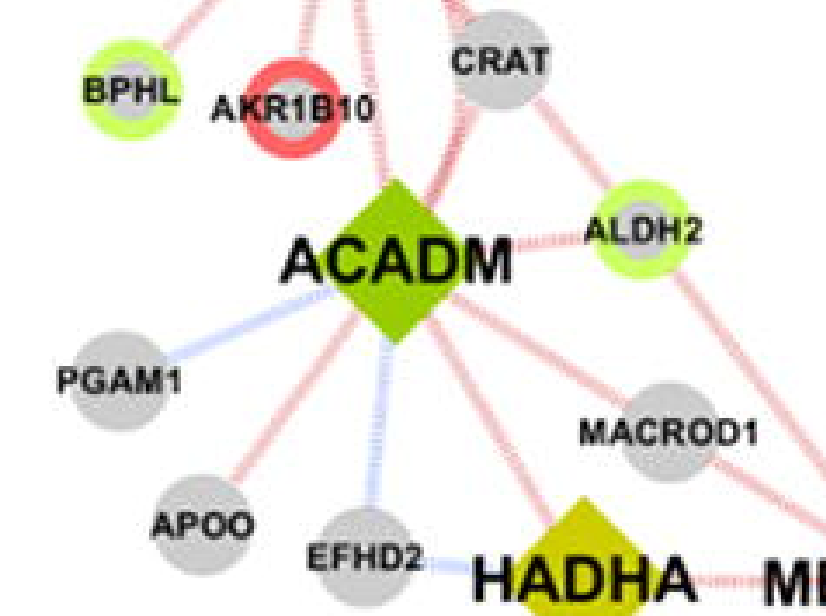

ॠ

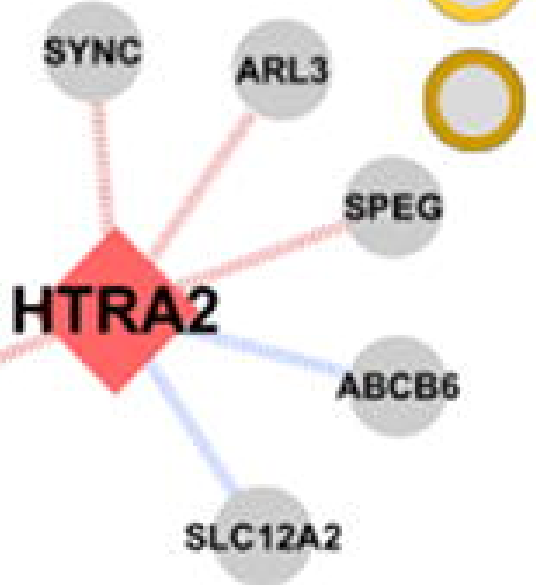

AMPK signaling

Branched chain catabolic process

Carbohydrate metabolism

Cardiac muscle contraction

Fatty acid beta oxidation

Hypertrophic cardiomyopathy

Mitochondrial respiratory chain complex assembly Mitochondrial translational elongation

Oxidation reduction

Oxidative phosphorylation

PPAR signaling

Regulation of apoptosis

Regulation of macroautophagy 


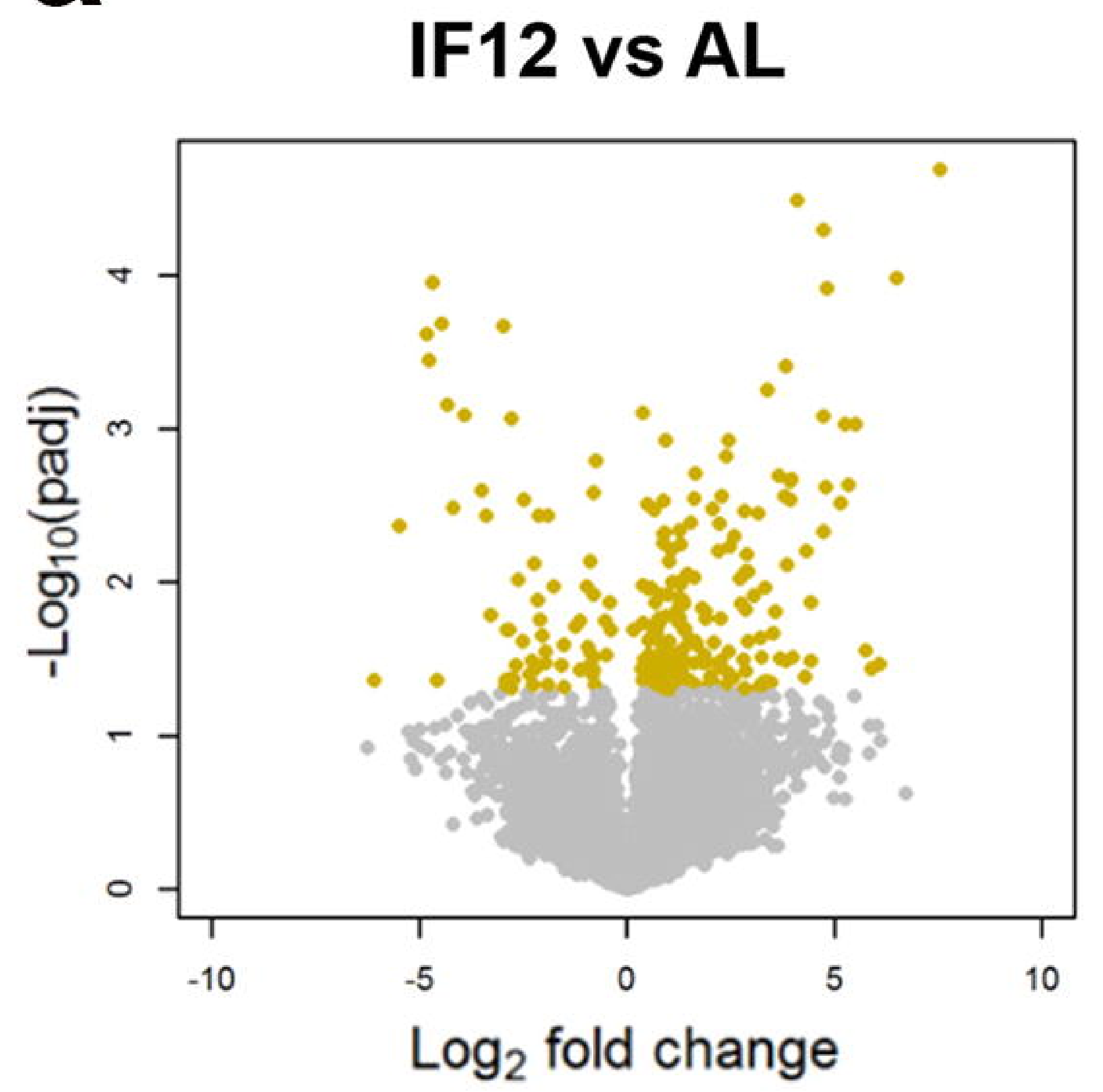

IF16 vs AL
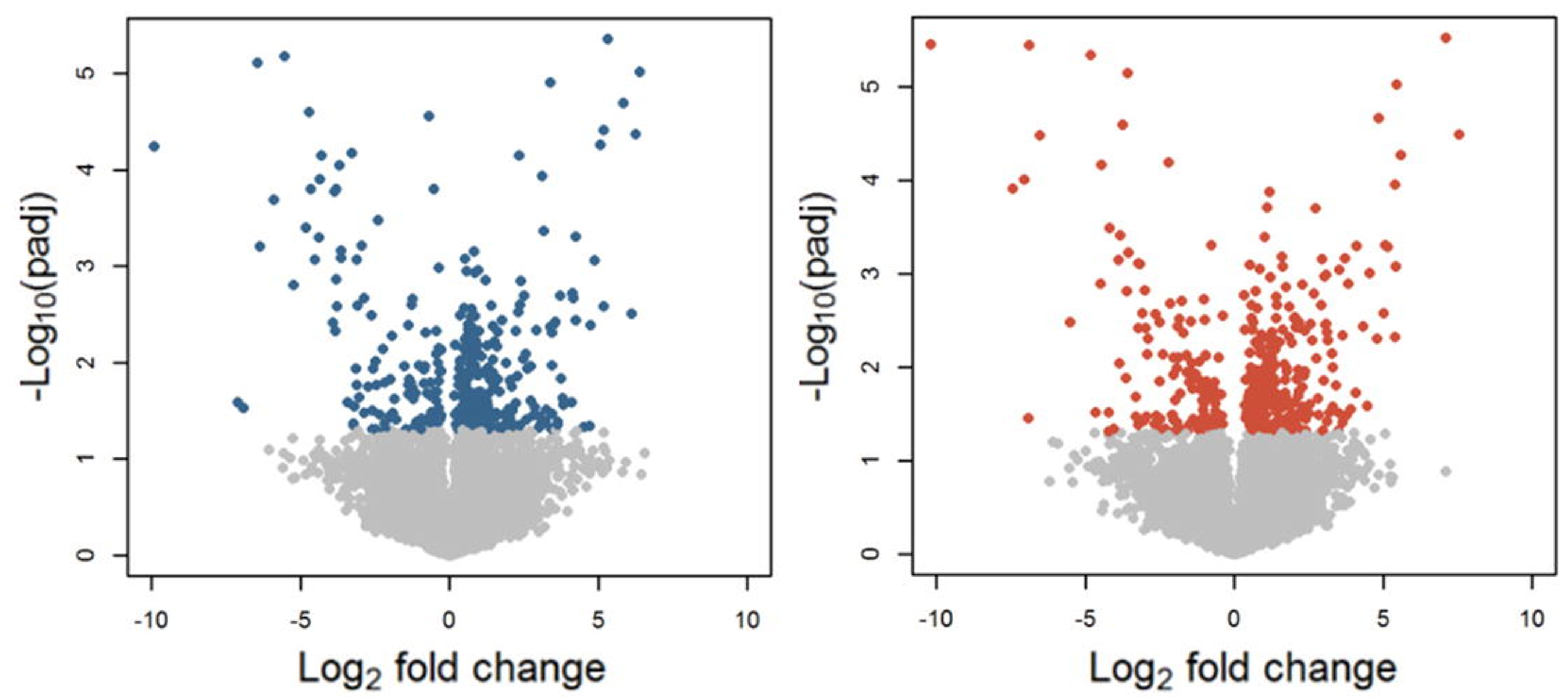

b

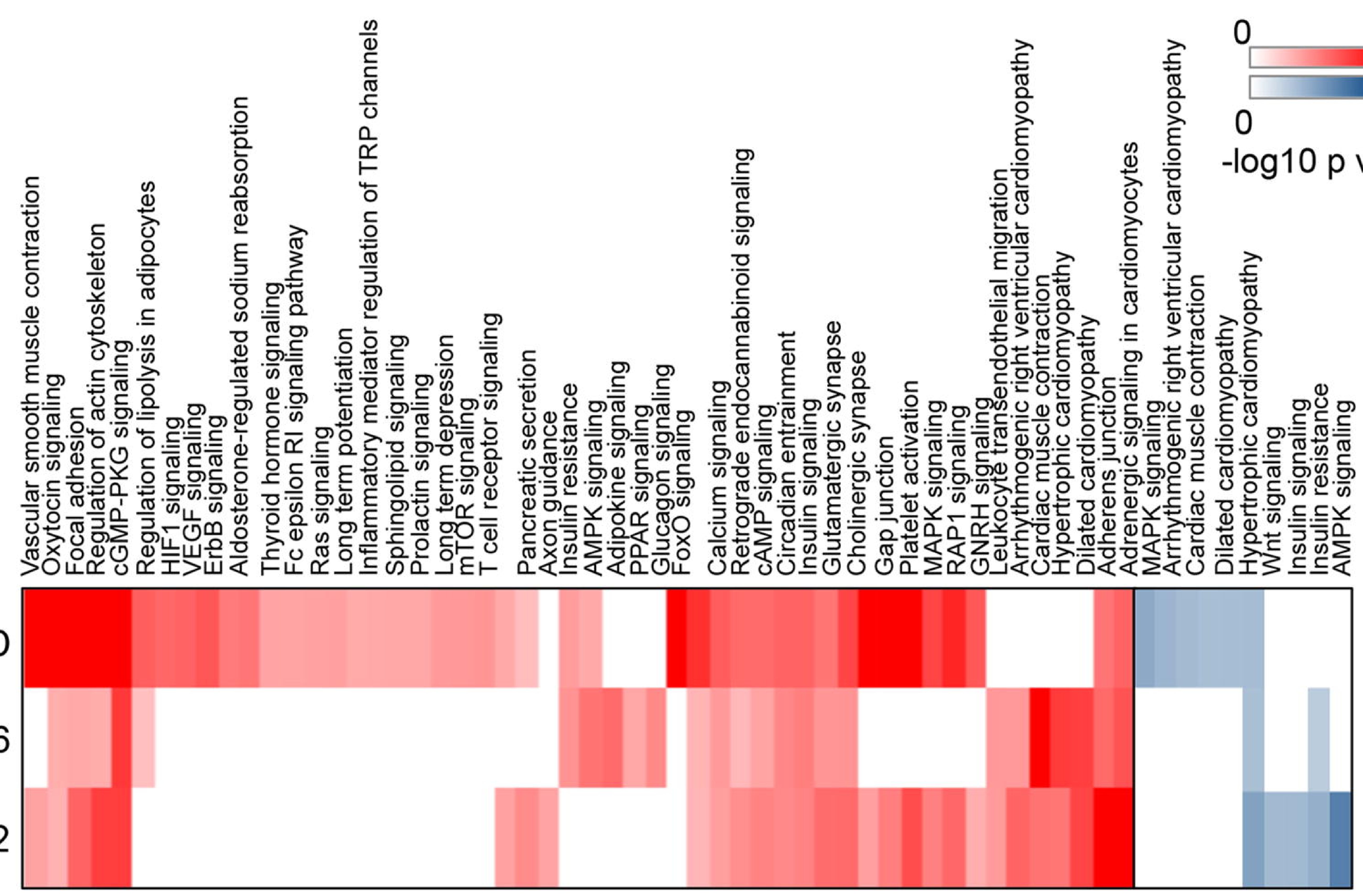

C
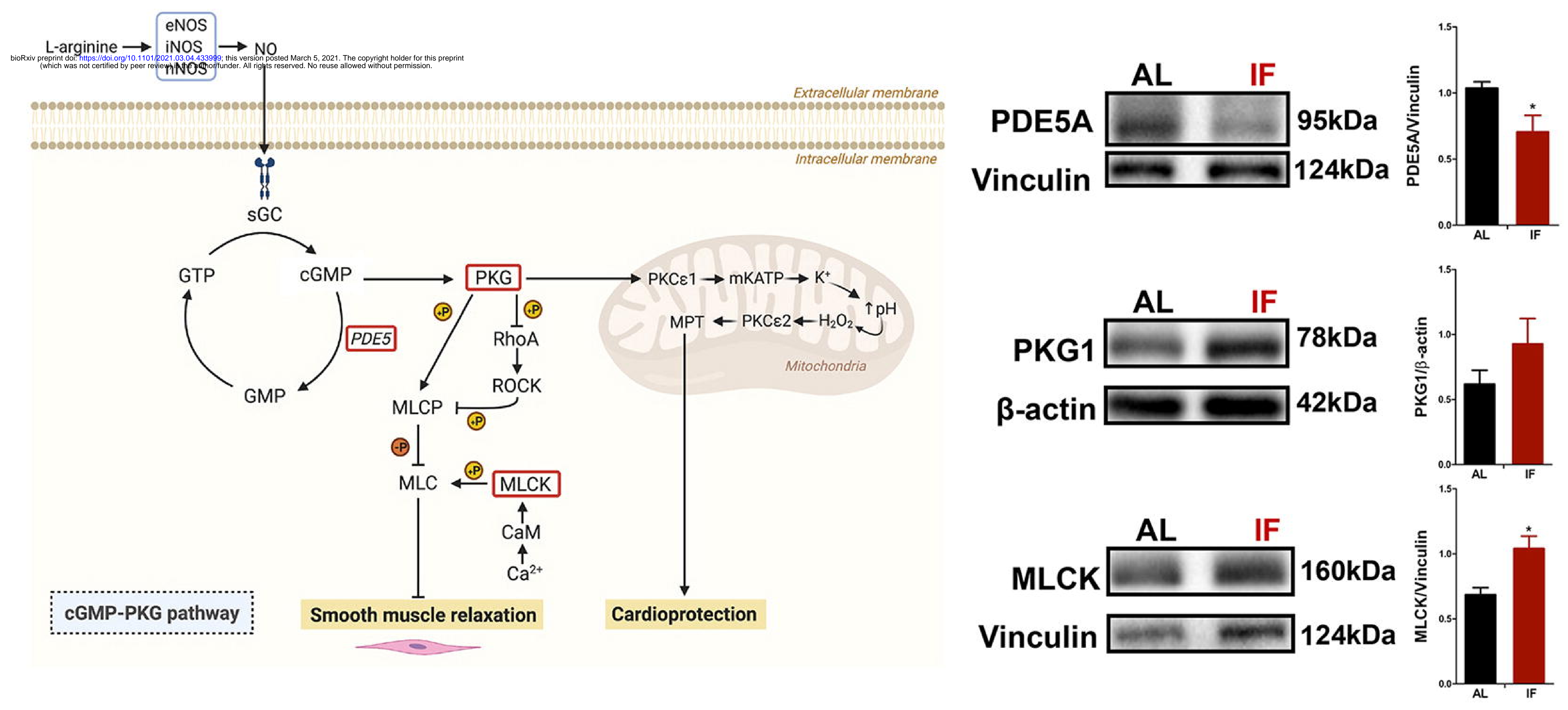

Figure 7 


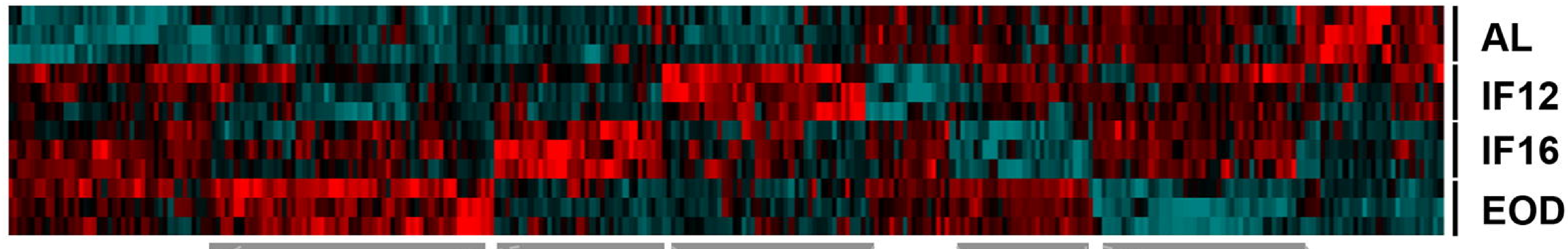

PKA motif

PKC/ PKC epsilon motif

CAMK2 motif

AMPK motif

$B$-adrenergic receptor motif AKT motif

\section{PLK1 PBD binding motif}

AMPK motif

CAMK1/4 motif

CSNK1 motif

b
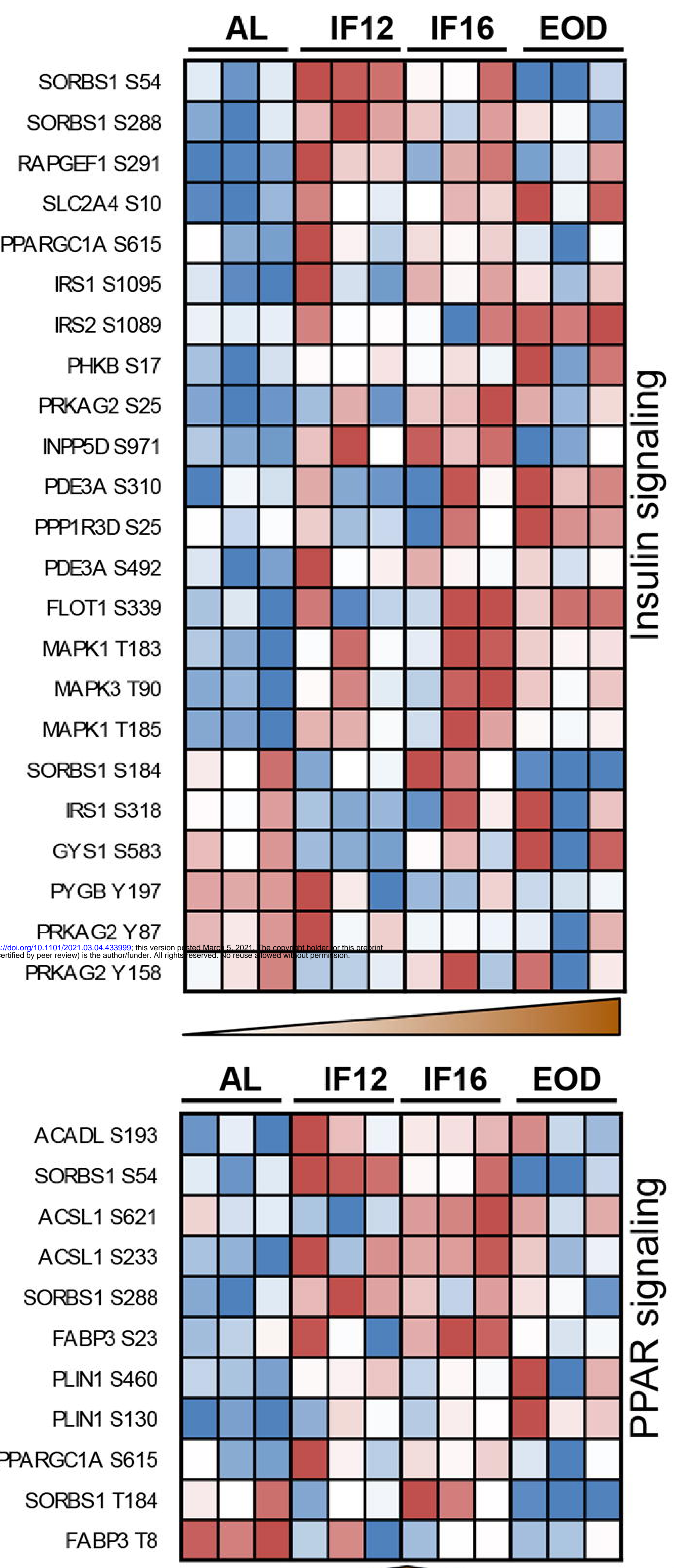

Figure 8 
$a$
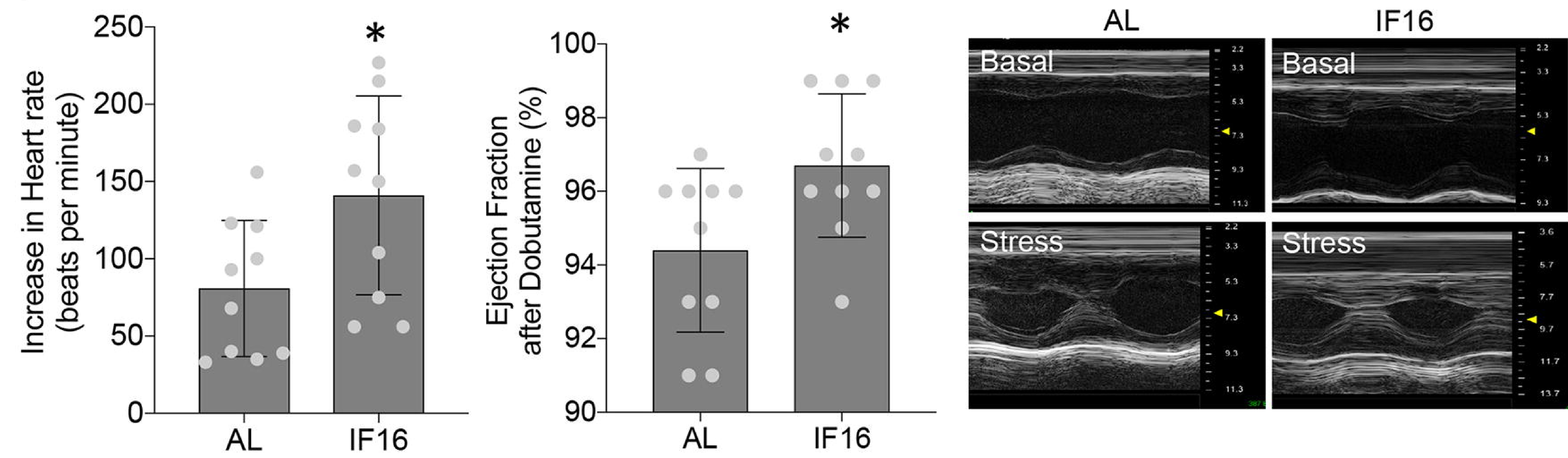

b
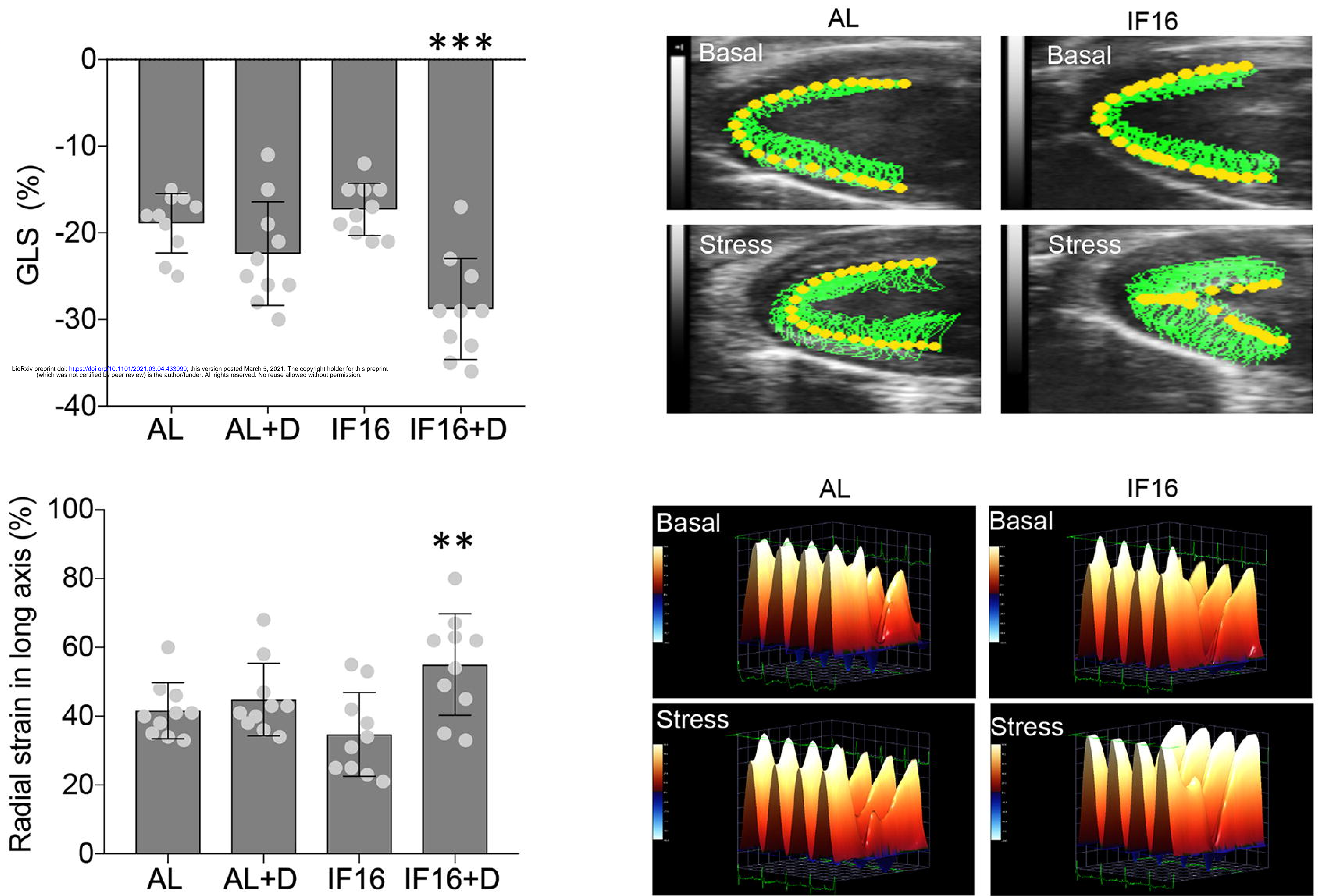

AL
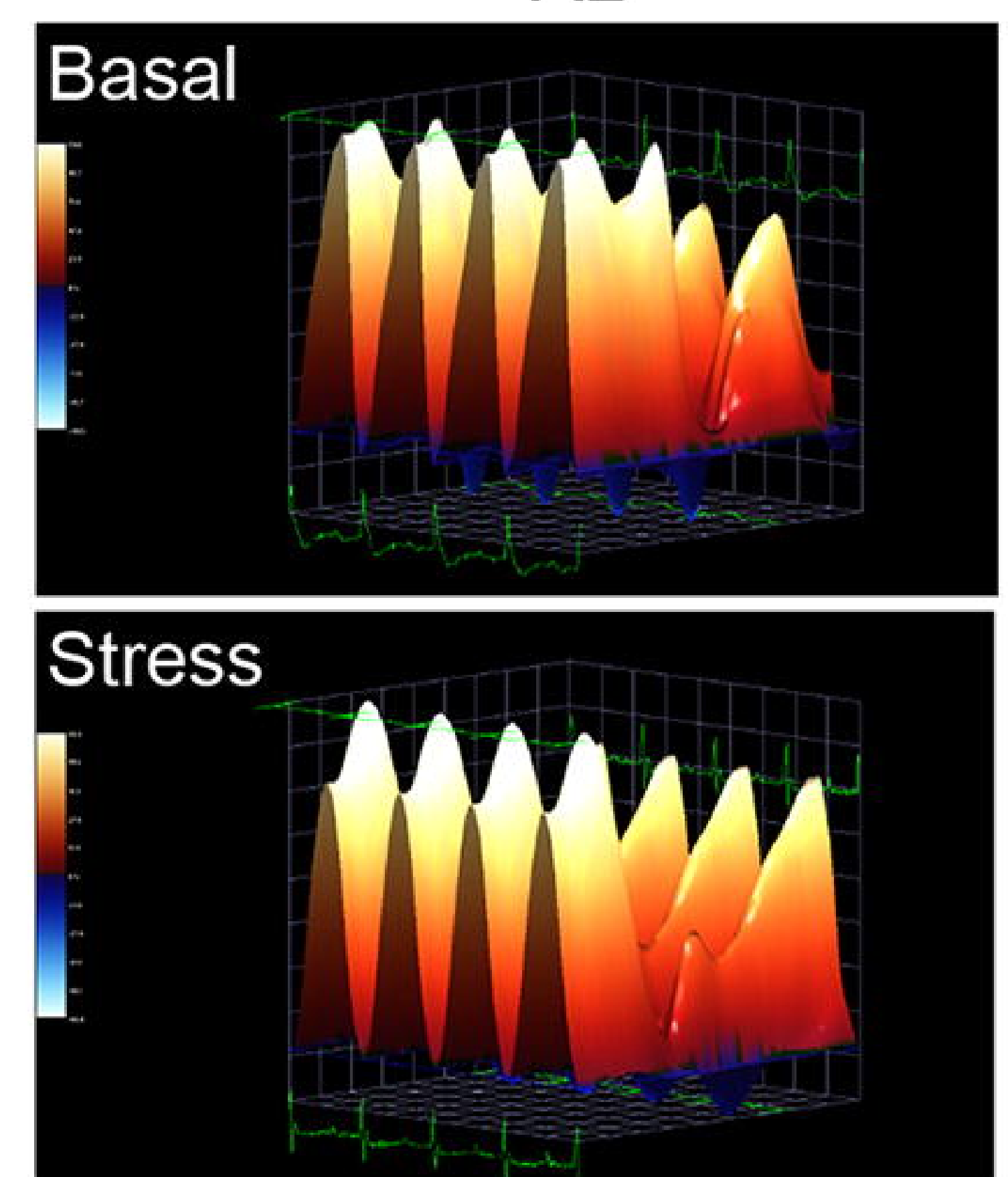

IF16
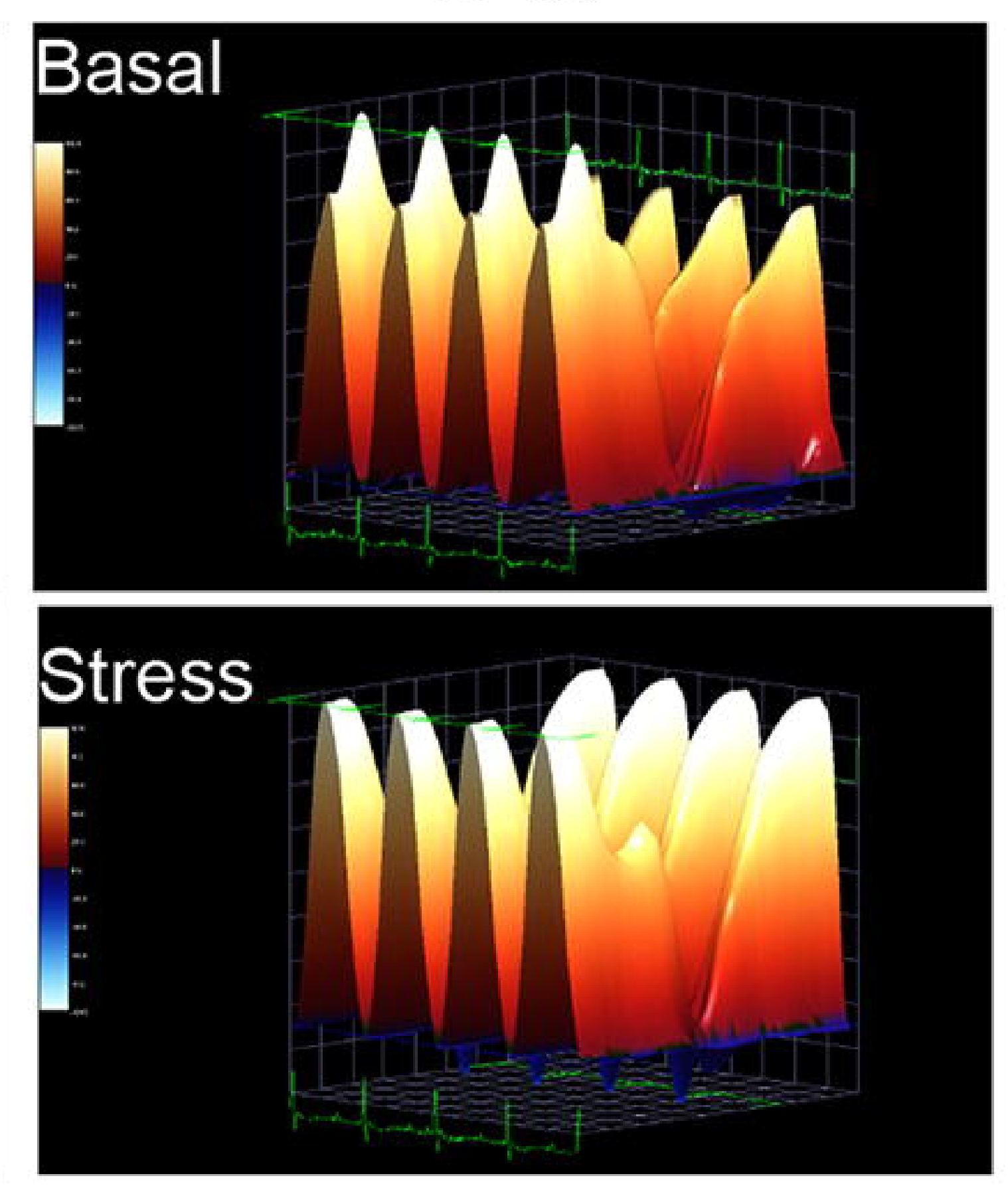

Figure 9 


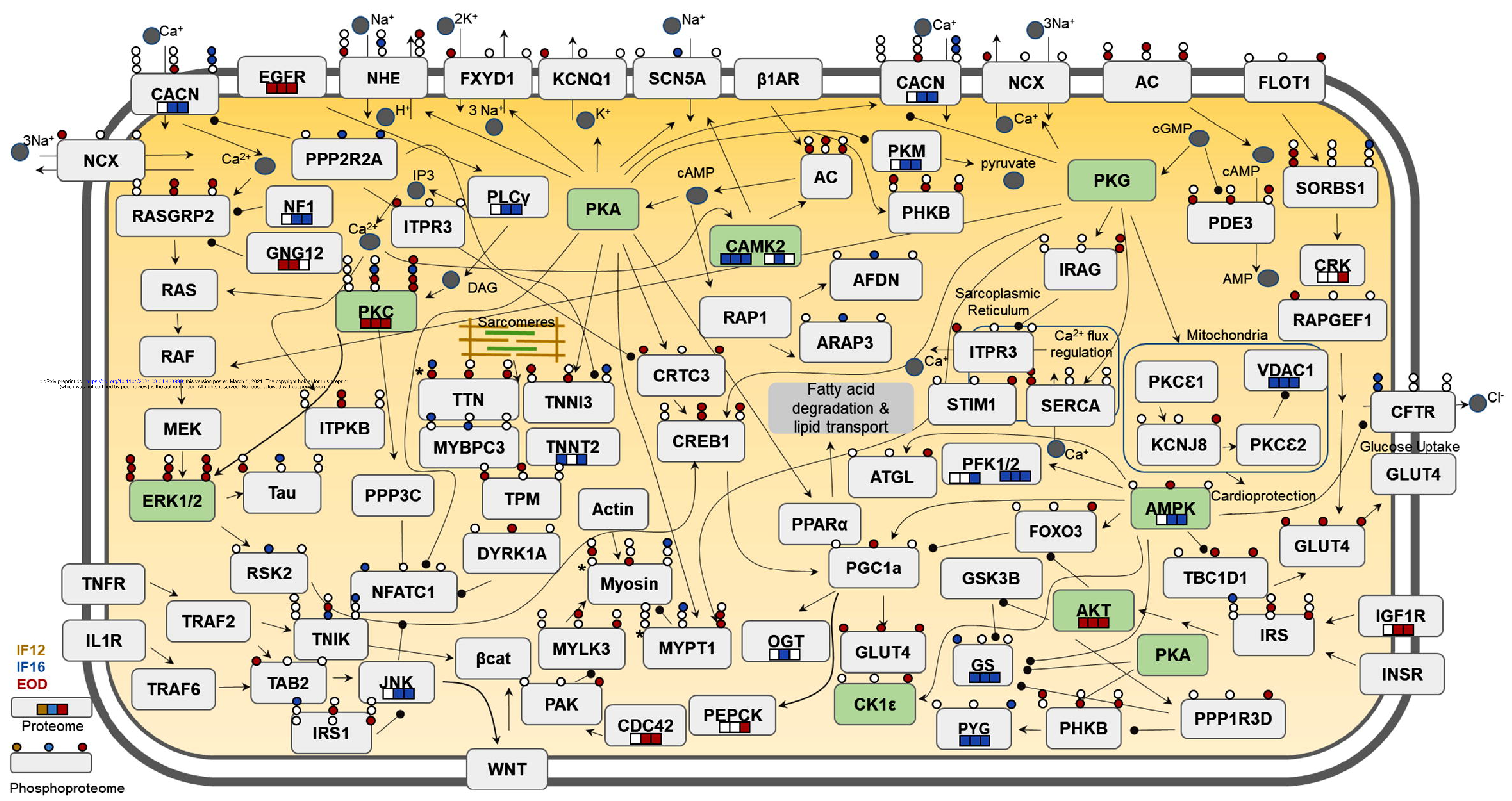

Figure 10 